IA WPE NGE LIWEASICAE WAT IONAL LAEDARTOFY
NEVADA- A Simulation Environment for Regional Estimation of Ground Motion and Structural Response

D. McCallen, S. Larsen

March 7, 2003 
This document was prepared as an account of work sponsored by an agency of the United States Government. Neither the United States Government nor the University of California nor any of their employees, makes any warranty, express or implied, or assumes any legal liability or responsibility for the accuracy, completeness, or usefulness of any information, apparatus, product, or process disclosed, or represents that its use would not infringe privately owned rights. Reference herein to any specific commercial product, process, or service by trade name, trademark, manufacturer, or otherwise, does not necessarily constitute or imply its endorsement, recommendation, or favoring by the United States Government or the University of California. The views and opinions of authors expressed herein do not necessarily state or reflect those of the United States Government or the University of California, and shall not be used for advertising or product endorsement purposes.

This work was performed under the auspices of the U.S. Department of Energy by University of California, Lawrence Livermore National Laboratory under Contract W-7405-Eng-48. 


\section{|||| NEVADA - A simulation environ- ment for regional estimation of ground motion and structural response}

\section{McCallen}

Laser Science Engineering Division Engineering Directorate

Lawrence Livermore National Laboratory

\section{January 2003}

A final report for the Laboratory Directed Research and Development Project \#02-ERD-044

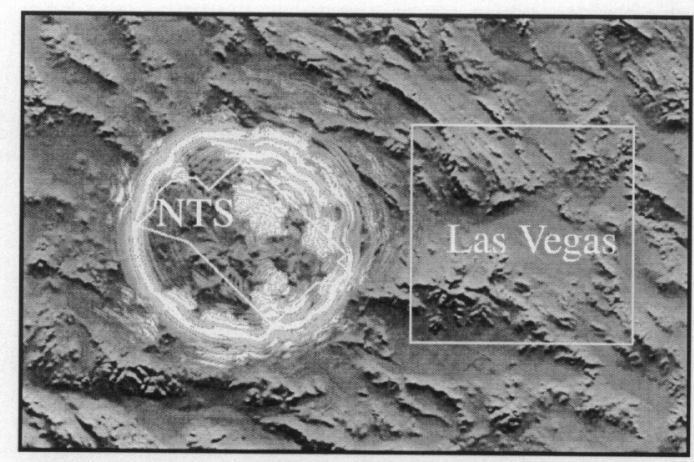

\section{S. Larsen}

Earth Sciences Group

Computations Directorate

Lawrence Livermore National Laboratory
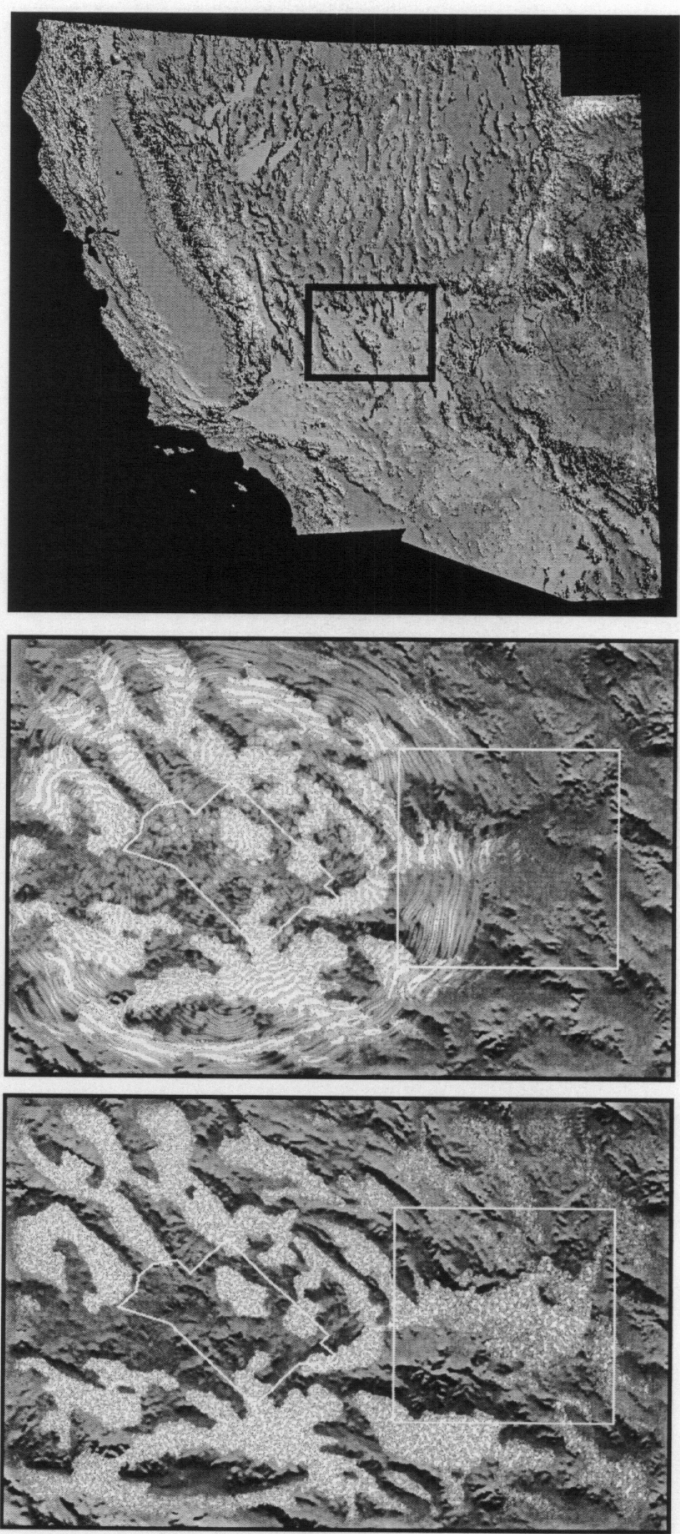

(1)

\section{U.S. Department of Energy}

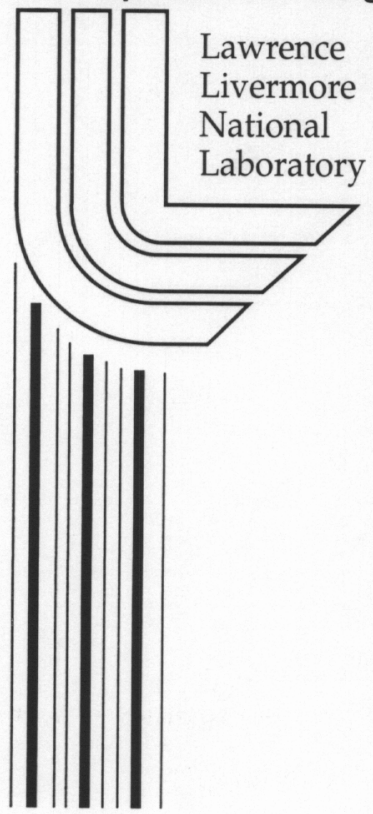

Lawrence

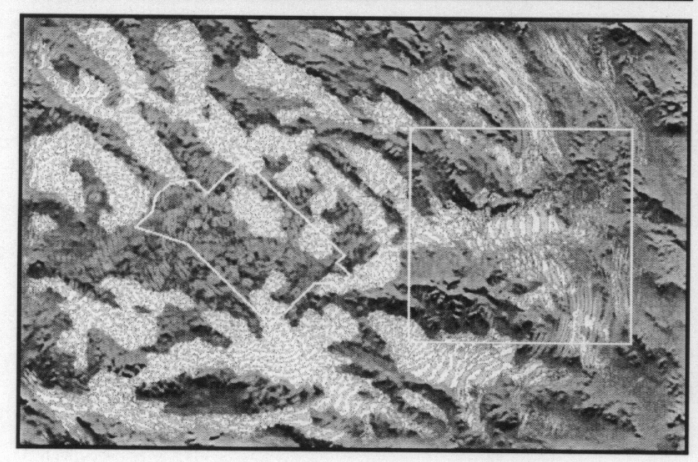




\section{NEVADA - A simulation environment for regional estimation of ground motion and structural response}

\section{Contents}

1.0 Background

2.0 Southern Nevada: a unique "laboratory" for regional response studies ...................3

3.0 The regional simulation environment ................................................................

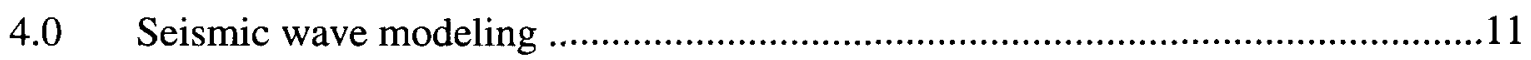

$4.1 \quad$ General equations.......................................................................................................11

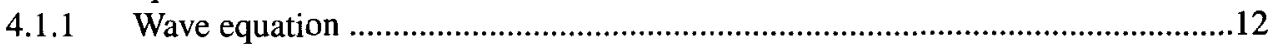

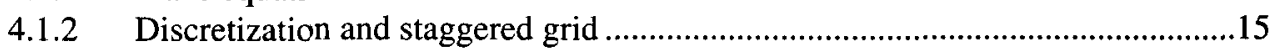

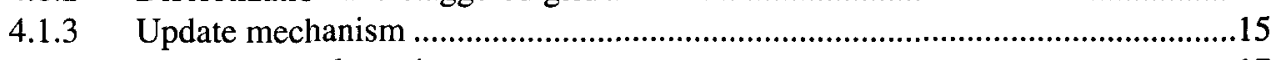

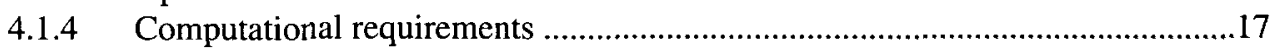

4.1.5 Geological representation (averaging)....................................................18

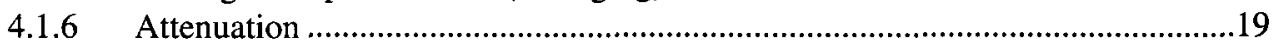

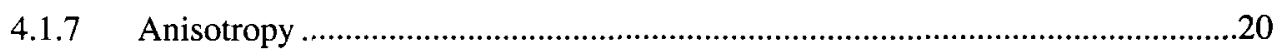

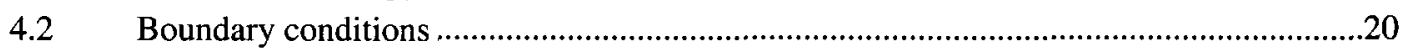

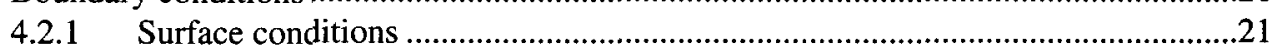

4.2.2 Paraxial side boundaries .........................................................................22

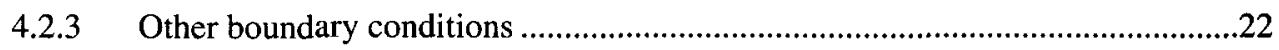

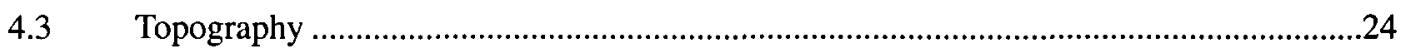

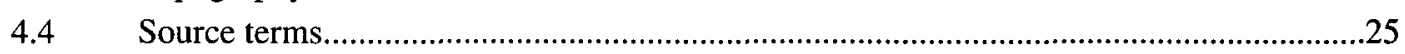

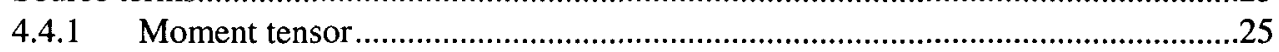

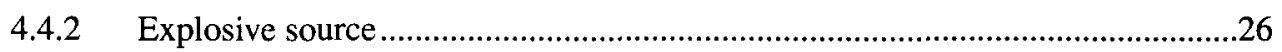

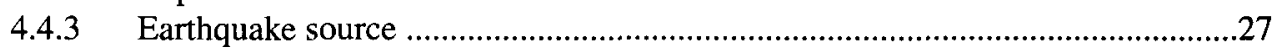

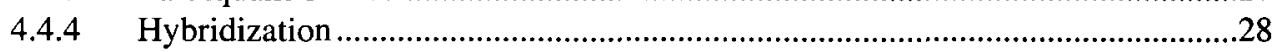

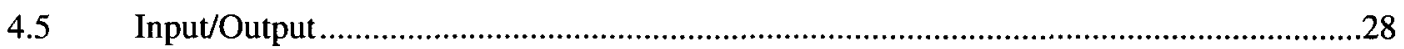

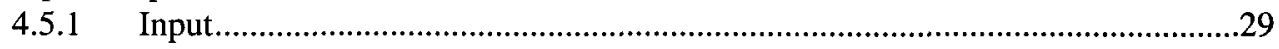

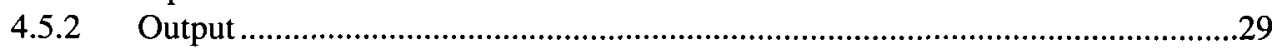

4.5.3 Receiver and output formulations.........................................................29

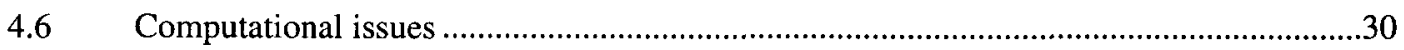

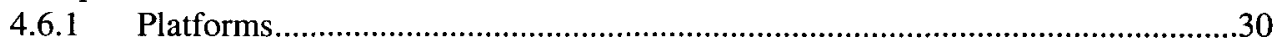

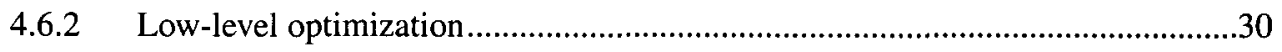

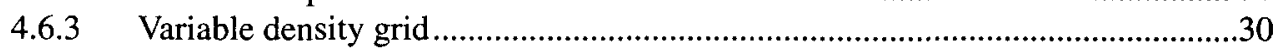

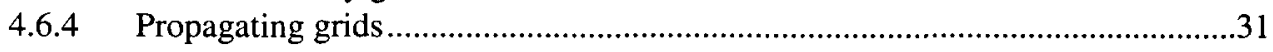

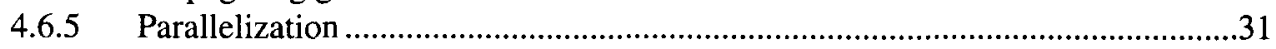

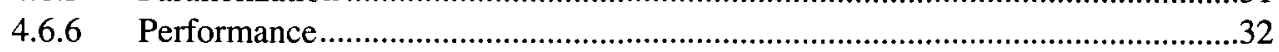

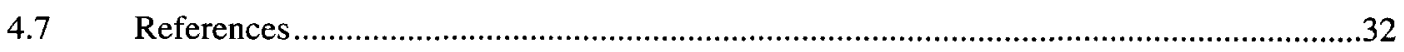

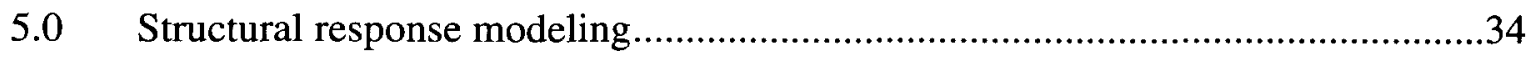

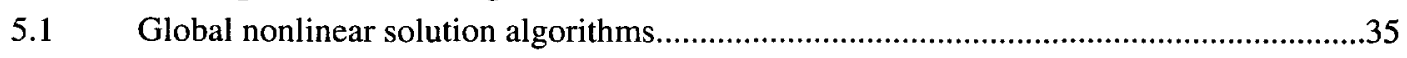

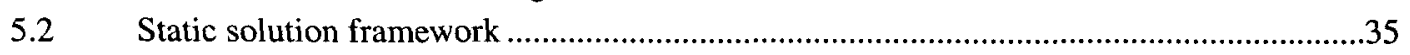




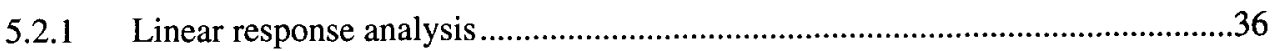

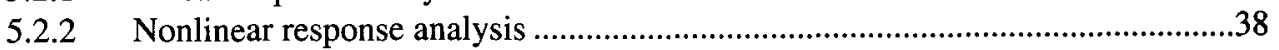

$5.2 .3 \quad$ Linear time-history analysis ........................................................................4

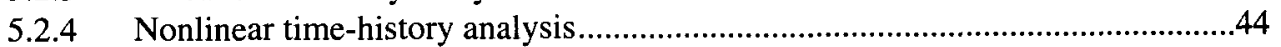

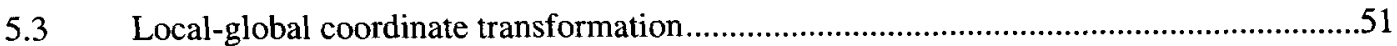

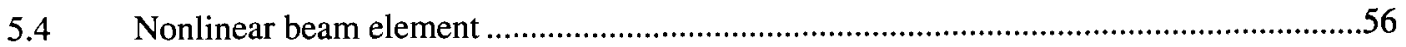

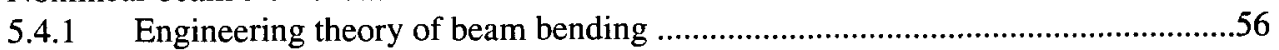

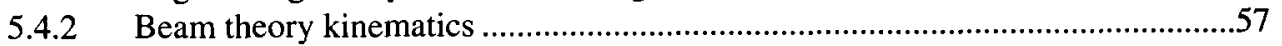

5.4.3 Beam theory stress resultants .........................................................................60

5.4.4 Beam finite element ...................................................................................63

5.4.5 Finite fiber beam cross section .....................................................................

5.4.6 Elasto-plastic constitutive model ....................................................................75

5.4.7 Implementation of the finite fiber elastoplastic element......................................76

$5.5 \quad$ Representation of steel structure plasticity ..................................................................

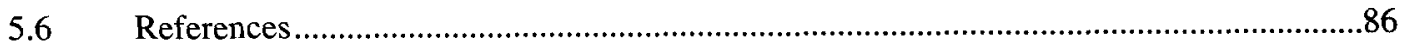

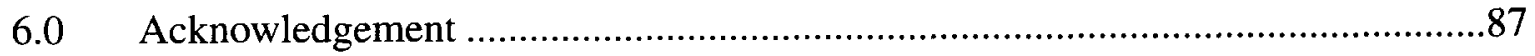

\section{APPENDIX 1}

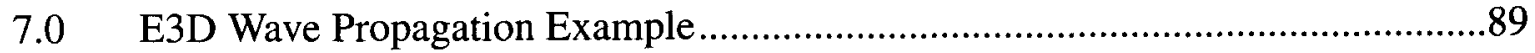

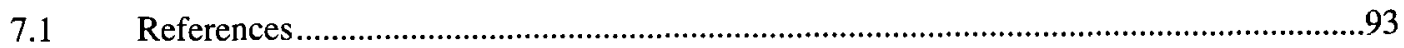

APPENDIX 2

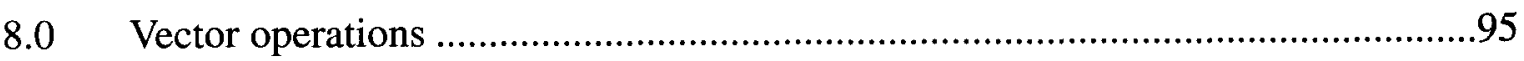

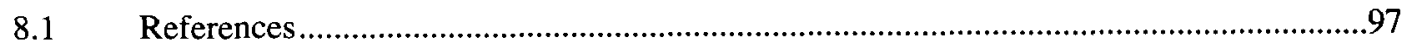

APPENDIX 3

9.0 Representative Steel Frame Structures .......................................................99

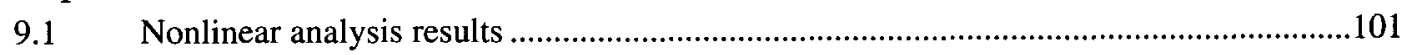

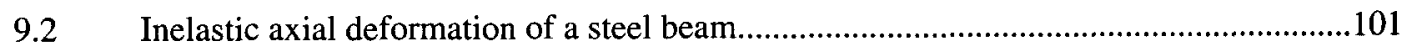

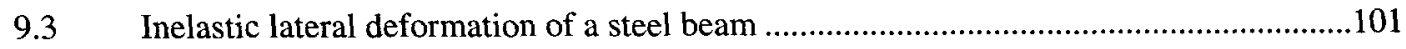

9.4 Linear and nonlinear analyses of a planar building ........................................................101 


\subsection{Background}

Energy released during natural earthquakes and underground man-made nuclear explosions manifests in the form of propagating seismic waves which radiate away from the energy source at high velocity. These propagating waves can generate significant motions at the ground surface for a widely distributed geographic area. Earthquakes and underground nuclear tests are both capable of generating ground motions levels that are potentially damaging to structures over a region of hundreds of square kilometers. To ensure adequate and economical protection of property and life-safety, it is essential that accurate predictions of ground motion levels and structural vibration amplitudes be generated for potential future events.

As seismic waves emanate from an energy releasing source, there are complex interactions between the waves and the highly heterogeneous geologic medium which constitutes the earth's upper crust. The waves reflect and refract at geologic discontinuities such that even for a very simple source function, e.g. a short duration explosive point source, the observed ground surface motions exhibit waveforms of significant complexity. The field deployment of modern seismic instrumentation has provided insight into the complex waveforms, the spatial variability of generated ground motions, and the dependence of observed motions on propagation path geology and local site soil conditions. For example, the observed amplification and extended time duration of ground motions in soft sedimentary basins, owing to the effects of low velocity near-surface soil sediments and trapping of the seismic wave energy in the basin, is of particular concern when estimating the seismic hazard for a particular region. A major scientific and engineering challenge is to accurately estimate the regional variation of ground motions, and to predict the expected response of structures to these motions throughout the region of interest.

In the work reported on herein, a numerical simulation based framework for regional estimation of ground motions and structural response is described. The computer simulation framework which has been developed provides a tool for regional scale simulation of seismic wave propagation and structural response. The simulation framework development is focused on a case study application of the Southern Nevada region encompassing the Nevada Test Site and Las Vegas. This region was choosen because a large database of regional ground motion measurements exist from the era of underground nuclear testing, and ground motion hazard in this region continues to be of interest to the National Nuclear Security Administration for future underground test readiness. This focus will provide a tool for immediate programmatic application of the research results in future DOE programs. However, once successfully developed, the methodologies described could readily be extended to application in any seismically active region.

\subsection{Southern Nevada: a unique "laboratory" for regional response studies}

From 1951 through 1992, 904 nuclear tests were conducted at the Nevada Test Site (NTS), located approximately 100-150 KM north of Las Vegas in southern Nevada (Figure 1). Of these tests, 100 were atmospheric events and 804 were underground events. Starting in 
1962, with the implementation of the atmospheric test ban, all nuclear tests at NTS were performed deep underground either in horizontal tunnels or vertical bore holes to achieve geologic containment of the event by-products. With the advent of underground testing, substantial amounts of energy were coupled into the geologic strata which effectively resulting in man-made earthquakes. Figure 2 provides graphic illustration of the enormous energy released by a 104KT device emplaced at shallow depth of burial in 1962 . The Sedan Plowshare event investigated the peaceful usage of nuclear devices for engineering construction purposes and the intent of the Sedan event was to demonstrate the ability to create a large man-made excavation.

During the underground testing era, particularly in the 1970's when large Megaton TNT equivalent events were being conducted at NTS ${ }^{1}$, the effects of event induced ground motions on major structures became of concern and a program was implemented to address the risk of structural damage. This concern was added to by the fact that Las Vegas is located in a deep sedimentary basin and ground motion measurements indicated that seismic waves propagating from NTS to the Las Vegas Valley were trapped and amplified in the low velocity sediments of the soft basin. To measure the event induced ground motions and assess structural response, a significant number of ground motion sensors and structural response sensors were deployed throughout the Las Vegas Valley by the Department of Energy. The extent of the historical ground motion and infrastructure instrumentation is shown in Figure 3. Throughout the years of underground testing, the motions from a large number of nuclear tests were captured on the Las Vegas instruments. The data from 13 representative events has been processed, digitized and written to compact disk by the Nevada Operations Office of the NNSA. This data, which encompasses a range of events in terms of explosive yield and source location, provides a unique suite of data on the response of a sedimentary basin and the response of structures in the basin on a regional scale. Given the large number of nuclear events, this regional response dataset is extensive when compared to natural earthquake data where only one or two major earthquakes have been measured owing to the infrequent nature of natural earthquakes.

The historical nuclear test data clearly illustrates the effects of sedimentary basin response in Las Vegas. Figure 3 shows ground acceleration time histories at four selected sites for the Barnwell underground nuclear test of 1989. The rock sites on the edges of the basin (site \#1 and site \#3) exhibit significantly smaller motions than sites located in the middle of the sedimentary basin (sites \#2 and \#4) despite the fact that these sites are nearly equidistant from the source location at NTS. This is clear evidence of the amplification of motion by the soft soils in the basin. One issue of concern for future NNSA test readiness interests is the fact that none of the historical ground motion sites were placed in what is now know to be the deepest part of the basin. The dark purple region in Figure 3 indicates the deepest portion of the basin as determined by recent gravimetry studies performed by the United States Geological Survey. The figure key indicates soil depth in kilometers. It is expected, and the existing dataset indicates it is indeed the trend, that the largest amplitude motions correspond to the deeper part of the basin. The northeastern portion of the basin

1. The largest megaton level underground tests (for example the NTS "Boxcar" event of 1.3 MT in 1968) generated energy release equivalent to a $M=6+$ natural earthquake. 


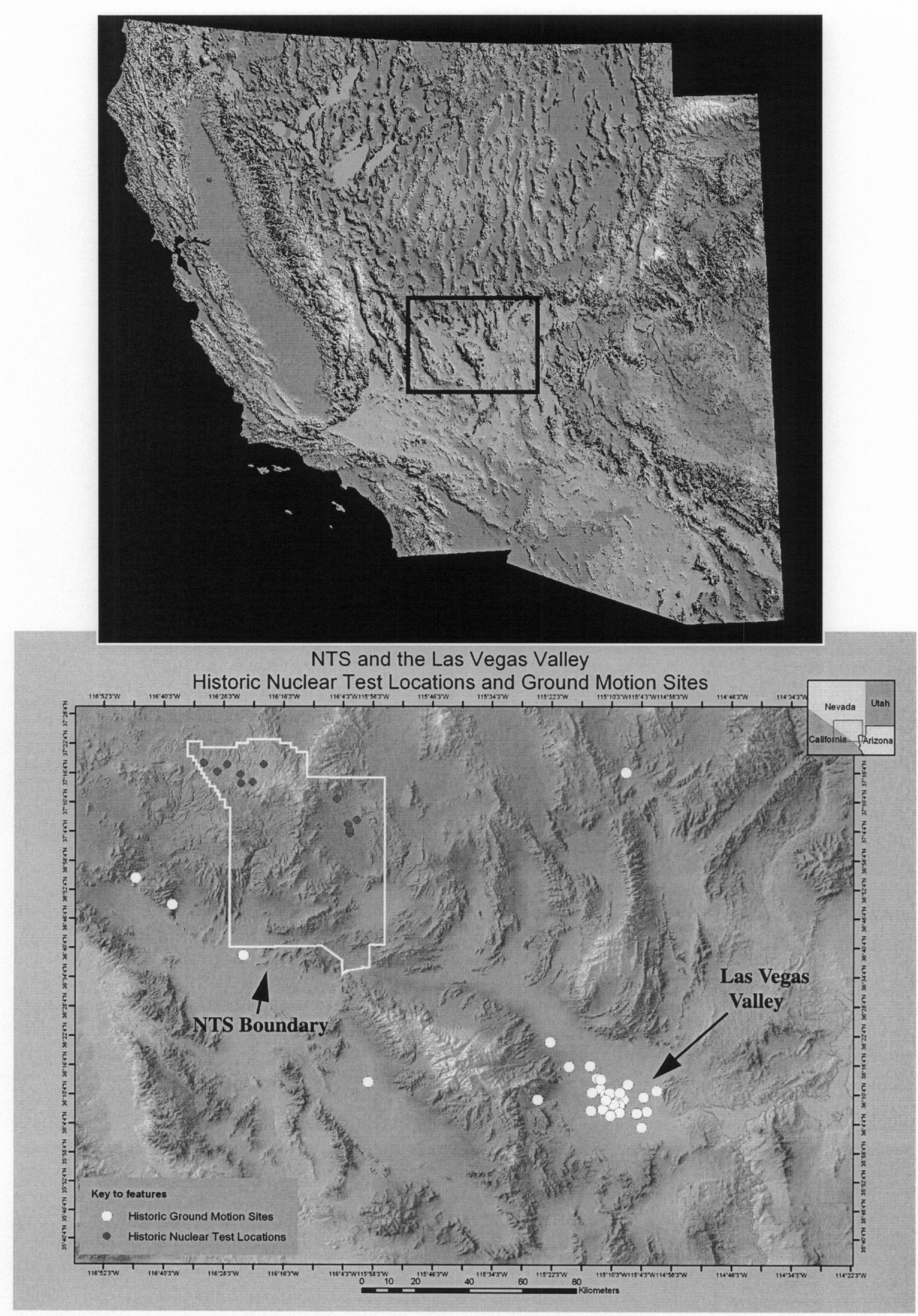

FIGURE 1. Regional geologic setting and location of NTS relative to the Las Vegas Valley. 
a)

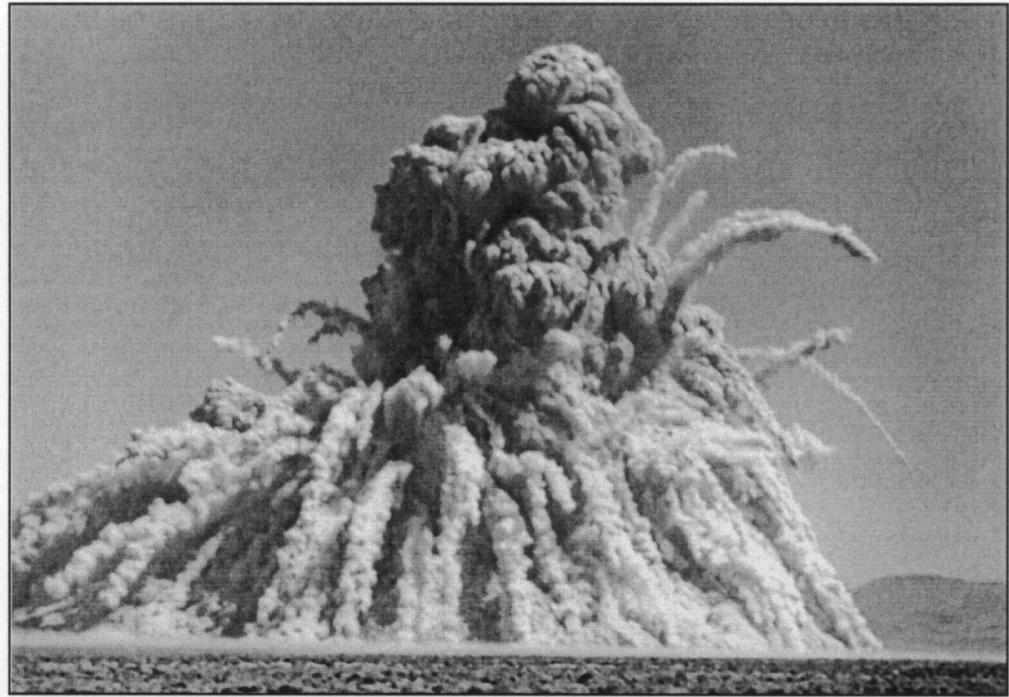

b)

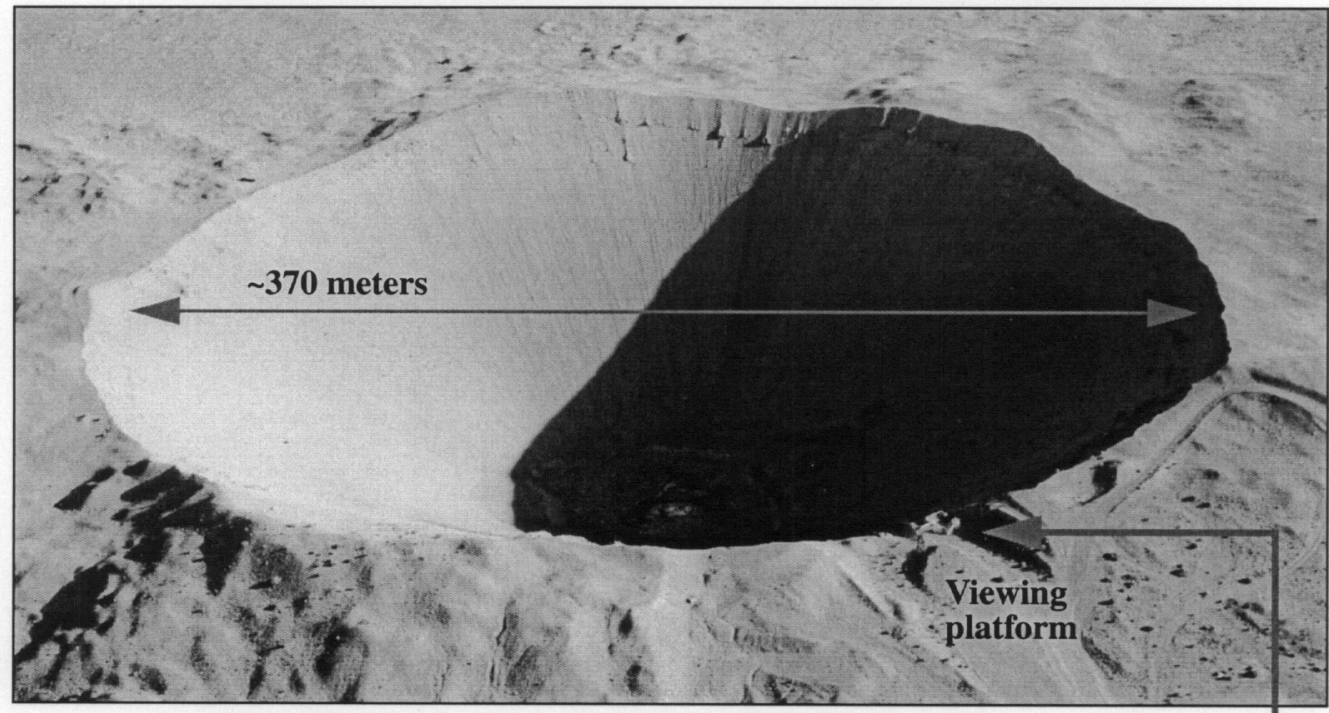

c)
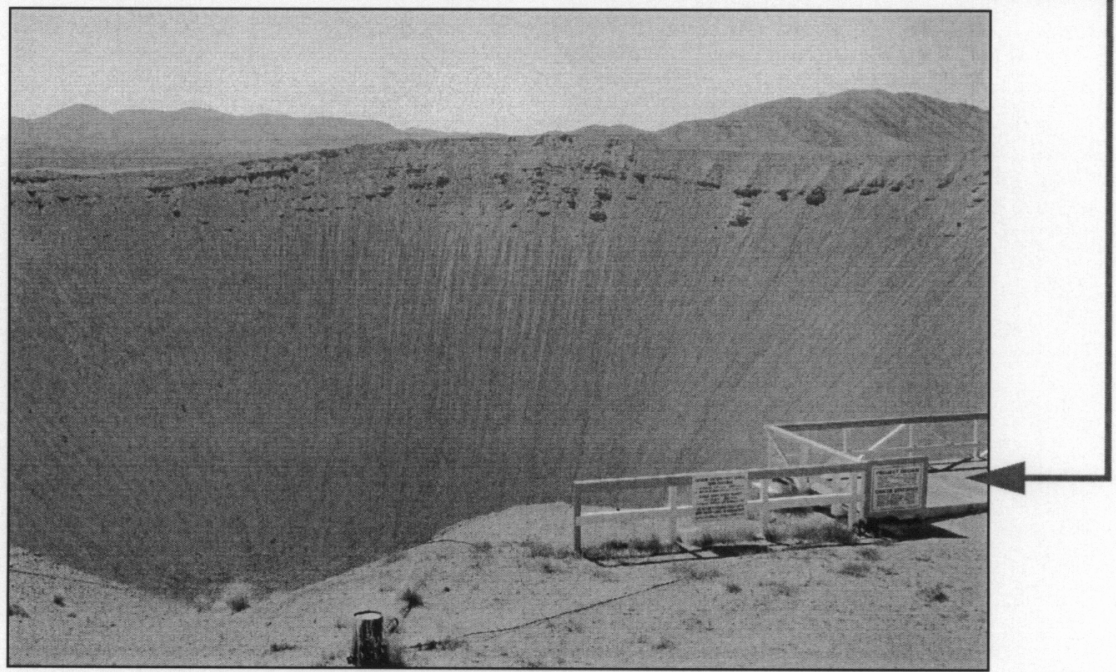

FIGURE 2. Creation of the Sedan crater from a 104 KT Plowshare device in 1962. a) Ejecta from detonation of device buried at $195 \mathrm{~m}$ depth; b) overhead view of resulting crater (370 meters in diameter, 97 meters deep); c) view from edge of crater. 


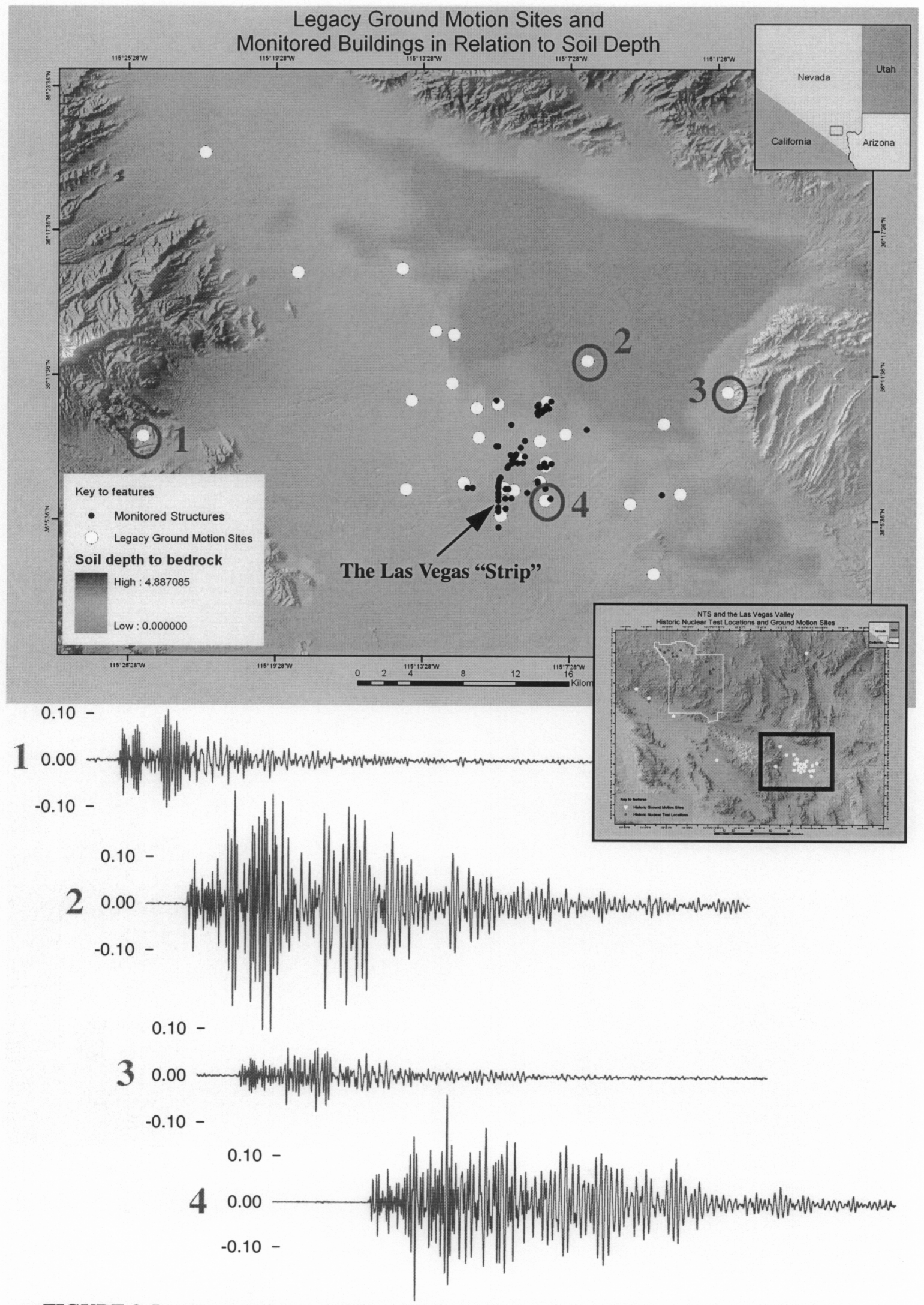

FIGURE 3. Instrumented ground sites and structures in the Las Vegas Valley (until 1992), ground accelerations from the Barnwell underground nuclear test of 1989. 
was largely undeveloped in the 1970's and unfortunately the legacy ground motion instrumentation network was not extended into this area. Estimation of the motions in this deepest part of the basin is an issue for close examination with the tools described in this report in future programmatic application of the tools.

The historical underground test data provides an important body of observational knowledge critical to validation and calibration of regional based models. Comparison of regional scale models with such regional data is essential to quantifying the ability of complex, large-scale regional models to provide accurate estimates of regional response. The historical underground test data incorporates many events with simple source functions at approximately the same distance and direction from Las Vegas. This eliminates the source complexities associated with extended-source natural earthquakes and allows investigation of trends within the large number of events available.

\subsection{The regional simulation environment}

The simulation environment incorporates geophysics and structural models into a massively parallel computational framework that permits regional calculation of ground motion and structural response. The regional simulation framework is named "NEVADA" in recognition of the first regional case study application. Ground motions are computed with the LLNL E3D elastic, three-dimensional finite difference wave propagation code, and structural response to ground motions is computed from a suite of Fortran subroutines tailored to the linear and nonlinear analysis of planar building structures. For regional scale three-dimensional problems, the geophysics computational domain is quite large with on the order of 400-500 million zones. This total domain is divided into subgrids with each subgrid devoted to a specific node on LLNL parallel compute engines. Once the ground motion simulation is completed for a specific event, the computed ground motions at selected Las Vegas Valley grid points are applied to the building structure under consideration. The building analyses are also performed in parallel with one building analyses delegated to each computer processor.

The ground motion hazard is characterized in a structure-specific fashion whereby a building designed pursuant to the appropriate building code is analyzed at predetermined grid points throughout the region. This directly defines the hazard in terms of building demands. Building demands are specified in terms of interstory drift ratios, i.e. the relative displacement between adjacent floor levels for each story of the building. Interstory drift demands from the building analyses can be readily compared to damage thresholds developed from destructive laboratory tests of building segments. This process is shown schematically in Figure 4 and Figure 5.

This report documents the theoretical foundation of the components of the regional simulation model. Future work will include detailed comparisons between the developed regional model and the historical test data. Sensitivity and parametric studies will also be performed with the developed regional model to ascertain how various parameters influence basin response. 


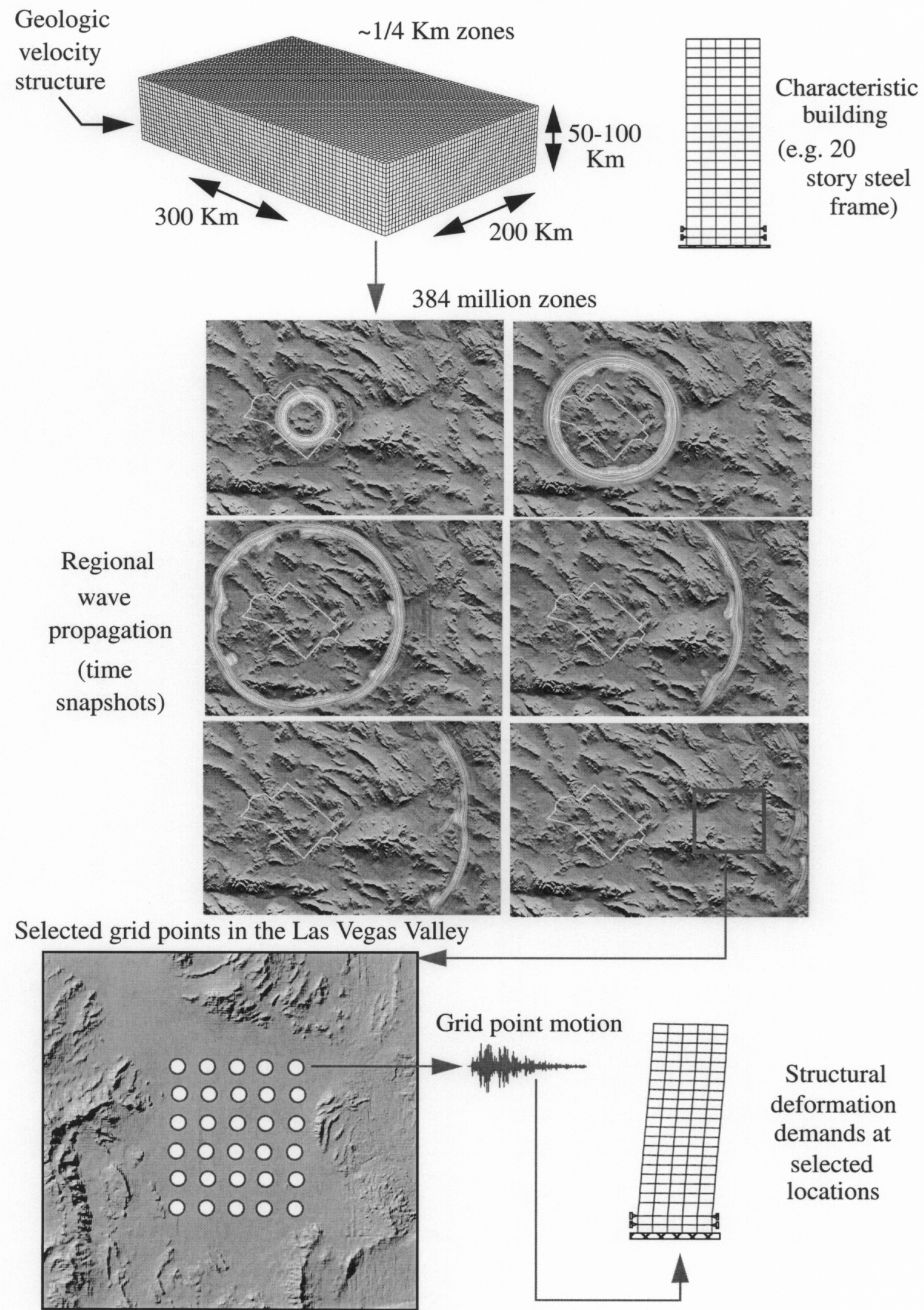

FIGURE 4. NEVADA solution framework for estimation of ground motions and structural response. 
a)
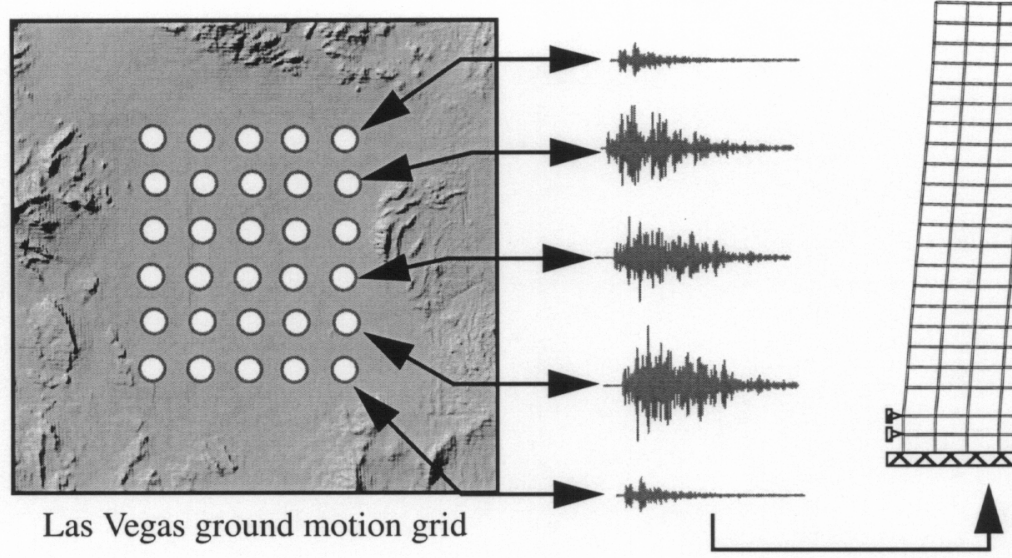

Structure

dynamic

response to ground

motions

Las Vegas ground motion grid

Individual grid

motions applied to building

b)

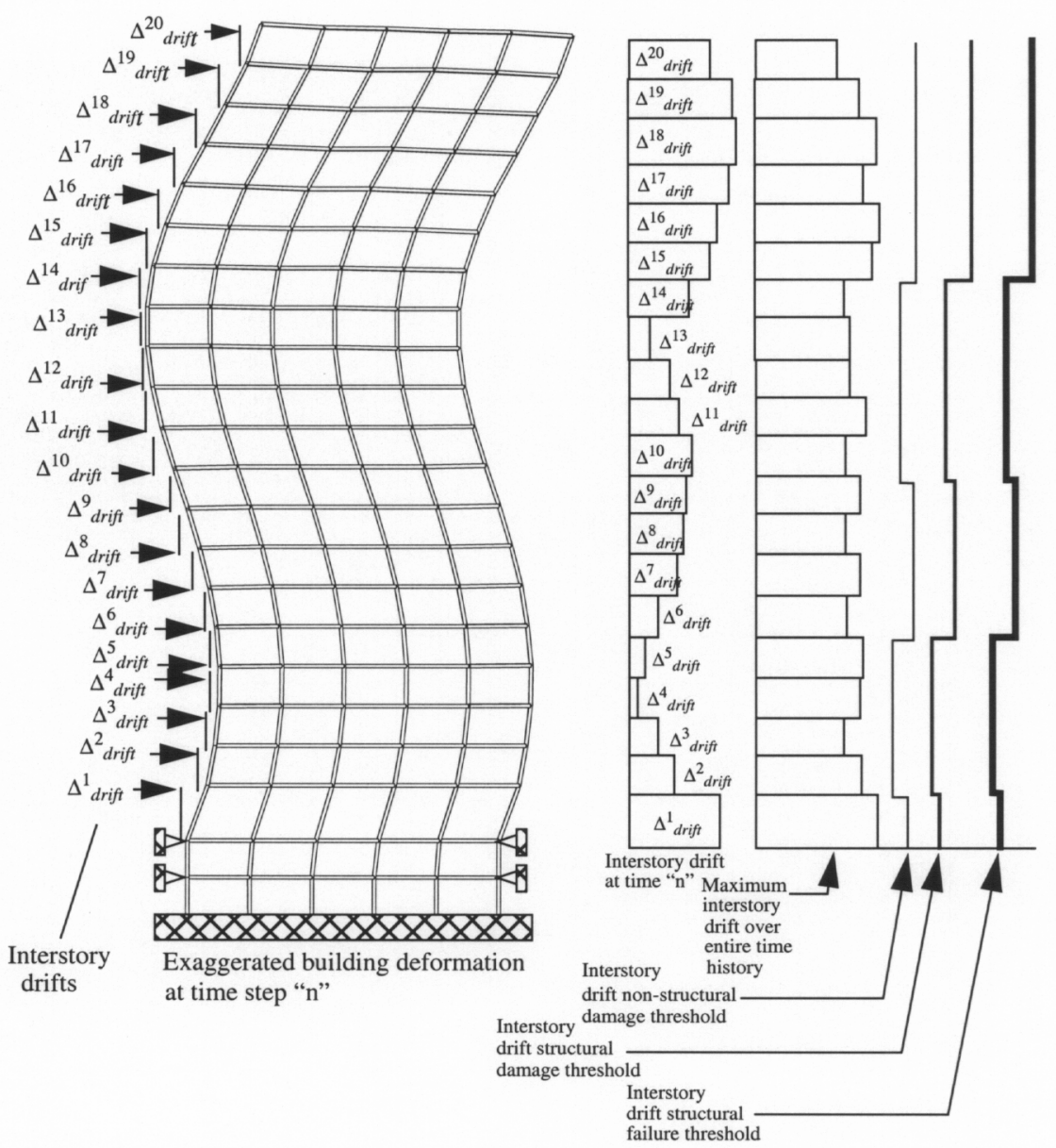

FIGURE 5. Structural demands in terms of interstory drifts. a) Computing drift demands throughout the region of interest; b) comparing drift demand envelope with damage thresholds established from structural testing. 


\subsection{Seismic wave modeling}

E3D is a 3-D finite-difference seismic wave propagation code that serves as a component of the NEVADA simulation environment. E3D simulates the propagation of elastic and acoustic energy generated by a source (e.g., nuclear explosion, earthquake) through a 3-D geologic model of the earth or other material (Figure 6). The surface ground motions computed by this propagation can be used to drive the response of buildings and other structures.

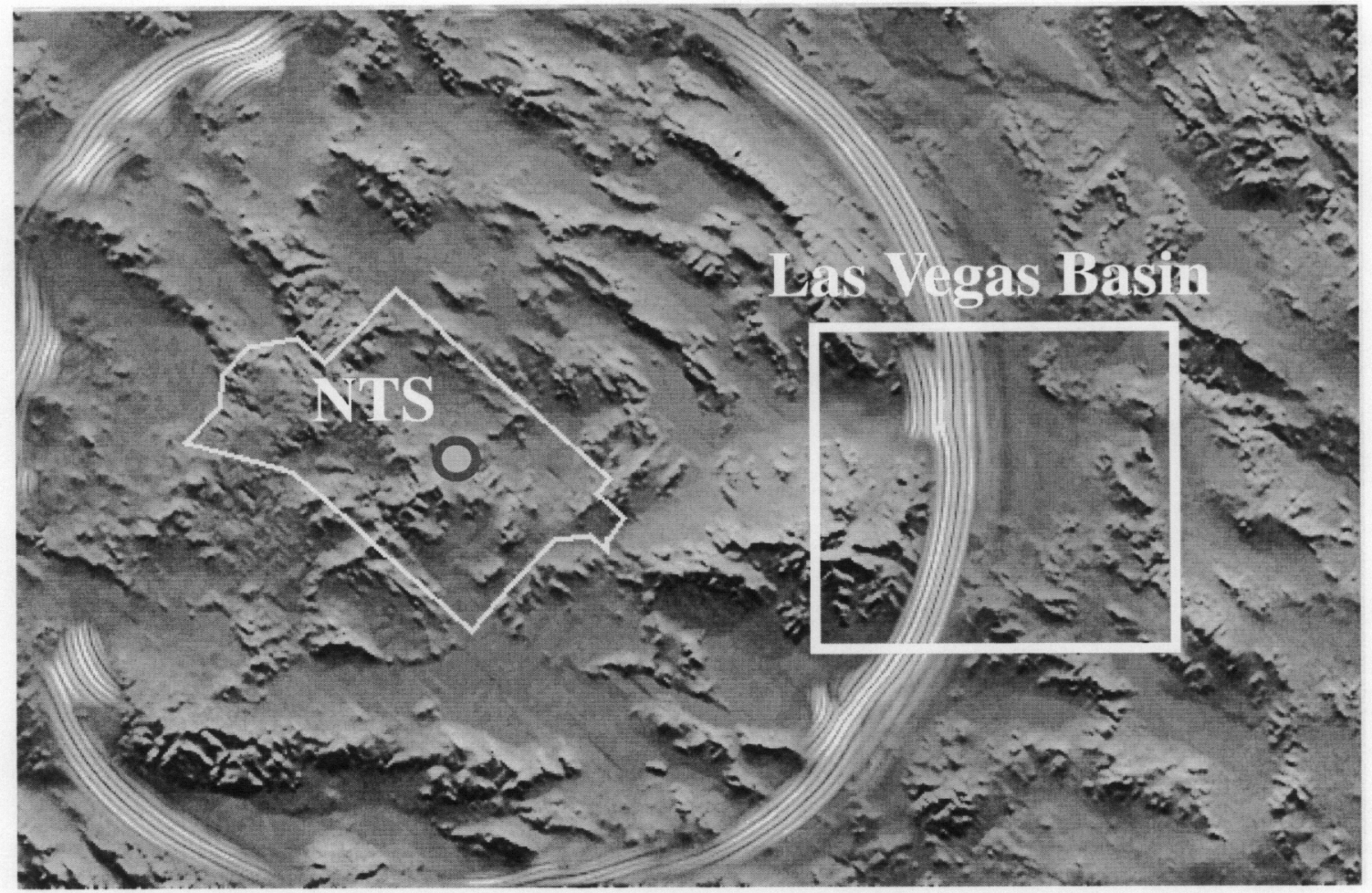

FIGURE 6. Seismic waves propagate from the Nevada Test Site (NTS) to the Las Vegas Basin (LVB).

E3D incorporates a number of advanced computational and physics-based features. These features include full 3-D elastic, viscoelastic, and topographic modeling, low-level optimization, propagating and variable density grids, hybridization, parallelization, and run-time visualization. This code runs on a number of computer platforms and takes advantage of high performance computing and massively parallel processing. This makes 3-D fullphysics simulations of large seismic problems feasible. In addition, E3D offers 2-D modeling capabilities, which often serve as a mechanism for prototyping larger three-dimensional simulations.

\subsection{General equations}

E3D is based on the elastodynamic formulation of the full wave equation on a staggered grid (e.g., Madariaga, 1976; Virieux, 1986; Levander, 1988). In this formulation, the 
velocities and stress tensor components are solved by an explicit finite-difference scheme. The focus here is on wave propagation in a three-dimensional earth; two-dimensional formulations are easily obtained by decomposing the three-dimensional equations.

\subsubsection{Wave equation}

A number of formulations are used to describe the linear propagation of wave energy in an acoustic or elastic medium such as air, water, earth, human tissue, or an engineering solid. For example, the 1-D propagation of acoustic energy in a water volume of constant medium velocity is given by the 2 nd-order partial differential equation

$$
\frac{\partial^{2} P}{\partial t^{2}}=C \cdot \frac{\partial^{2} P}{\partial x^{2}}
$$

where $P$ is the pressure at spatial coordinate $x$ and time $t$, and $C$ is the wave propagation velocity. In this case, the propagation velocity is the parameter that uniquely defines the medium. It can be a constant for simple problems, or it can be a function of the spatial position $(C(x))$. Similarly, the propagation of acoustic energy in 3-D is given by

$$
\frac{\partial^{2} P}{\partial t^{2}}=C(x, y, z) \cdot\left(\frac{\partial^{2} P}{\partial x^{2}}+\frac{\partial^{2} P}{\partial y^{2}}+\frac{\partial^{2} P}{\partial z^{2}}\right)
$$

where $P=P(x, y, z, t), C=C(x, y, z)$, and $x, y$, and $z$ are the spatial coordinates. More complex equations exist that allow $C$ to be a function of the density of the medium.

Wave propagation in the solid earth depends on acoustic and elastic properties. The 3-D elastodynamic formulation of the wave equation is given by the set of 2 nd order equations

$$
\begin{aligned}
& \frac{\partial^{2} U_{x}}{\partial t^{2}}=1 / \rho \cdot\left(\frac{\partial \tau_{x x}}{\partial x}+\frac{\partial \tau_{x y}}{\partial y}+\frac{\partial \tau_{x z}}{\partial z}+F_{x}\right)
\end{aligned}
$$

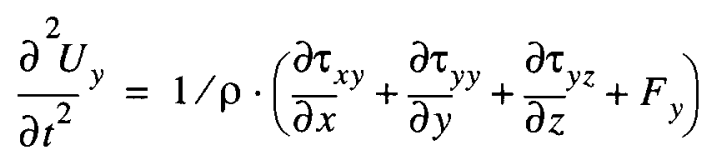

$$
\begin{aligned}
& \frac{\partial^{2} U_{z}}{\partial t^{2}}=1 / \rho \cdot\left(\frac{\partial \tau_{x z}}{\partial x}+\frac{\partial \tau_{y z}}{\partial y}+\frac{\partial \tau_{z z}}{\partial z}+F_{z}\right)
\end{aligned}
$$

and 


$$
\begin{aligned}
& \tau_{x x}=(\lambda+2 \mu) \cdot \frac{\partial U_{x}}{\partial x}+\lambda \cdot\left(\frac{\partial U}{\partial y}^{y}+\frac{\partial U_{z}}{\partial z}\right)+S_{x x} \\
& \tau_{y y}=(\lambda+2 \mu) \cdot \frac{\partial U}{\partial y}^{y}+\lambda \cdot\left(\frac{\partial U}{\partial x}_{x}+\frac{\partial U_{z}}{\partial z}\right)+S_{y y} \\
& \tau_{z z}=(\lambda+2 \mu) \cdot \frac{\partial U_{z}}{\partial z}+\lambda \cdot\left(\frac{\partial U_{x}}{\partial x}+\frac{\partial U_{y}}{\partial y}\right)+S_{z z} \\
& \tau_{x y}=\mu \cdot\left(\frac{\partial U}{\partial y}^{x}+\frac{\partial U^{y}}{\partial x}\right)+S_{x y} \\
& \tau_{x z}=\mu \cdot\left(\frac{\partial U_{x}}{\partial z}+\frac{\partial U_{z}}{\partial x}\right)+S_{x z} \\
& \tau_{y z}=\mu \cdot\left(\frac{\partial U_{y}}{\partial z}+\frac{\partial U_{z}}{\partial y}\right)+S_{y z}
\end{aligned}
$$

where $U x, U y$, and $U z$ are particle displacements of the ground and $\tau_{x x}, \tau_{y y}, \tau_{z z}, \tau_{x y}, \tau_{x z}$ and $\tau_{y z}$ are the 6 components of the symmetric stress tensor. The parameters $\rho, \lambda$ and $\mu$ uniquely describe the medium, which in this case is the geology of the subsurface. In addition, $U=U(x, y, z, t), \tau=\tau(x, y, z, t), \rho=\rho(x, y, z)$, $\lambda=\lambda(x, y, z), \mu=\mu(x, y, z)$. The terms $F x, F y, F z$, and $S x x, S y y, S z z, S x y, S x z$, and $S y z$ are forcing or driving functions that will be discussed later.

The acoustic or compressional velocity $V p$ in the medium is given by

$$
V p=\sqrt{(\lambda+2 \cdot \mu) / \rho}
$$

and that the shear velocity $V s$ is given by

$$
V p=\sqrt{\mu / \rho}
$$

These relationships are most useful because seismologists and geophysicists often define medium properties in terms of the rock density and the propagation velocity of seismic waves.

In essence, EQ. 3a through EQ. 3c represent the momentum equations $(F=M A)$, while EQ. 3d through EQ. 3f represent the constitutive relations or Hooke's Law $(F=k X)$. This coupled set of 2 nd-order partial differential equations can be written as a coupled set of 
1st-ordered differential equations by using the relationship $V=d U / d t$ and taking derivatives with respect to time. Hence,

$$
\begin{aligned}
& {\frac{\partial V_{x}}{\partial t}}^{x}=1 / \rho \cdot\left(\frac{\partial \tau_{x x}}{\partial x}+\frac{\partial \tau_{x y}}{\partial y}+\frac{\partial \tau_{x z}}{\partial z}+F_{x}\right) \\
& \frac{\partial V_{y}}{\partial t}=1 / \rho \cdot\left(\frac{\partial \tau_{x y}}{\partial x}+\frac{\partial \tau_{y y}}{\partial y}+\frac{\partial \tau_{y z}}{\partial z}+F_{y}\right) \\
& \frac{\partial V_{z}}{\partial t}=1 / \rho \cdot\left(\frac{\partial \tau_{x z}}{\partial x}+\frac{\partial \tau_{y z}}{\partial y}+\frac{\partial \tau_{z z}}{\partial z}+F_{z}\right)
\end{aligned}
$$

and

$$
\begin{aligned}
& \frac{\partial \tau}{\partial t}_{x x}=(\lambda+2 \mu) \cdot \frac{\partial V_{x}}{\partial x}+\lambda \cdot\left(\frac{\partial V_{y}}{\partial y}+\frac{\partial V_{z}}{\partial z}\right)+M_{x x} \\
& \frac{\partial \tau}{\partial t}_{y y}=(\lambda+2 \mu) \cdot \frac{\partial V}{\partial y}_{y}+\lambda \cdot\left(\frac{\partial V}{\partial x}^{x}+\frac{\partial V}{\partial z}\right)+M_{y y} \\
& \frac{\partial \tau_{z z}}{\partial t}=(\lambda+2 \mu) \cdot \frac{\partial V_{z}}{\partial z}+\lambda \cdot\left(\frac{\partial V_{x}}{\partial x}+\frac{\partial V_{y}}{\partial y}\right)+M_{z z} \\
& \overline{\partial \tau}_{x y}=\mu \cdot\left(\frac{\partial V}{\partial y}_{x}+\frac{\partial V_{y}}{\partial x}\right)+M_{x y} \\
& \frac{\partial \tau}{\partial t}_{x z}=\mu \cdot\left(\frac{\partial V}{\partial z}_{x}+\frac{\partial V_{z}}{\partial x}\right)+M_{x z} \\
& \overline{\partial \tau}_{\partial z}=\mu \cdot\left(\frac{\partial V}{\partial z}_{y}+\frac{\partial V_{z}}{\partial y}\right)+M_{y z}
\end{aligned}
$$

where $V$ is the particle or ground velocity as a function of space and time $(V=V(x, y, z, t))$, and the source term $M=d S / d t$. Note that $V x, V y$, and $V z$ are state variables (along with $T x x, T y y, T z z, T x y, T x z$, and $T y z$ ), which are different than the wave propagation velocities $V p$ and $V s$ that partially define the medium.

Equation 5a through EQ. 5i defines the set of 1st-order partial differential equations used by E3D to simulate wave propagation in three-dimensions. This particular representation 
is extremely useful because the equations are staggered in time, and the state variables can be staggered in space. Note that the velocities in EQ. 5a through EQ. 5c depend only on the stresses, and that the stresses in EQ. 5d through EQ. 5i depend only on the velocities. Hence, a valid numerical scheme is to explicitly update the velocities based on the stresses at the previous time, and then use the new velocities to update the stresses at the next time.

\subsubsection{Discretization and staggered grid}

Equation 5a through EQ. 5i are numerically solved by discretely approximating the equations on a regular but staggered grid. An explicit updating scheme is used to advance the solution in time. The spacing between identical variables in the grid is given by $d h$, which is the same in all three dimensions.

A staggered grid is used so that the known variables in EQ. 5a through EQ. 5i are centered around the variable being solved. For example, in EQ. 5a, the discrete grid can be spatially organized so that the variables $T x x, T x y$, and $T x z$ are centered around the variable $V x$. The spatial staggering is $1 / 2$ of the grid spacing in one or more dimensions (Figure 7). In addition, the equations allow the variables to be staggered in time. The known variables in Figure 5a ( $T x x, T x y$, and $T x z$, which are computing during a previous step), are staggered by $1 / 2$ timestep from the variable being solved during the update $(V x)$ and the same variable computed at the previous time. The spatial and temporal staggering permits higher numerical accuracy using a coarser grid (i.e., a grid with smaller computational requirements).

In E3D, the grid is constructed so that the normal stresses (Txx, Tyy, and Tzz) fall on the same staggered grid point, and this point is central to the grid system. The other variables are shifted by $1 / 2$ grid point in one or more directions. The medium parameters $\lambda, \mu$, and $\rho$ are coincident with this central point, although there are situations when this is not desired (discussed in a later section).

Unless otherwise stated, integer indices are used when referring to all grid points. For example, grid point $V x=V x(i, j, k, t)$ refers to the velocity variable $1 / 2$ grid point in the $x$ direction from the variables $T x x$, Tyy, and $T z z$ at grid indices $i, j, k$, and time $t$, even though a more robust terminology would refer to this variable as $V x(i+1 / 2, j, k, t)$. This formulation is used because this is how it is represented in computer memory (array indices must be integers).

\subsubsection{Update mechanism}

An explicit finite difference scheme is used to update EQ. 5a through EQ. 5i. In particular, E3D uses a scheme that is 4th-order accurate in space and 2nd-order accurate in time. The update equations are given by

$$
\begin{aligned}
& V_{x}(i, j, k, t)=V_{x}(i, j, k, t-1)+d t \cdot\left(D 4\left(T_{x x}\right)+D 4\left(T_{x y}\right)+D 4\left(T_{x z}\right)+F_{x}\right) / R \text { (EQ 6a) } \\
& V_{y}(i, j, k, t)=V_{y}(i, j, k, t-1)+d t \cdot\left(D 4\left(T_{x y}\right)+D 4\left(T_{y y}\right)+D 4\left(T_{y z}\right)+F_{y}\right) / R \text { (EQ 6b) }
\end{aligned}
$$




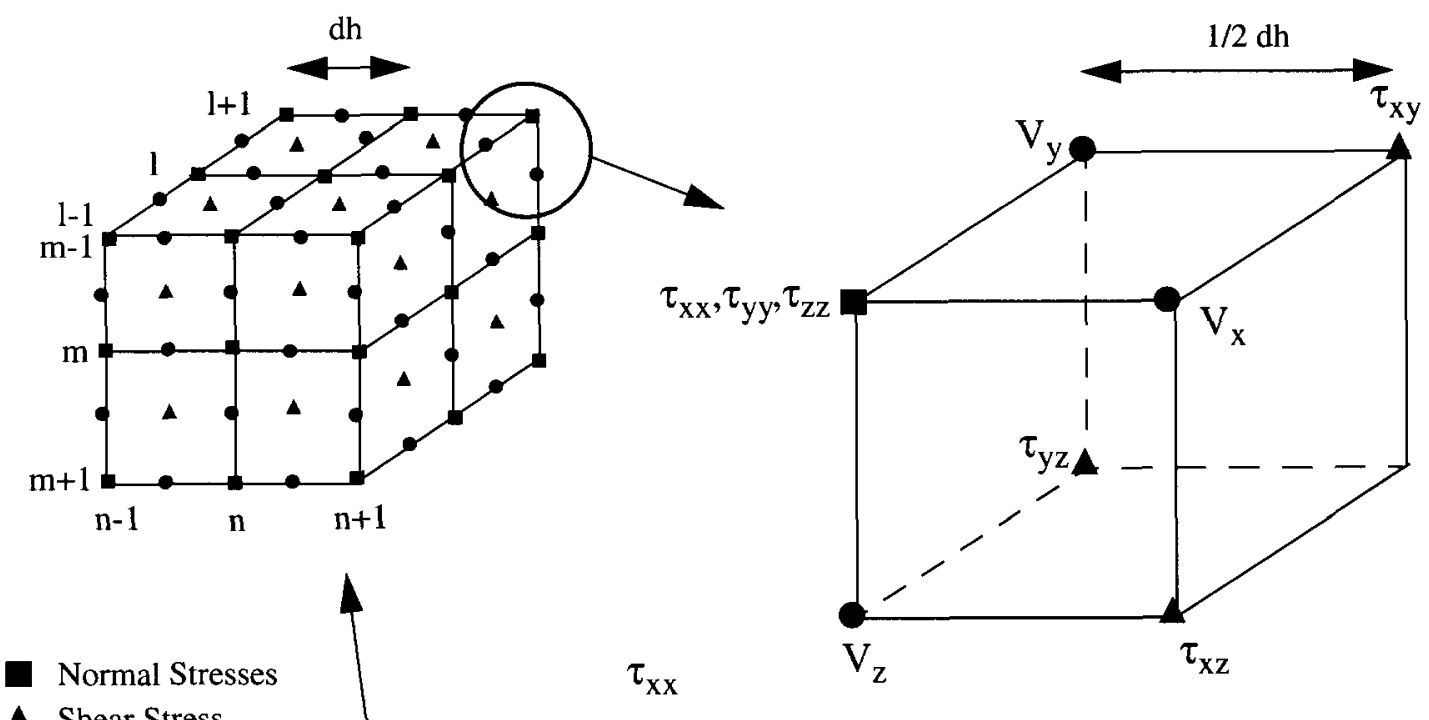

A Shear Stress

Velocities
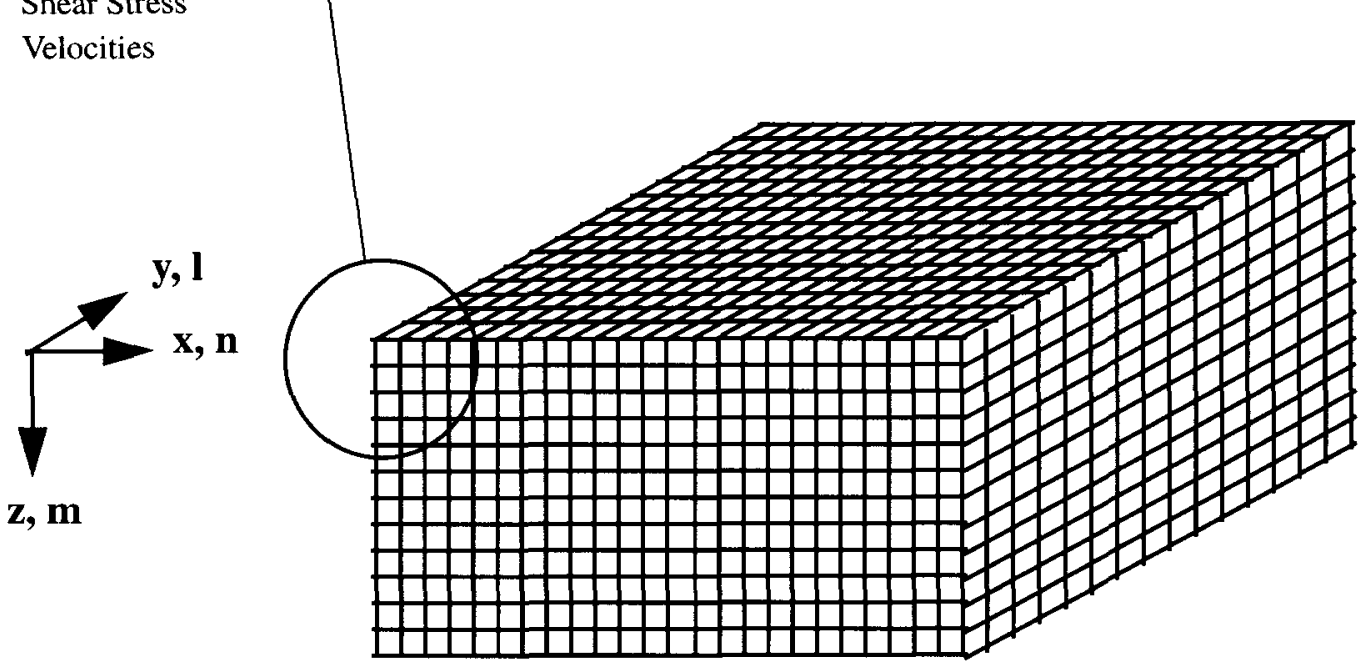

FIGURE 7. Geometry of staggered grid system.

$V_{z}(i, j, k, t)=V_{z}(i, j, k, t-1)+d t \cdot\left(D 4\left(T_{x z}\right)+D 4\left(T_{y z}\right)+D 4\left(T_{z z}\right)+F_{z}\right) / R$ (EQ 6c) and

$$
\begin{aligned}
& T_{x x}(i, j, k, t)=T_{x x}(i, j, k, t-1)+ \\
& d t \cdot\left(E 1 \cdot D 4\left(V_{x}\right)+E 2 \cdot\left(D 4\left(V_{y}\right)+D 4\left(V_{z}\right)\right)+M_{x x}\right) \\
& T_{y y}(i, j, k, t)=T_{y y}(i, j, k, t-1)+ \\
& d t \cdot\left(E 1 \cdot D 4\left(V_{y}\right)+E 2 \cdot\left(D 4\left(V_{x}\right)+D 4\left(V_{z}\right)\right)+M_{y y}\right)
\end{aligned}
$$




$$
\begin{gathered}
T_{z z}(i, j, k, t)=T_{z z}(i, j, k, t-1)+ \\
d t \cdot\left(E 1 \cdot D 4\left(V_{z}\right)+E 2 \cdot\left(D 4\left(V_{x}\right)+D 4\left(V_{y}\right)\right)+M_{z z}\right) \\
T_{x y}(i, j, k, t)=T_{x y}(i, j, k, t-1)+d t \cdot\left(E 3 \cdot\left(D 4\left(V_{x}\right)+D 4\left(V_{y}\right)\right)+M_{x y}\right) \\
T_{x z}(i, j, k, t)=T_{x z}(i, j, k, t-1)+d t \cdot\left(E 3 \cdot\left(D 4\left(V_{x}\right)+D 4\left(V_{z}\right)\right)+M_{x z}\right) \\
T_{y z}(i, j, k, t)=T_{y z}(i, j, k, t-1)+d t \cdot\left(E 3 \cdot\left(D 4\left(V_{y}\right)+D 4\left(V_{z}\right)\right)+M_{y z}\right)
\end{gathered}
$$

where $d t$ is the finite-difference sampling rate in time and

$$
\begin{aligned}
& E 1=\lambda(i, j, k)+2 . * \mu(i, j, k) \\
& E 2=\lambda(i, j, k) \\
& E 3=\mu(i, j, k) \\
& \mathrm{R}=\rho(\mathrm{i}, \mathrm{j}, \mathrm{k}) \\
& \text { and }
\end{aligned}
$$

$D 4(W)$ is the 4 th-order spatial difference of the function $W$ given by

$$
D 4(W)=C 1 \cdot(W(i, j, k, t)-W(i, j, k, t))+C 2 \cdot(W(i, j, k, t)-W(i, j, k, t))
$$

where $C 1$ and $C 2$ are 4th-order finite-difference coefficients given by

$$
C 1=1.125000000(9 . / 8)
$$

and

$C 2=-0.041666667(-1 . / 24)$

\subsubsection{Computational requirements}

Equation 6a through EQ. 6i operate over a discretized domain. The number of grid elements or nodes required for a simulation is a function of the problem size and the grid spacing $d h$. The problem size depends on the scientific region of interest. The grid spacing depends on the ability to numerically approximate the wave equation at sufficient accuracy. As a rule of thumb, it is desired that there be at least 5 and preferably 10 grid points per minimum source wavelength in the simulation.

The number of grid points or grid nodes required for a 3-D simulation is $L \cdot M \cdot N$, where $L, M$, and $N$ represent the number of grid points in the $x, z$, and $x$ directions, respectively. For purely elastic problems, 12 variables are required at each grid node for 3-D simulations ( 8 variables for $2-\mathrm{D}$ problems). In almost all situations, sufficient precision is satis- 
fied using 32-bit floating point arithmetic, or 4 8-bit words. Hence, 3-D simulations require $4 \cdot 12 \cdot L \cdot M \cdot N$ bytes of computer memory. This makes it possible to perform simulations on the order of $200 \times 200 \times 200$ grid points (384 MBytes) on a typical desktop workstation, $1000 \times 1000 \times 1000$ grid points (48 GBytes) on a moderate parallel machine, and $5000 \times 5000 \times 5000$ grid points ( 8 TBytes) on a world class computer system.

The time step interval $d t$ depends on the Courant condition, which is the maximum timestep that can stably propagate seismic waves in the medium. The Courant condition depends on the maximum geologic velocity in the medium $(V \max )$, as well as the grid spacing. The Courant condition is given by

$$
d t<(C F \cdot d h) /(\operatorname{Vmax})
$$

where $C F=0.606$ for 2-D problems and $C F=0.494$ for 3-D problems. In general, it is desired that the timestep increment be as close as possible to the Courant limit.

Depending on how the algorithm is structured, 2-D simulations may require 64 floating point operations per grid point at each time step, and 3-D simulations may require 141 floating point operations per grid point at each time step. Typical 3-D simulations require 1 to 20 hours of simulation time on a computer system appropriate for the problem size.

It is important to note that for 3-D simulations, memory scales as the $3 r d$ power of problem size, while simulation time scales as the 4 th power of problem size. Hence, doubling the dimensions of the problem, doubling the source frequency, or decreasing the minimum propagation velocity in the medium will require an 8-fold increase in computer memory and a 16-fold increase in simulation time. For 2-D problems, memory scales as the square of problem size, while simulation time scales as the cube of problem size.

\subsubsection{Geological representation (averaging)}

The medium parameters $(\rho, \lambda, \mu)$ are normally defined at the central location of the staggered grid. That is, they are defined at the same grid element that contains the normal stress variables $(T x x, T y y, T z z)$. This definition is used as a means to minimize computer memory and CPU time. However, this is not always desirable because other representations tend to produce more accurate results.

For more accurate simulations, the medium parameters can be defined at the grid element containing the variable being updated. For example, in EQ. 5a, the density ( $\rho$ ) can be defined on the grid element containing the $V x$ variable. Two methods are used to implement such a scheme. In the first, the density $\rho(i+1 / 2, j, k)$ can be obtained by simply averaging the densities between adjoining grid points. That is, $\rho(i+1 / 2, j, k)=(\rho(i, j, k)+\rho(i+1, j, k)) / 2$. This averaging is required at each time step in the simulation, and hence it can require a significant increase in CPU time. In the 2nd method, the densities are defined and stored at each of the appropriate grid points. That is, there are separate density values assigned at the $V x, V y$, and $V z$ grid elements. This permits faster execution, but requires significantly more computer memory. Similarly, the 
$\mu$ values can be defined at each of the three shear stress elements (Txy, Txz, and Tyz) by simply averaging these values at each timestep (harmonic averaging is used in practice), or by storing these values in separate memory locations for the entire simulation.

Medium averaging is normally not performed because of the increased CPU time and/or memory requirement. When it is performed, a cost analysis must be made to determine which averaging scheme is optimal.

\subsubsection{Attenuation}

Seismic waves are intrinsically attenuated as mechanical energy is converted into heat as the waves propagate through the earth. This attenuation can be small and insignificant, which is often the case for waves propagating through rock, or it can be large and important, which is often the case for waves propagating through soft sedimentary material characteristic of basins and the near surface.

Seismologists use an attenuation factor, or $Q$, for quantifying the magnitude of seismic attenuation. $Q$ is defined such that the amplitude of an attenuated seismic wave and is given by

$$
A=A_{0} \cdot e^{-\left(\frac{\pi \cdot x}{Q \cdot \lambda}\right)}
$$

where $A_{O}$ is the original amplitude, $\lambda$ is the wavelength, and $x$ is the distance in wavelengths the wave has traveled. In others words, $Q$ is the number of wavelengths a wave must travel before it's amplitude is reduced by a factor of $e^{-\pi}$. If $Q$ is large, the attenuation is small. If $Q$ is small, the attenuation is large. $Q$ is a property of the medium, similar to the density or the compressional or shear velocities. Often, there are separate $Q$ definitions for $\mathrm{P}$ and $\mathrm{S}$ wave energy (i.e., $Q p$ and $Q s$ ). $Q$ is most often considered to be independent of frequency. Since the attenuation depends on the attenuation and the frequency, this means that high frequency energy tends to be attenuated more than low frequency energy.

In E3D, attenuation is approximated using relaxation mechanisms and memory variables [e.g., Robertsson et al., 1994; Blanch et al., 1995]. Basically, one or more memory variables are associated with each stress state variable (Txx, Tyy, Tzz, Txy, Txz, Tyz). These memory variables contain information about the strain state in the finite-difference system. This information is added to the stress updates defined by EQ. 5d - EQ. 5i and EQ. 6d - EQ. 6i. The memory variables are updated at each timestep. The equations used in this process are defined in the above references.

Currently, E3D uses one relaxation mechanism with 6 memory variables (one for each stress state variable). This means that attenuation is approximated over a frequency range of approximate 1-2 octaves. This is satisfactory for most applications. The medium parameters $Q p$ and $Q s$ are defined at each grid point, as well as a reference frequency $Q f$ where the attenuation will be most accurate. 
Typical $Q$ values might be $10-100$ of soft sediments, and $100-10,000$ for rock. $Q$ is most often considered to be independent of frequency. In sedimentary basins, where the effect of attenuation is most pronounced, the quality factor $Q$ is often defined as a function of the shear velocity. For example, a typical representation is given by

$$
\begin{gathered}
Q s=20 \cdot V s, V_{s}<1.5 \\
Q s=100 \cdot V s, V_{s} \geq 1.5
\end{gathered}
$$

when $V s$ is given in $\mathrm{km} / \mathrm{s}$, and

$$
Q p=1.5 \cdot Q s
$$

Attenuation becomes important for problems with low ground velocities, such as sedimentary basins. This is especially true at frequencies greater than 0.5 to $1.0 \mathrm{hz}$. Attenuation is often not important for regional seismic problems in rock.

Viscoelastic numerical modeling with $Q$ is often significantly more memory and CPU intensive than purely elastic modeling. Instead of 12 variables per grid point as is the case with elastic modeling, finite-difference simulations with attenuation (one relaxation mechanism) require 22 variables per grid point ( 12 elastic variables, 6 memory variables, 4 variables containing the medium parameters defining $Q$ ). Depending on the system and how attenuation is implement, CPU time may be $25-50 \%$ longer.

\subsubsection{Anisotropy}

Seismic wave propagation can be either isotropic or anisotropic. Isotropic propagation implies that the propagation parameters are the same in all directions. For example, at any given homogeneous subregion in the model, the wave propagation velocity is the same in all directions. Anisotropic propagation implies that the propagation parameters are or can be direction dependent. For example, the shear velocity in the horizontal direction may be different than the shear velocity in the vertical direction. Anisotropic modeling is important for accurately modeling subtle features of wave propagation, and is particularly useful in highly fractured rock where cracks and voids may be partially or completely filled by water or other material.

Anisotropy is generally not important for hazard related problems such as those in the NEVADA simulation environment. In addition, anisotropic simulation using E3D is a current development issue. For these reasons, anisotropic propagation will not be discussed here.

\subsection{Boundary conditions}

Seismic energy numerically propagates through the E3D finite-difference grid. However, this grid has finite dimensions and so the seismic energy that reaches the grid boundaries 
is reflected back into the grid domain. This is undesirable because this reflected energy can be misinterpreted as real signal. Methods must be utilized to minimize or eliminate this reflected energy. The simplest method is to increase the grid size, but this is usually impractical because of limited computational resources. Another approach is to use boundary conditions that absorb or damp the seismic energy as it approaches the grid boundaries. The most common method in E3D is paraxial boundary conditions. Paraxial, damping, or attenuation boundary conditions can be used simultaneously. Another issue is the top boundary of the grid. Usually, this is aligned with the earth's surface and hence boundary conditions must be utilized that physically match this free-surface.

\subsubsection{Surface conditions}

For most earth propagation problems, E3D uses free-surface conditions for the top boundary of the grid. These boundary conditions are that the vertical-component stresses ( $T z z$, $T x z, T y z)$ are zero at $z=0$. Algorithmically, this can be implemented by defining these stress terms as odd functions centered at the top boundary. That is, $T z z$ is at the top boundary and can be set to 0 ; the point above the boundary is equal to the reflective image of the point below the boundary.

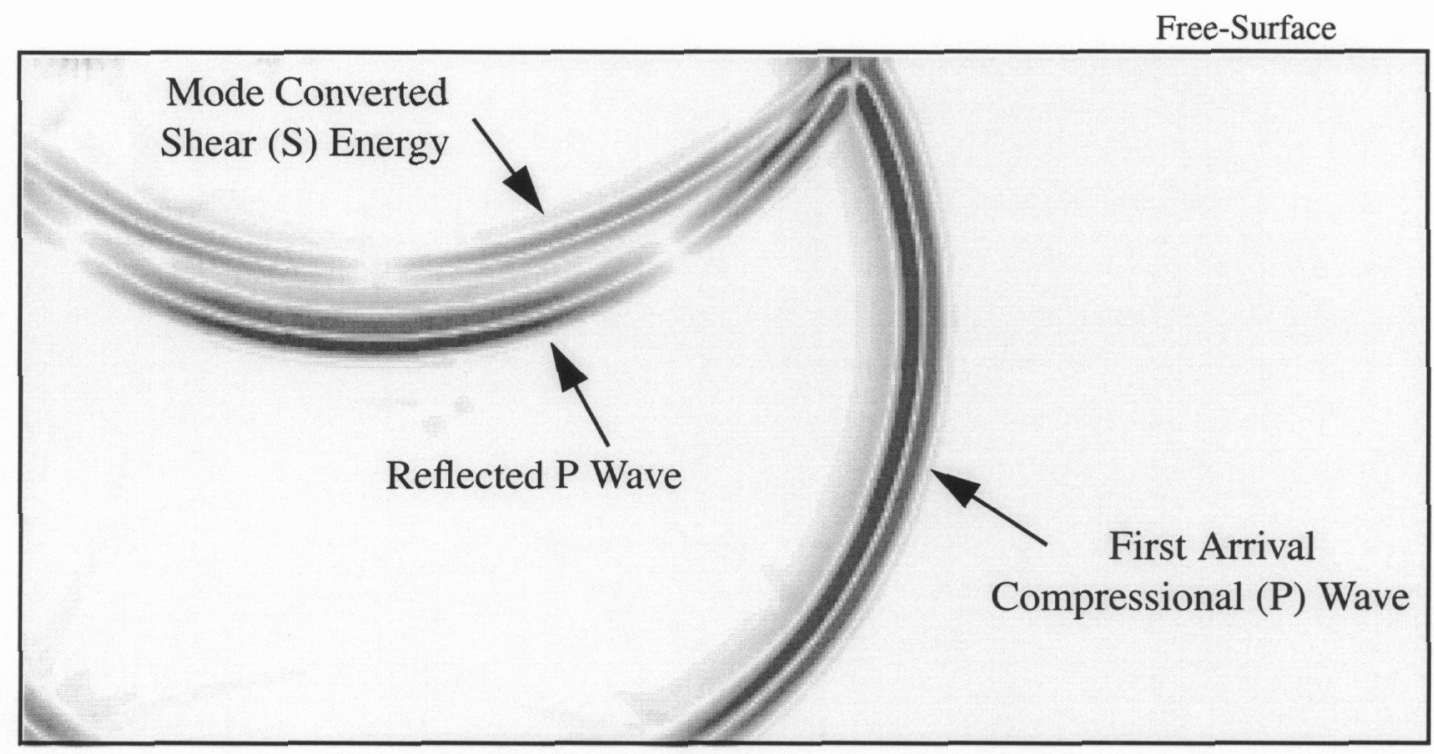

FIGURE 8. Free-surface condition reflects and mode converts seismic energy.

These surface conditions allow seismic energy to be reflected back into the grid domain (Figure 8). This reflection is desirable because it correctly matches the physical conditions at the surface of the real earth. It should be mentioned that energy is converted along the surface. That is, compressional energy is partially converted to shear energy, and shear energy is partially converted to compressional energy. Also, these boundary conditions support the propagation of surface waves (Rayleigh and Love) along the top boundary. 
An alternate mechanism for implementing boundary conditions at the surface is to incorporate an air medium layer above the surface. Although theoretically acceptable, this method generally is not used because the acoustic propagation velocity in air is usually less than the seismic propagation velocity in the earth and hence a finer grid sampling would be required. In addition, the air density is significantly less than the ground density, which can cause undesirable numerical instabilities.

\subsubsection{Paraxial side boundaries}

Paraxial boundary conditions are used to absorb the seismic energy as it reaches the side and bottom boundaries of the grid [Clayton and Engquist, 1977] (Figure 9). As implemented by E3D, the wavefield is assumed to be propagating normal to each boundary. Hence, the wavefield can be extrapolated outward by a one way operator.

As an example, for waves propagating in the positive $x$ direction and striking the right boundary of the grid, the conditions along the boundary must satisfy

$$
\begin{aligned}
& \frac{\partial V_{x}}{\partial x}+1 / V_{p} \cdot \frac{\partial V_{x}}{\partial t}=0 \\
& \frac{\partial V^{y}}{\partial x}+1 / V_{s} \cdot \frac{\partial V_{y}}{\partial t}=0
\end{aligned}
$$

and

$$
\frac{\partial V_{z}}{\partial x}+1 / V_{s} \cdot \frac{\partial V_{z}}{\partial t}=0
$$

These equations are fairly easy to implement and discretize at the side and bottom grid boundaries.

While these paraxial conditions absorb $100 \%$ of the energy that propagates normal to the boundary, they absorb less than $100 \%$ of the energy that propagates at a non-normal angle to the boundary. Hence, energy is still reflected back into the grid. In general, more energy is reflected for greater incidence angle deviations from normal. However, energy that is nearly parallel to one boundary will often reflect off that boundary and hit another boundary at a more perpendicular angle. Nonetheless, artificially reflected boundary energy is almost always of at least some concern in E3D simulations.

\subsubsection{Other boundary conditions}

E3D uses other techniques to minimize artificial boundary reflections. Two common techniques are sponge or damping conditions and intrinsic attenuation using $Q$ values. These techniques can be used either separately or jointly with the paraxial technique. 


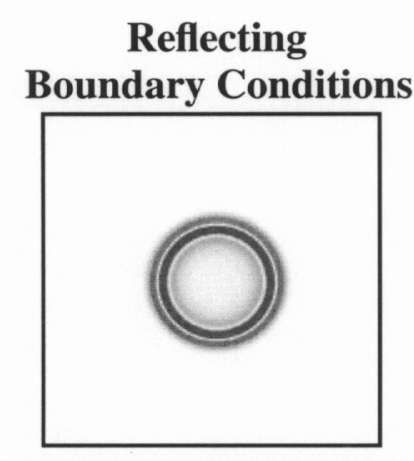

Paraxial Absorbing Boundary Conditions
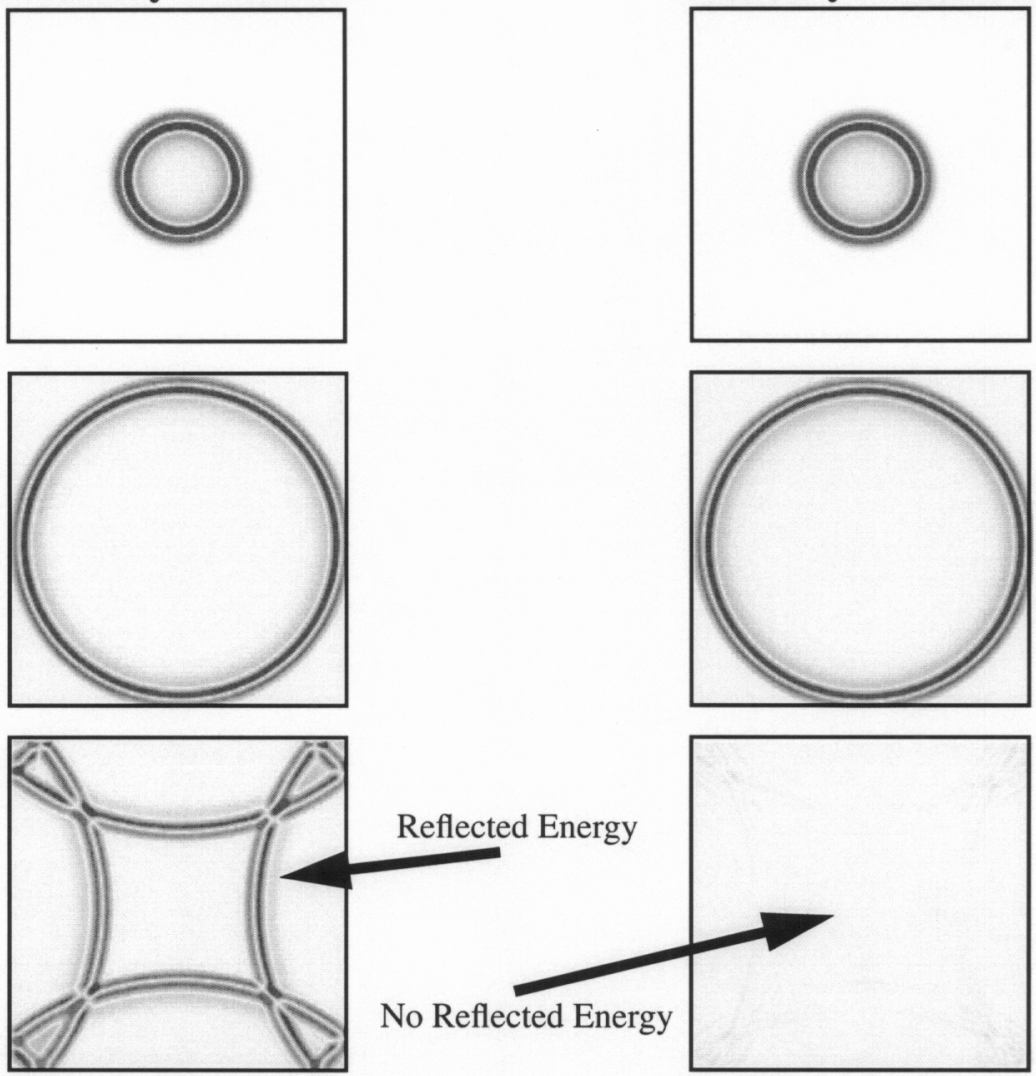

Reflected Energy
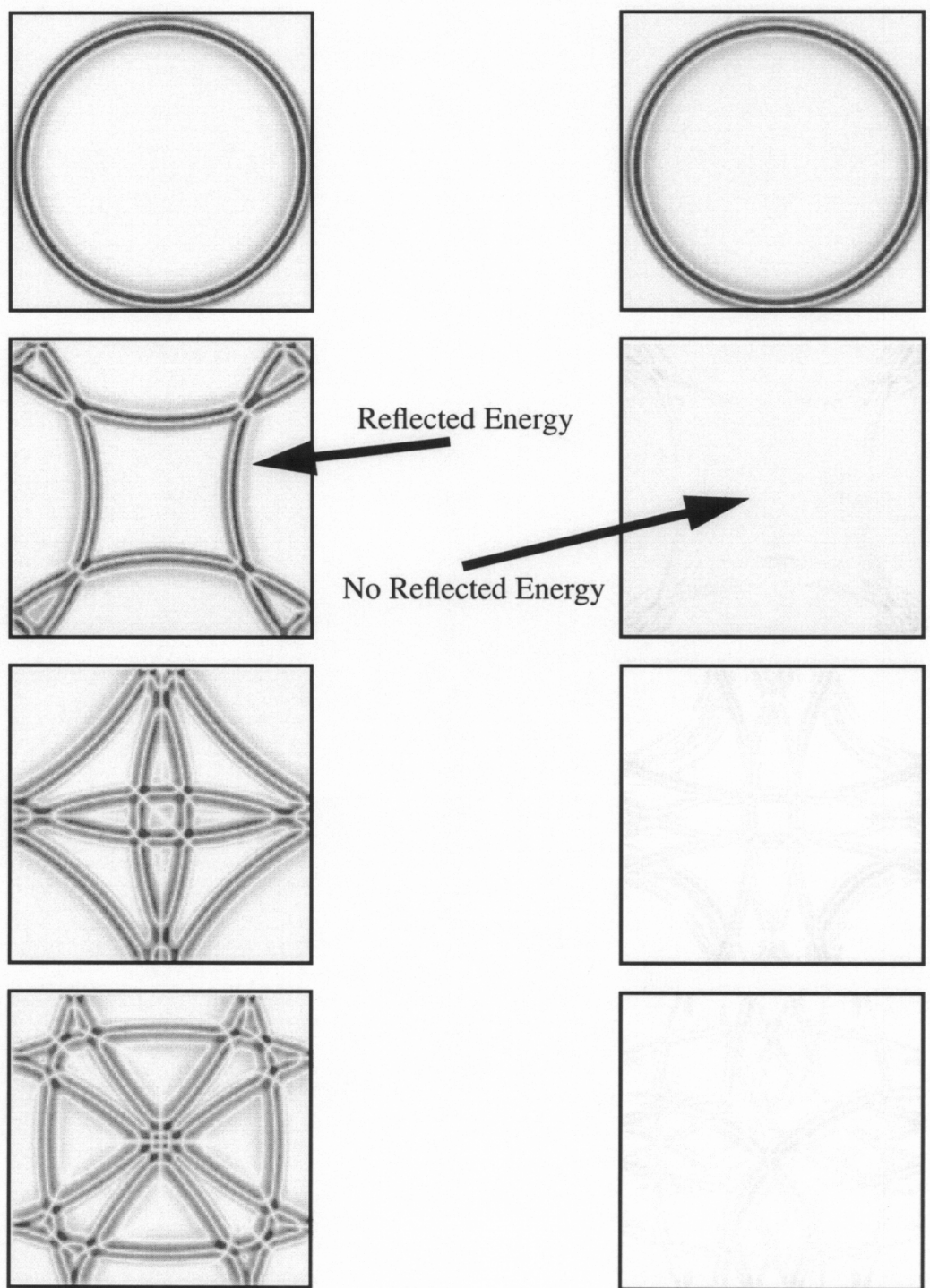

FIGURE 9. Paraxial absorbing boundary conditions prevent energy from being reflected back into grid 
The damping technique [e.g., Cerjan et al., 1988] is a simple reduction in wave amplitude as it approaches the boundary. A "sponge" zone of $N$ grid points is placed around the boundary. At each point, the amplitude of the velocity state variables $(V x, V y, V z)$ is reduced by a small factor. That is,

$$
V=V \cdot(1-(n / N \cdot(1-f)))
$$

where $n$ is the number of grid points from the beginning of the damping zone, and $f$ is a damping factor from 0 . to 1 . In practice, $N$ is usually 20-50 grid points and $f$ is between 0.95 and 1.00. An advantage of the damping technique is it's simplicity. The disadvantage is that the seismic energy isn't really absorbed. Instead, it is reflected back into the grid. This reflection is distributed over a fairly wide zone so it's impact is minimized.

Another method to prevent artificial reflections is to put a highly attenuating low $Q$ region in a buffer zone around each boundary. In practice, such zones are typically 20-50 grid points in width and typical $Q$ values are between 2 and 10 . The advantage of this method is that it intrinsically absorbs the wave energy (i.e., no reflection). The disadvantage is that it must be used with attenuation simulations, and hence requires about twice the computational resource.

\subsection{Topography}

E3D simulations can be performed using numerical models that have a topographic upper boundary representing the surface of the earth (Figure 10). This is important because seismic waves can be significantly influenced by large variations in surface relief (e.g., mountains, hills, valleys). Seismic energy can be focused and/or defocused by these topographic features. Topographic simulations can be performed for both 2-D and 3-D problems.

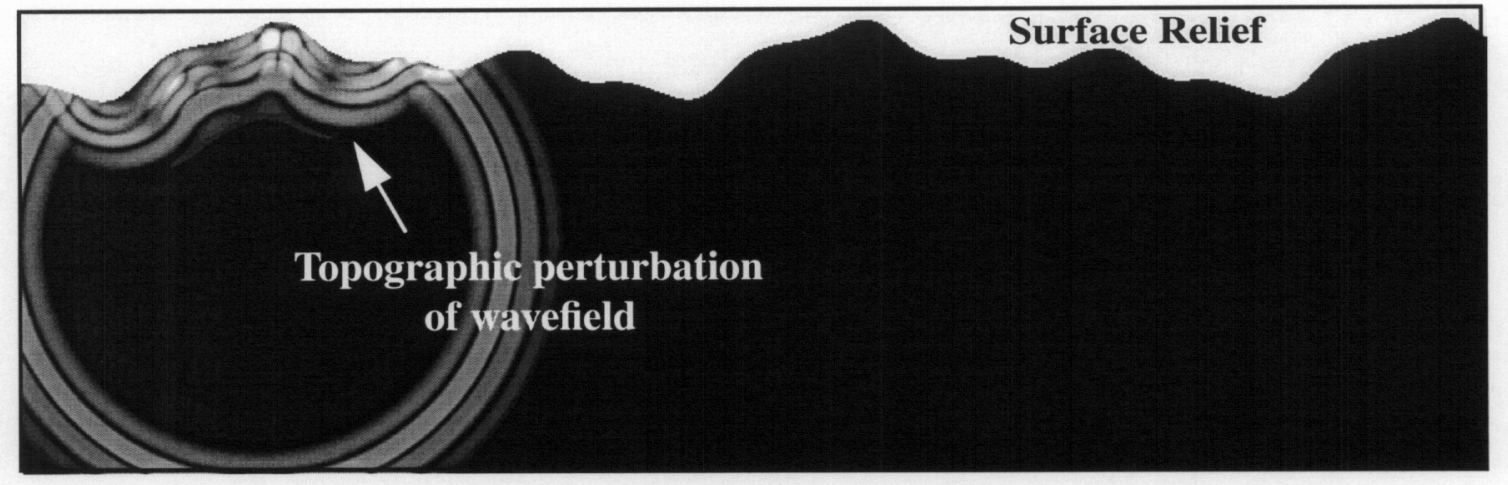

FIGURE 10. The propagation of seismic waves can be influenced by the topography (hills, mountains, valleys) on the surface.

E3D uses a density extinguishing technique to represent a non-horizontal free-surface boundary [e.g., Schultz, 1997]. In this technique, the densities above the surface are exponentially reduced to zero, although numerical considerations require that the densities be 
constrained by a very small minimum value. The medium density at a point above the surface is given by

$$
\rho(z)=\rho(z=0) \cdot R^{n}
$$

where $\rho(z=0)$ is the geologic/medium density at the surface, $R$ is the taper rate, and $n$ is the distance in grid points from the point to the free-surface. A typical value for $R$ is 0.25 . The compressional and shear wave propagation velocities above the surface are constrained to equal the velocities at the surface. Hence, the medium parameters $\mu$ and $\lambda$ are modified as appropriate. The value $n$ is the shortest distance from the point to the surface (in noninteger grid points). A relatively time consuming search algorithm is required to determine this minimum value.

From a users perspective, a grid of points representing the surface boundary is the only topographic-specific input required by E3D. From an algorithmic perspective, the density extinguishing technique is elegant because the grid updates are performed without regard to whether a point is above or below the surface. This method is also highly parallelizable.

However, topographic simulations tend to be more CPU intensive because they require finer grid resolution to produce accurate results. A rule of thumb is that 15 grid points per wavelength are required for topographic simulations. This compares to 10 grid points per wavelength for non topographic simulations. Because of the power law memory and CPU scaling relations, this means that topographic simulations require approximately 3.5 times more memory and 5 times more CPU time than non topographic simulations of the same size. In addition, topographic problems are considerably more difficult to set up and analyze.

\subsection{Source terms}

A seismic source(s) is needed to initiate and drive the propagation of acoustic and/or elastic wave energy that is modeled by E3D. This source is defined by applied force terms $(F x$, $F y$, and $F z$ in EQ. 5a - EQ. 5c and EQ. 6a - EQ. 6c) or by moment rates (Mxx, $M y y, M z z$, $M x y, M x z$, and $M z z$ in EQ. 5d - EQ. 5i and EQ. 6d - EQ. 6i). Two types of seismic sources are considered here: an explosive source and a point double-couple or earthquake source. Both of these can be defined as a function of moment rates. Other types of sources include pure shear, applied force, and distributed slip on a finite-length fault (these are not discussed here). In addition, hybridization is an alternate source mechanism whereby the wavefield computed by a separate finite-difference simulation or another technique can be used to drive the finite-difference model.

\subsubsection{Moment tensor}

The $M x x, M y y, M z z, M x y, M x z$, and $M z z$ terms define the moment tensor, or more precisely the moment rate tensor. These terms are given in units of $d y n e-\mathrm{cm} / \mathrm{s}$ (or $N-\mathrm{m} / \mathrm{s}$ ), and are frequently used in seismology. A seismic source is often defined by symmetric moment 
tensor, and is given by $M_{0}(t) * M$, where $M_{0}(t)$ is a time-dependent scaling or amplitude factor and $M$ is the moment tensor.

The time-history function, $M_{0}(t)$, is defined to represent a realistic source. For example, it might be defined by a Ricker wavelet

$$
M_{0}(t)=M_{0} \cdot(2 \cdot r-1) \cdot e^{-r}
$$

where $M_{O}$ is the amplitude, and $r$ is a functional factor given by $r=\left(\pi \cdot f \cdot\left(t-t_{0}\right)\right)^{2}$, with $f$ the central frequency of the source and $t_{0}$ is an offset time. Alternatively, a time-history function might be defined by a Gaussian wavelet

$$
M_{0}(t)=M_{0} \cdot e^{-r}
$$

A Ricker wavelet is frequently used in seismic exploration because there is no permanent ground displacement (the integral of the function is zero), whereas a Gaussian wavelet is appropriate for explosive or earthquake problems because the ground is permanently displaced (the integral of the function is proportional to the static ground displacement). There are many other types of source time-history functions.

In E3D, it is important that the source-time function be appropriate for the grid sampling. That is, the frequency content of the time-history function should be such that there are at least 5 to 10 grid points per maximum frequency of the source. Alternatively, the synthetic data can be filtered after they are computed.

\subsubsection{Explosive source}

An explosive source is given by $M_{0} * M$, where $M$ is a diagonal tensor of unit 1 . This type of source produces purely compressional waves, although these waves can be converted to shear energy at medium boundaries and the free-surface.

For nuclear explosions, a series of empirical relations can be used to convert from explosion size (in KT) to moment [Patton and Walter, 1993, 1994; Murphy, 1996]. So for example,

$$
\begin{aligned}
& \log M_{0}=1.12 m b+9.55 \\
& m b=0.89 \log M_{0}-8.53 \\
& m b=0.75 \log W+4.05 \\
& \log W=1.33 m b-5.4
\end{aligned}
$$


where $M_{O}$ is the moment of an explosive source in Newton-m, $m b$ is the body wave magnitude of the event, and $W$ is the source size in $K T$. These are for NTS explosions below the water table. So for example,

TABLE 1.

\begin{tabular}{|ccc|}
\hline $\mathbf{M}_{\mathbf{0}}$ & $\mathbf{m b}$ & $\mathbf{W}$ \\
\hline $1.0 \mathrm{e} 13 \mathrm{~N}-\mathrm{m}$ & 3.0 & $\sim 40$ tons \\
$1.1 \mathrm{e} 14 \mathrm{~N}-\mathrm{m}$ & 4.0 & $\sim 1 \mathrm{KT}$ \\
$1.4 \mathrm{e} 15 \mathrm{~N}-\mathrm{m}$ & 5.0 & $\sim 20 \mathrm{KT}$ \\
$1.9 \mathrm{e} 16 \mathrm{~N}-\mathrm{m}$ & 6.0 & $\sim 400 \mathrm{KT}$ \\
\hline
\end{tabular}

A $150 \mathrm{KT}$ explosion is equivalent to a magnitude 5.7 earthquake.

\subsubsection{Earthquake source}

An earthquake point source can be defined by a double couple, which is described by the diagonal and non-diagonal components of a moment tensor. The moment is given by

$$
M_{0}=\mu A D
$$

where $\mu$ is the rigidity, $A$ is the fault area, and $D$ is the displacement of the fault. For finitedifference calculations, a point source operates over a region equal to the grid spacing. Hence, the fault area $A$ is given by the square of the grid spacing.

The moment tensor components can be determined from the strike, dip, and rake of the fault. Using standard seismological notation [Aki and Richards, 1980], the components are given by

$$
\begin{gathered}
M_{x x}=M_{0} \cdot(\sin 1 D \cdot \cos 1 R \cdot \sin 2 S-\sin 2 D \cdot \sin 1 R \cdot \cos 1 S \cdot \cos 1 S) \\
M_{x y}=M_{0} \cdot(\sin 1 D \cdot \cos 1 R \cdot \cos 2 S+0.5 \cdot \sin 2 D \cdot \sin 1 R \cdot \sin 2 S) \\
M_{x z}=-M_{0} \cdot(\cos 1 D \cdot \cos 1 R \cdot \sin 1 S-\cos 2 D \cdot \sin 1 R \cdot \cos 1 S) \\
M_{y y}=-M_{0} \cdot(\sin 1 D \cdot \cos 1 R \cdot \sin 2 S+\sin 2 D \cdot \sin 1 R \cdot \sin 1 S \cdot \sin 1 S) \\
M_{y z}=-M_{0} \cdot(\cos 1 D \cdot \cos 1 R \cdot \cos 1 S+\cos 2 D \cdot \sin 1 R \cdot \sin 1 S) \\
M_{z z}=M_{0} \cdot(\sin 2 D \cdot \sin 1 R)
\end{gathered}
$$

$$
\begin{array}{llr}
\text { where } \quad \sin 1 D=\sin (\text { dip }), & \sin 2 D=\sin (2 \cdot \text { dip }), & \sin 1 S=\sin (\text { strike }), \\
\sin 2 S=\sin (2 \cdot \text { strike }), & \sin 1 R=\sin (\text { rake }), & \cos 1 D=\cos (\text { dip }),
\end{array}
$$


$\cos 2 D=\cos (2 \cdot$ dip $), \quad \cos 1 S=\cos ($ strike $), \quad \cos 2 S=\cos (2 \cdot$ strike $), \quad$ and $\cos 1 R=\cos ($ rake $)$. In this case, strike is the fault direction clockwise from north, dip is measured down from the right of the strike, and rake is the angle between the slip and strike directions, where the slip is the direction of the hanging wall relative to the foot wall (a positive rake is a reverse fault, a negative rake is a normal fault). For example, a strike of 0 ., a dip of 90 ., and a rake of 0 . corresponds to a north-south left-lateral fault or an eastwest right-lateral fault. Note that the above equations use notation where the $\mathrm{x}$ and $\mathrm{y}$ axes are reversed from the notation used in Aki and Richards [1980]. That is, E3D defines x to the east and $y$ to the north.

The moment amplitude $M_{O}$ can be computed from the earthquake magnitude $M_{w}$ by the empirical relation

$$
M_{w}=\log \left(M_{0}\right) / 1.5-10.73
$$

where $M_{0}$ is in dyne-cm. For example, a moment amplitude of $1.24 e 25$ dyne-cm is equivalent to a magnitude 6 earthquake.

\subsubsection{Hybridization}

Hybridization is a mechanism that combines wave propagation (or other) simulations using two different techniques. For example, a computationally inexpensive technique such as reflectivity can be used where the geology is defined by a simple 1-D layered model, while a computationally expensive but more robust technique such as finite-differencing can be used where the geology is more complex and must be defined by a 3-D heterogeneous model. E3D offers a hybridization mechanism to utilize as source input the wave propagation output from other simulation techniques. However, this functionality is not well formulated and it is not used in the NEVADA simulation environment. Hence, it will not be discussed here.

\subsection{Input/Output}

E3D offers several features that facilitate model input and allow simulated or synthetically computed results to be displayed and saved in a variety of data representations. Input features include simple ASCII-text parameter descriptions, ASCII-text parameter representations of the geologic model, gridded binary representations of the geologic model, source time history functions, distributed fault representations, and data compression. Output features include run-time visualization, point ground motion time history at selected points (seismograms), ground motion time history at multiple points (traces), volumetric representation, and image planes of seismic wave propagation.

E3D has restart capabilities, although this feature is nonrobust and is not often used. 


\subsubsection{Input}

The basic input to E3D is an ASCII text file that defines the simulation parameters, geologic model, source function, and output formats. Other files, both ASCII and binary, can be referenced by this input file. Runtime simulation parameters such as grid size, grid spacing, timestep interval, and number of timesteps are defined in this input file.

For 3-D problems, the geologic model can be defined in one of three ways. For simple geologic models, it can be defined in the input file as a set of simple statements. For simple or more complex geologic models, it can be defined as one or more binary input files. The seismic velocity ( $V p$ and $V s$ ), density, and optionally $Q p, Q s$, and $Q f$ can be set at each grid point in the grid (or a subset/superset of the grid). Because the 3-D model is often large, these binary input files can be run-length-encoded compressed. The third method to define the geologic model is via an ASCII text file of a parameterized 3-D model. The parameterization method will not be described here.

One or more seismic sources can be input. The source parameters are defined in the input file, and the source time history function is either preset or input via a binary input file.

\subsubsection{Output}

Synthetic data computed via E3D can be output as time histories at individual points, or as a collection of points in multiplexed trace format. E3D can output all state variables ( $V x$, $V y, V z, T x x, T y y, T z z, T x y, T x z, T y z)$, as well as the compressional and shear potentials. In addition, synthetic data can be output as a set of 2-D gridded time slices that are useful for constructing movies or animations of the seismic waves as they propagate through the geologic model. These 2-D gridded planes can be oriented at an arbitrary position in any of the 3 axis dimensions. The model variables ( $V p, V s$, density) can be output in addition to the state variables. Also, the maximum horizontal velocity and the energy content of a simulation can be obtained. Volumes or data cubes can be output, although this feature is seldom used due to the shear volume of most 3-D models.

A runtime visualization is available with $\mathrm{E} 3 \mathrm{D}$ that allows the user to observe the progression of the wavefield in real time during the simulation. The observables are the compressional and shear potentials. This is particularly useful as a means to observe mode converted energy, as well as a mechanism to debug a model when a problem occurs.

\subsubsection{Receiver and output formulations}

Synthetic data for each state variable (e.g., $V x, V y, V z$ ) can be output at arbitrarily located positions within the finite-difference grid. Where these data do not fall on a grid point, a simple 1st-order interpolation scheme of the surrounding points is used to compute the value at the specified position.

The compressional and shear potentials are often output at selected points or as a gridded file used to construct images. The compressional component $P$ at a given point is computed from the divergence of the velocity wavefield. That is, 


$$
P=\frac{\partial V_{x}}{\partial x}+\frac{\partial V_{y}}{\partial y}+\frac{\partial V_{z}}{\partial z}
$$

Computation of the shear component $S$ is ambiguous, but in essence it is computed from the curl of the velocity wavefield. In the NEVADA simulation environment, it is given by

$$
S=0.5 \cdot\left(\left(\frac{\partial V_{z}}{\partial y}-\frac{\partial V_{y}}{\partial z}\right)^{2}+\left(\frac{\partial V_{x}}{\partial z}-\frac{\partial V_{z}}{\partial x}\right)^{2}+\left(\frac{\partial V_{y}}{\partial x}-\frac{\partial V_{x}}{\partial y}\right)^{2}\right)^{0.5}
$$

\subsection{Computational issues}

E3D has been designed and optimized to simulate large 3-D problems in minimal time. The most significant aspect is parallelization, which allows extremely large models to be simulated using massively parallel computers. In addition, E3D was designed to be selfcontained, making it highly portable to a variety of platforms.

\subsubsection{Platforms}

E3D runs on a variety of computer platforms, from desktop workstations to high performance systems that use more than a thousand CPU's and communicate via a very highspeed communications network. E3D has been successfully ported to a number of workstations (e.g., Sun, HP, and SGI), Linux-based Intel platforms, vector processors (e.g., Fujitsu 7600, Cray C-90), shared memory processors (e.g., SGI Origin, Sun Enterprise, DEC Alpha clusters), and massively parallel processors (e.g., nCube-2, Meiko CS-2, Cray T3D, Cray T3E, IBM SP-2, DEC/Compaq, Sun and Linux clusters). In general, it requires approximately 1 hour to 1 day to port E3D to a new system. However, optimization techniques on a given platform can be much more time intensive.

\subsubsection{Low-level optimization}

E3D is designed to take advantage of cache coherent computer architectures. The computational sections of the code are highly vectorized, which significantly boosts performance even on desktop workstations. The data arrays are sequentially accessed from memory. The core computations consist of floating point additions and multiplies. There are no floating point divisions. E3D is written in ANSI C. However, FORTRAN subroutines are used for the low-level numerical computations that perform the finite-difference stencil operations (with optional $\mathrm{C}$ routines, if FORTRAN is not available or less efficient on a given computer architecture.

\subsubsection{Variable density grid}

Fundamentally, E3D uses a structured regularly spaced grid. This is often inefficient because the grid spacing is a function of the lowest geologic velocity in the earth model, and hence regions with significantly higher wave propagation speeds will be over sampled. This can be significant for many earth models that tend to have low velocities in a 
narrow zone near the surface and much higher velocities for the rest of the model. It is not uncommon to have a factor of 8 disparity between the slowest and fastest regions of a geologic model (e.g., $1 \mathrm{~km} / \mathrm{s}$ near the surface and $8 \mathrm{~km} / \mathrm{s}$ at depth). In this situation, the high velocity regions will be over sampled by a factor of 8 . In a limiting case, this produces a computational inefficiency that is more than 500 times more inefficient than it needs to be.

A variable density grid is one with a grid spacing that is mapped to the geologic velocity. That is, a computationally intensive fine grid is used in regions of low geologic velocity and a computationally efficient coarse grid is used in regions of high geologic velocity. Because the high velocity regions tend to dominate the model, a variable density grid can result in a considerable savings of computer resources. For typical problems, it is not unrealistic for a variable density grid to be 10 or more times more efficient than a uniformly spaced grid.

E3D features a rudimentary variable density grid structure. Sections of the grid can be segmented in computational cubes, with each cube having a grid spacing appropriate for the geology. In practice, the grid spacing from one cube to the next will vary by a factor of two. Each computational cube communicates with it's neighbors via interpolation. The variable density grid structure in E3D is not robust and it is not parallelized. Currently, it is not part of the NEVADA simulation environment.

\subsubsection{Propagating grids}

E3D features four propagating grid mechanisms. In the first, which is most important for the NEVADA simulation environment, regions of the finite-difference grid not yet activated by seismic energy are void of numerical computations. This mechanism saves CPU resources, especially in the early stages of a simulation. In some situations, it is possible to decrease the simulation time by a factor of two by using this propagating grid. This feature is less efficient in parallel applications, depending on how the computational domain is distributed over the parallel computer system.

The second propagating grid mechanism is similar to the first, except that regions of the grid that will not contribute to synthetic data collected along the grid surface also will be void of numerical computation. This reduces CPU time near the end of a simulation. The third mechanism allows the user to specify a propagating region that travels at a certain velocity. It is used to track a specific phase and is most useful in regional simulations of wave propagation. The fourth mechanism allows the user to computationally activate regions of a grid as a function of time. It is the most robust mechanism, but also the most difficult to formulate.

\subsubsection{Parallelization}

E3D is parallelized to run on massively parallel computer systems. The explicit finite-difference formulation that is discretized on a regular numerical grid is well suited for parallelization. The numerical grid is spatially decomposed over the individual processes activated in a parallel run-time environment. Each decomposed subgrid communicates with it's neighbors using MPI or an equivalent message passing interface. The 4th-order 
differencing scheme requires that each subgrid have two rows of boundary or ghost zones that virtually overlap it's neighbors. Communication occurs twice every timestep: once after the velocity updates and once after the stress updates.

\subsubsection{Performance}

The observed single CPU performance of the core computational elements of E3D are a significant fraction of the peak CPU performance. On a Fujitsu 7600 vector processor, for example, a sustained performance of $57 \%$ peak processor speed was observed. On most architectures, a sustained performance of $25-40 \%$ peak speed is observed. Two-dimensional simulations tend to be faster (more efficient) than three-dimensional simulations. Performance can be significantly reduced in conjunction with intensive $\mathrm{I} / \mathrm{O}$, run-time visualization, and certain boundary conditions.

The performance on massively parallel processors is equally impressive. Although E3D is not yet optimally configured for parallel processing, it is not uncommon to achieve sustained speeds of $25-30 \%$ peak on these systems. E3D tends to scale well with problem size. That is, a large problem using many CPU's is as efficient as a problem half the size using half the number of CPU's. However, a small problem using many CPU's may not be very efficient.

E3D tends to be memory limited. That is, problem size is determined by the available computer memory. However, since simulation time scales at a faster rate than memory utilization, larger problems are beginning to become CPU limited.

\subsection{References}

Aki, K., and P. G. Richards, Quantitative seismology: theory and methods, W. H. Freeman and Company, 932 p, 1980.

Blanch, J. O., J. O. A. Robertsson, and W. W. Symes, Modeling of a constant Q: Methodology and algorithm for an efficient and optimally inexpensive viscoelastic technique, Geophysics, 60, 176-184, 1995.

Cerjan, C., D. Kosloff, R. Kosloff, and M. Reshef, A nonreflecting boundary condition for discrete acoustic and elastic wave equations, Geophysics, 50, 705-708, 1988.

Clayton, R., and B. Engquist, Absorbing boundary conditions for acoustic and elastic wave equations, Bull. Seism. Soc. Am., 67, 1529-1540, 1977.

Levander, A. R., Fourth-order finite-difference P-SV seismograms, Geophysics, 53, 14251436, 1988.

Madariaga, R., Dynamics of an expanding circular fault, Bull. Seism. Soc. Am., 66, 639$666,1976$. 
Robertsson, J. O. A., J. O. Blanch, and W. W. Symes, Viscoelastic finite-difference modeling, Geophysics, 59, 1444-1456, 1994.

Schultz, C. A., A density-tapering approach for modeling the seismic response of free-surface topography, Geophys. Res. Lett., 24, 2809-2812, 1997.

Virieux, J., P-SV wave propagation in heterogeneous media: velocity-stress finite-difference method, Geophysics, 51, 889-901. 


\subsection{Structural response modeling}

The ultimate goal of seismic hazard analyses is to quantify the risk associated with subjecting major structures to seismic ground motions. The second principal component of the NEVADA simulation environment is a finite element based program for simulating the linear and nonlinear response of structures. The structural response component of NEVADA is based on a finite deformation model which explicitly tracks the displacement of the structure through time. The model also incorporates elasto-plastic material behavior for the structural elements and utilizes path-independent integration for update of the member forces. The time stepping solution for the equation of motion employs a classical Newmark-Beta algorithm with Newton-Raphson equilibrium iterations within each time step.

The structural model was developed to allow efficient linear and nonlinear analyses of building structures within the same solution framework. This approach was followed so that the application space of the NEVADA framework would span from nuclear event evaluations, where the structural response is expected to be well within the linear range, to large natural earthquakes where the response of structures would typically enter the nonlinear range due to physical nonlinearities (material damage) and geometric nonlinearities in the structure (large lateral displacements in a tall building). The structural model also has robust nonlinear static analyses capabilities to allow static push-over computations for building structures. The application space of the structural model is shown schematically in Figure 11.

Simulating weak-motion nuclear events

Simulating strong-motion earthquakes
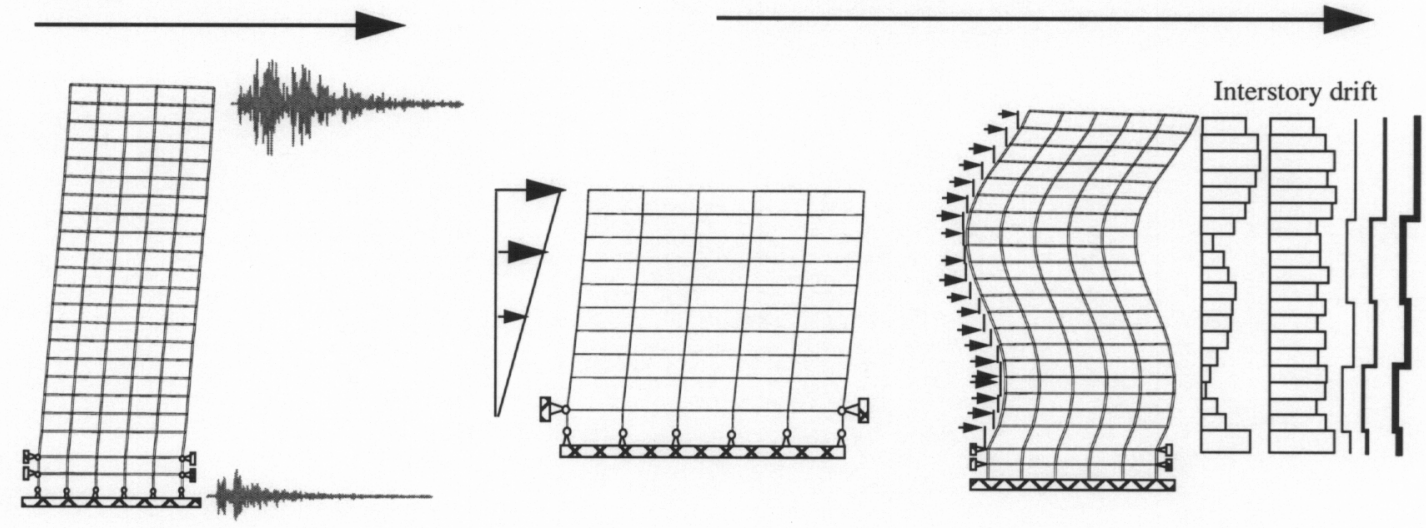

Linear dynamic analysis

1) Eigenvalue solution for natural modeshapes

2) Direct integration of equations of motion with infinitesimal deformation and linear material

\section{Nonlinear static analysis}

1) Push-over analysis with finite deformation and elastoplastic materials. Incremental loading with NewtonRaphson equilibrium iterations

\section{Nonlinear dynamic analysis}

1) Time-stepping nonlinear analysis with finite deformation and elasto-plastic material response. Newton equilibrium iterations within a time step

FIGURE 11. Application space and capabilities in the structural simulation model. 
The details of the structural model solution algorithms are summarized in this section.

\subsection{Global nonlinear solution algorithms}

Transient seismic analysis of building structures requires efficient time stepping algorithms which are capable of solving the equations of motion over extended time periods. For example, a large earthquake may induce 30-60 seconds or more of strong structural vibration. In cases where the structure remains in the linear regime, time history solutions are most economically obtained by exploiting the orthogonality properties of the natural modes of the structure and transforming to normal mode equations. As a result of the fact that a relatively few modes can capture the response of typical building structures, the modal transformation effectively results in a significant reduction of degrees of freedom. Modal equations of buildings subjected to piecewise linear ground acceleration time histories (i.e. time histories created by "connecting the dots" of digital ground motion records) are readily and accurately solved with closed-form analytical solutions for piecewise linear forcing functions.

Typically designed buildings are expected to incur some level of damage, with the potential for corresponding nonlinear response behavior, during severe earthquakes. In order to understand and predict the full response of buildings subjected to extreme earthquake motions, it is desirable to develop nonlinear computational models capable of simulating the response into the nonlinear range. Amplitude dependent nonlinear equations of motion, where the nonlinearities arise from either large displacements, material nonlinearities, or both, must be solved with direct time history integration schemes. Because of the relatively long duration time scales associated with earthquakes, explicit time integration schemes prove prohibitively expensive for structures because of the time step size requirements imposed by Courant stability considerations. Unconditionally stable implicit time integration schemes are generally significantly more economical and are typically employed in nonlinear time history simulations for earthquake loading. The implicit integration solution framework applied to the building equations of motion and employed in the nonlinear building simulation program NEVADA is described in this section.

\subsection{Static solution framework}

For purposes of discussion of the solution algorithms, reference will be made to a discretized finite element model of a building structure. In general, the building structural model may include many different element types in order to represent frames, floor slabs, shear walls, curtain walls etc. (Figure 12). Regardless of the element types included, the global variables of interest include the nodal displacements and rotations, denoted by the global displacement vector $\{D\} \in R^{m}$, the externally applied nodal load vector $\{P\} \in R^{m}$ and the internal resisting force vector $\{Q\} \in R^{m}$ where $R^{m}$ denotes the vector space of dimension "m" (Figure 13). 

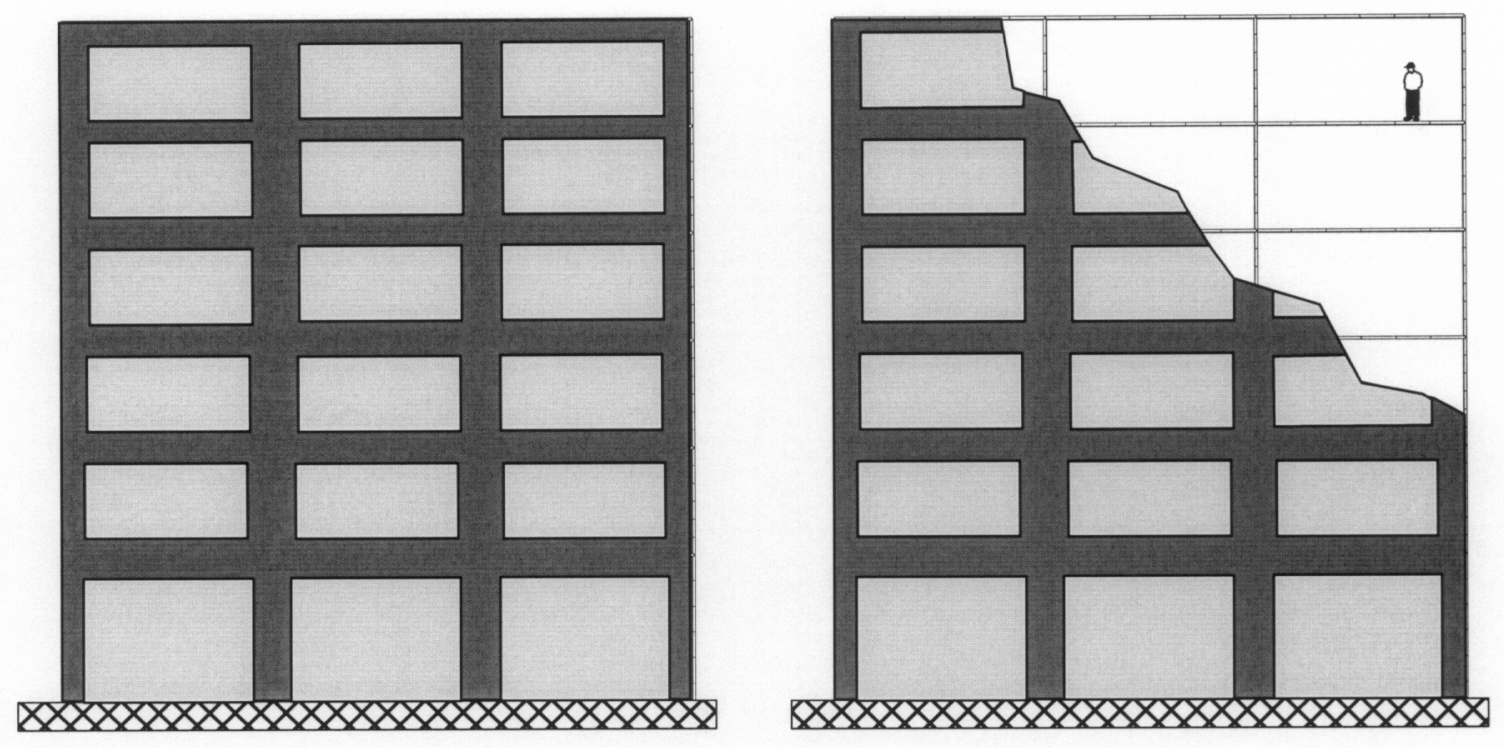

a)

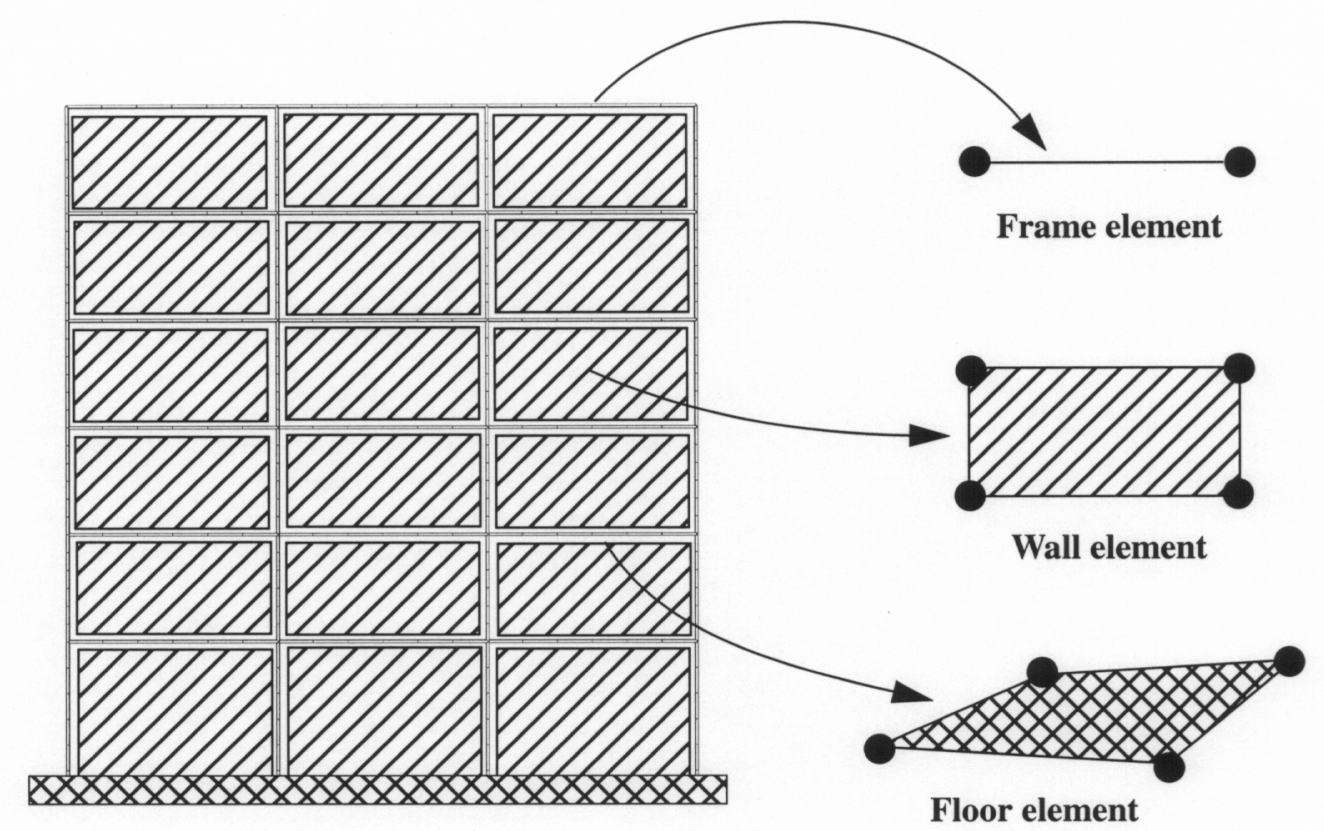

b)

FIGURE 12. Components of a building and computational building model. a) Building structure; b) building numerical model.

\subsubsection{Linear response analysis}

The static equilibrium of a structure responding in the linear regime can be stated in terms of a balance of externally applied loads and internal resisting forces in the structure, i.e. 


$$
\{Q\{D\}\}-\{P\}=0
$$

Where $\{Q\{D\}\}$ is the vector of internal resisting forces and $\{P\}$ is the vector of applied external forces (see Figure 13).

For a structure responding to static loading in the linear response regime, the internal resisting forces of the structure $\{Q(D)\}$ in global coordinate directions are linear functions of the structural displacements $\{D\}$, which can be expressed,

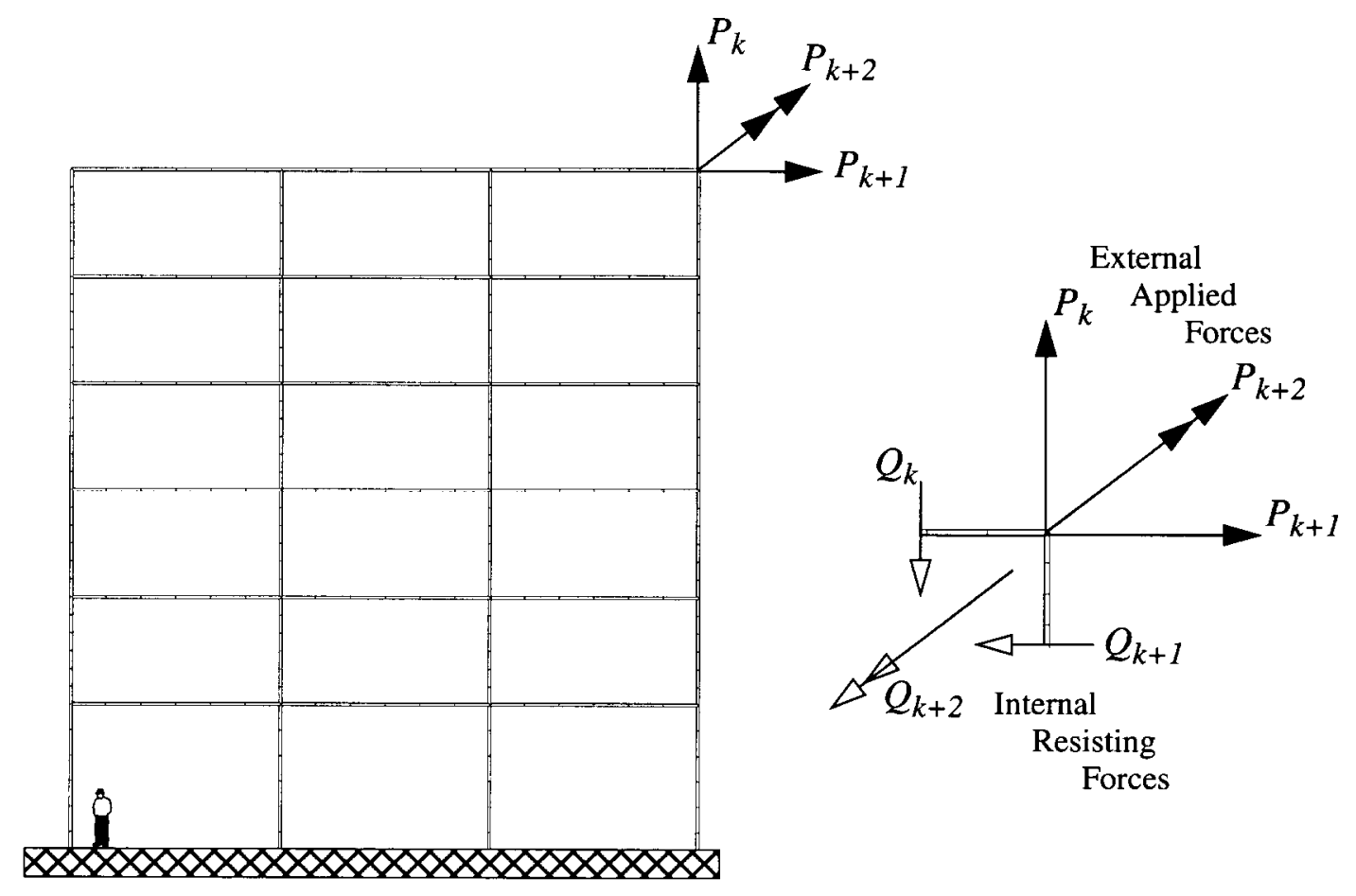

FIGURE 13. External and internal forces in global coordinates.

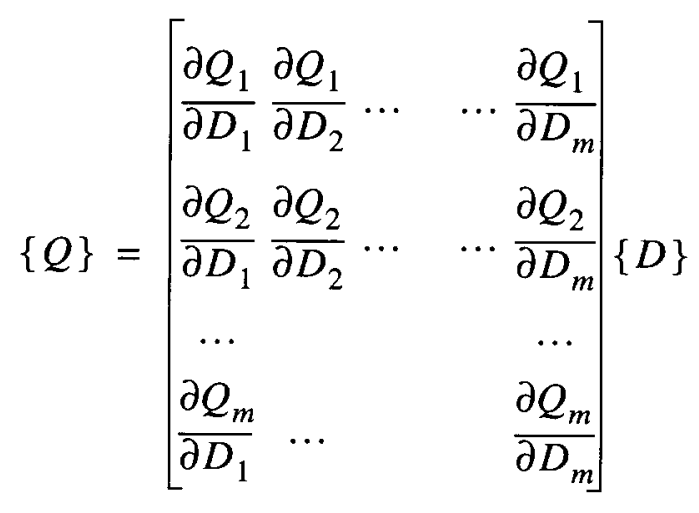


where the stiffness coefficients $\frac{\partial Q_{i}}{\partial D_{j}}$ are constants and represent the rate of change of internal force "i" with respect to displacement " $j$ ". The matrix of EQ. 23 is the classical stiffness matrix of the elastic system and EQ. 23 can be written

$$
\{Q\}=[K]\{D\}
$$

Thus the equilibrium equations expressed in EQ. 22 can be written

$$
[K]\{D\}=\{P\}
$$

and the solution of this system of equations for a given load vector $\{P\}$ provides the total system displacement vector $\{D\}$. The one-step solution procedure for the linear system is indicated schematically in Figure 14.

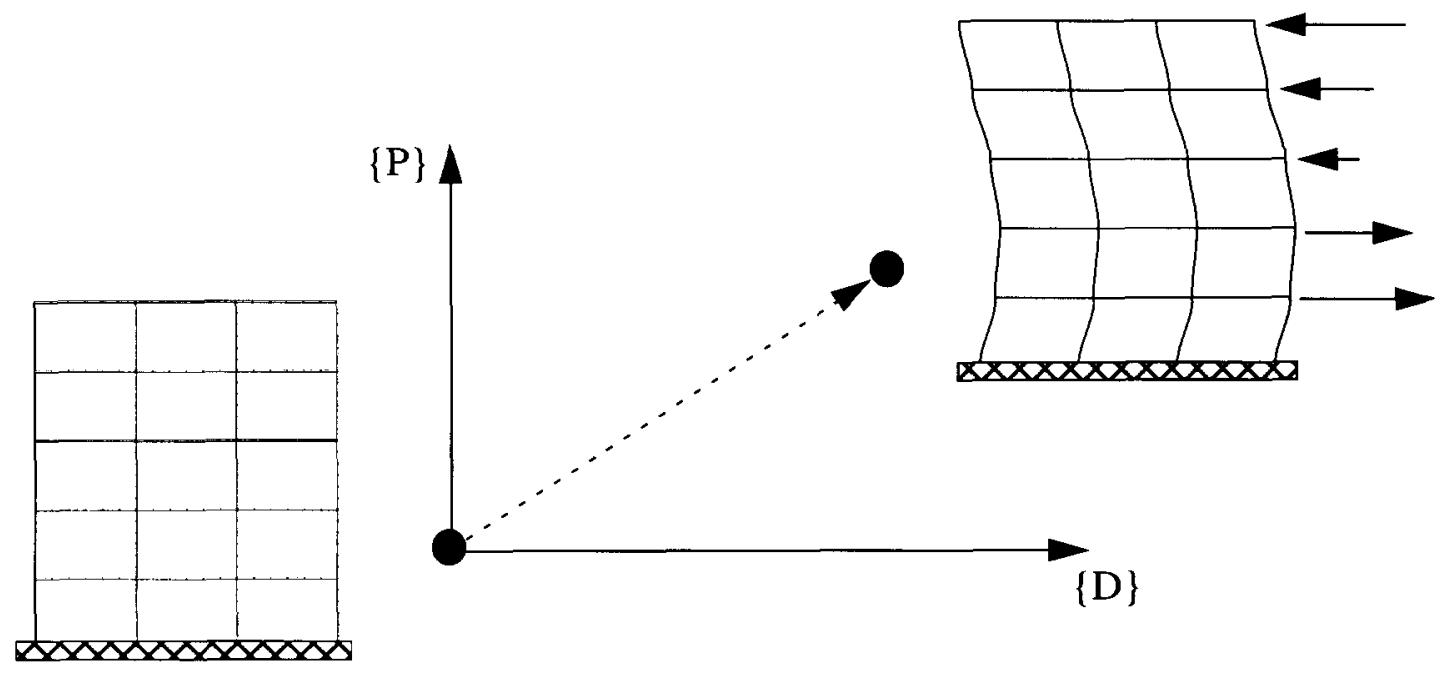

FIGURE 14. One-step solution of linear system model.

\subsubsection{Nonlinear response analysis}

For the case in which a building structure responds in the nonlinear regime, the internal resisting forces of the structure are no longer linear functions of the structure displacments. Consequently, the global equations of equilibrium are nonlinear, precluding a direct one-step solution. The nonlinear character of the system model necessitates an incremental or iterative solution of the equilibrium equations. If the computational model residual vector is defined as the difference between the external applied forces and the internal resisting forces, the residual can be written,

$$
R\{D\}=Q\{D\}-\{P\}
$$


where $Q\{D\}$ is now a nonlinear function of displacements as a result of large displacement induced geometric nonlinearities or material nonlinear behavior. Then a displaced shape at an equilibrium configuration of the structure, denoted $\left\{D^{\text {h }}\right\}$, would result in a null residual vector, i.e.

$$
\left\{R\left\{D^{\not 3}\right\}\right\}=\{0\}
$$

If $\{D\}_{j}$ is the jth approximation of $\left\{D^{\hbar}\right\}$, and $\{D\}_{j}$ is assumed "near" the equilibrium configuration, then a Taylor series expansion of the residual vector about $\{D\}_{j}$ yields,

$$
\left\{R\left\{D^{\text {㟧 }}\right\}\right\}=\left\{R\{D\}_{j}\right\}+\left[\frac{\partial}{\partial D}\left\{R\{D\}_{j}\right\}\right]\{\Delta D\}_{j}+o(\Delta D)^{2}
$$

Where,

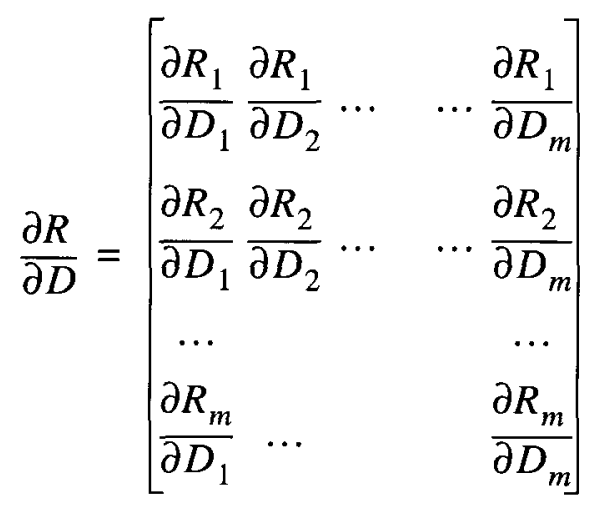

and from EQ. 26 then,

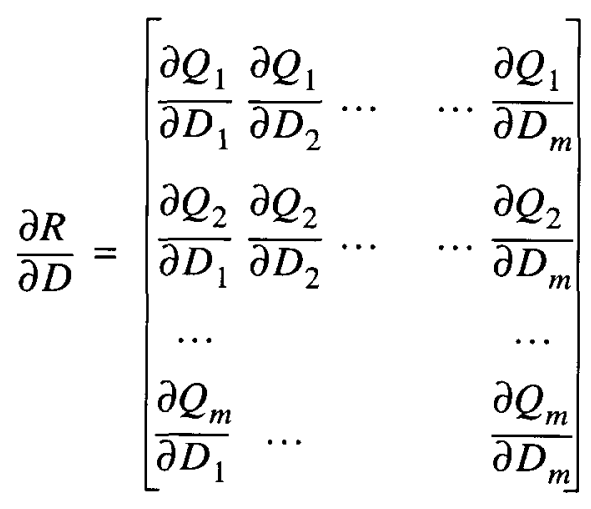


Neglecting higher order terms in EQ. 28, and invoking the fact that the residual is zero at the equilibrium state (i.e $\left\{R\left\{D^{\hbar 3}\right\}\right\}=\{0\}$ yields,

$$
\left[\frac{\partial}{\partial D}\left\{R\{D\}_{j}\right\}\right]\{\Delta D\}_{j}=\left\{R\{D\}_{j}\right\}
$$

For the nonlinear system the stiffness coefficients in EQ. 31 are no longer constant but vary with amplitude, the instantaneous (or tangent) stiffness, denoted $\left[K_{I}\{D\}\right]$ is defined as the amplitude dependent rate of change of the internal resisting forces with displacement, and

$$
\left[K_{I}\{D\}_{j}\right] \equiv\left[\frac{\partial}{\partial D}\left\{Q\{D\}_{j}\right\}\right]
$$

and EQ. 32 can be written

$$
\left[K_{I}\{D\}_{j}\right]\{\Delta D\}_{j}=-\left\{R\{D\}_{j}\right\}
$$

or

$$
\left[K_{I}\{D\}_{j}\right]\{\Delta D\}_{j}=\{P\}-\left\{Q\{D\}_{j}\right\}
$$

and the displacement update after each iteration is then

$$
\{D\}_{j+1}=\{D\}_{j}+\{\Delta D\}_{j}
$$

EQ. 34 provides the basis for a Newton-Raphson solution algorithm in which the externally applied loads are applied incrementally and equilibrium iterations are performed within each load increment to drive the equilibrium residual to an acceptably small value. For systems with strong nonlinear behavior, it is typically necessary to not only perform equilibrium iterations within a load increment, but to also divide the overal load into a number of increments, i.e. a one-step solution will often not converge even with equilibrium iterations when strong nonlinearities are present. It may also be necessary to perform load incrementation to adequately track path dependency when nonlinear material behavior is present. Letting " $n$ " denote the nth load increment, the equation for incremental displacements can be written,

$$
\left[K_{I}\{D\}_{j}^{n}\right]\{\Delta D\}_{j}=\{P\}^{n}-\left\{Q\{D\}_{j}^{n}\right\}
$$

and

$$
\{D\}_{j+1}^{n}=\{D\}_{j}^{n}+\{\Delta D\}_{j}
$$


An incremental and iterative solution procedure is shown schematically in Figure 15.

\subsubsection{Linear time-history analysis}

The fundamental problem in transient dynamic analyses is establishing a procedure for efficiently stepping from time " $n-1$ " to time " $n$ " as indicated in Figure 16. Applying Newton's law to the building system model at time $n$ yields the coupled dynamic equations of motion of the system model,

$$
[M]\{\ddot{D}\}^{n}+[C]\{\dot{D}\}^{n}+[K]\{D\}^{n}=\{P\}^{n}
$$

where

$[M]\{\ddot{D}\}^{n}=$ the vector of nodal inertial forces, $([M]=$ system mass matrix $)$

$[C]\{\dot{D}\}^{n}=$ the vector of nodal damping forces, $([C]=$ system damping matrix $)$

$[K]\{D\}^{n}=$ the vector of internal resisting forces, $([K]=$ system stiffness matrix $)$

$\{P\}^{n}=$ the vector of applied external loads

For the NONLINBLDG program, classical Newmark formulas are utilized to arrive at an unconditionally stable implicit solution algorithm (Newmark, 1959). The Newmark for-

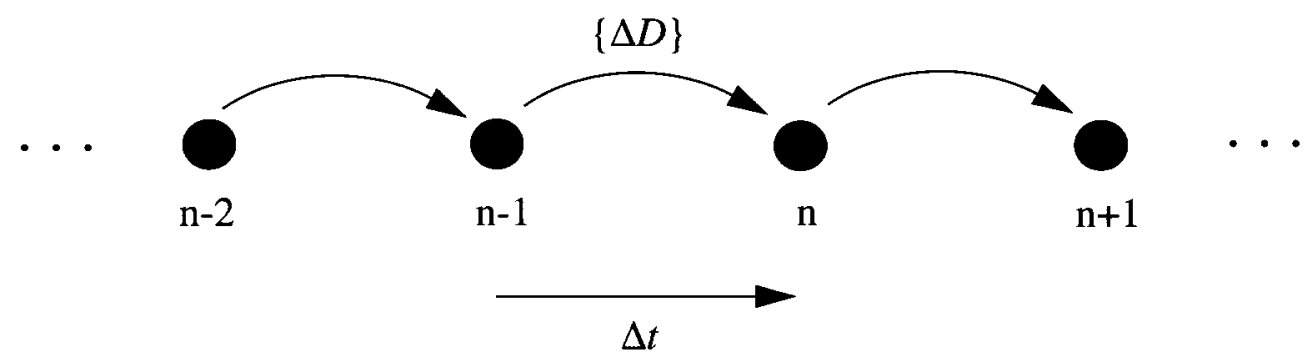

FIGURE 16. Time stepping increments.

mulas provide relationships for the forward displacement and velocity,

$$
\begin{gathered}
\{D\}^{n}=\{D\}^{n-1}+\Delta t\{\dot{D}\}^{n-1}+\frac{\Delta t^{2}}{2}(1-2 \beta)\{\ddot{D}\}^{n-1}+\Delta t^{2} \beta\{\ddot{D}\}^{n} \\
\{\dot{D}\}^{n}=\{\dot{D}\}^{n-1}+\Delta t(1-\gamma)\{\ddot{D}\}^{n-1}+\Delta t \gamma\{\ddot{D}\}^{n}
\end{gathered}
$$




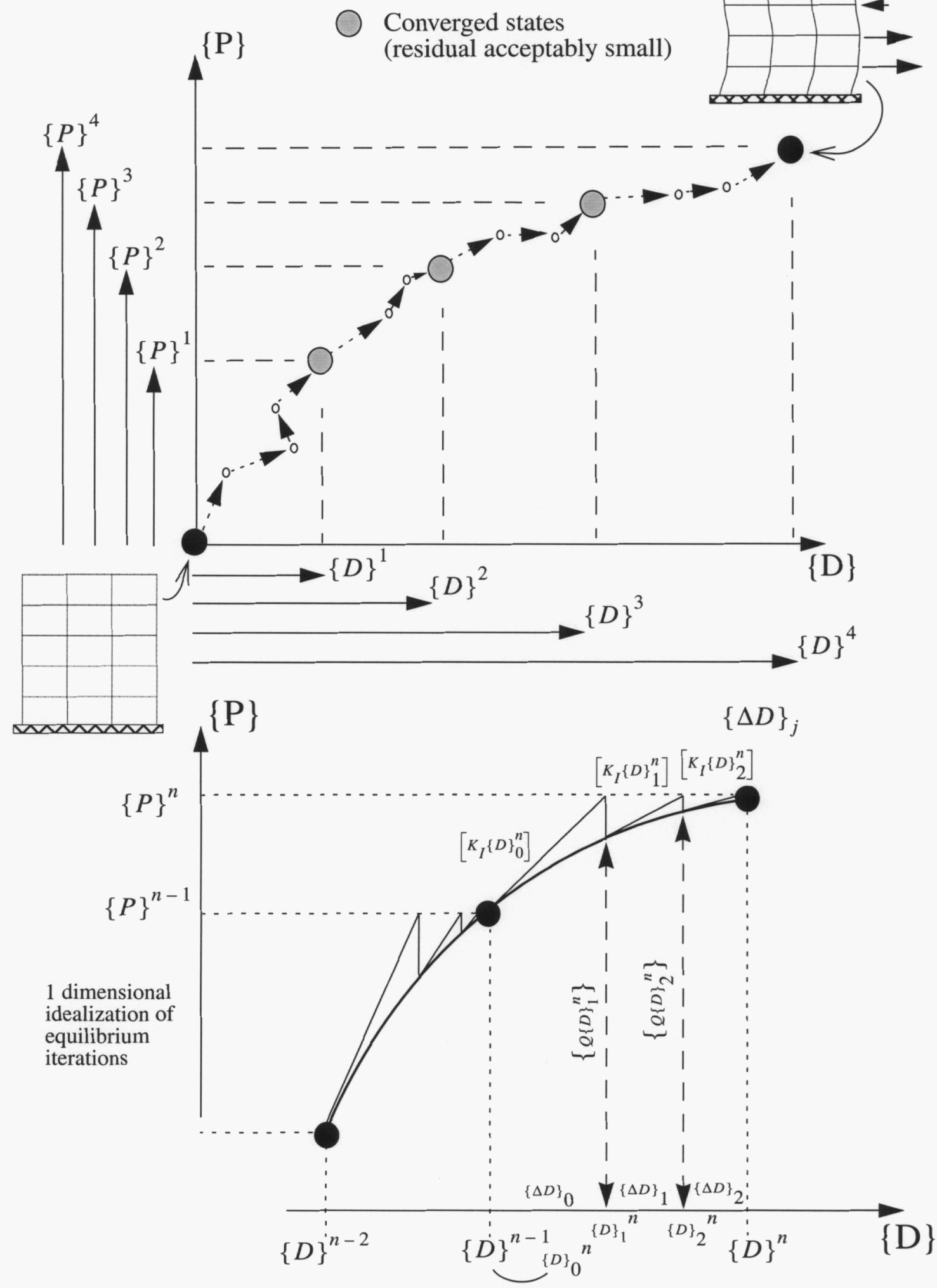

FIGURE 15. Incremental-iterative solution of a nonlinear system. 
where $\gamma$ and $\beta$ are constants which can be chosen to control stability and accuracy of the integration scheme $\left(\gamma=\frac{1}{2}\right.$ and $\beta=\frac{1}{4}$ correspond to utilizing an average acceleration across a time step). Solving EQ. 40 for the forward acceleration yields

$$
\{\ddot{D}\}^{n}=\frac{1}{\Delta t^{2} \beta}\{D\}^{n}-\frac{1}{\Delta t^{2} \beta}\{D\}^{n-1}-\frac{1}{\Delta t \beta}\{\dot{D}\}^{n-1}-\frac{1}{2 \beta}(1-2 \beta)\{\ddot{D}\}^{n-1}
$$

and introducing this expression into the forward velocity of EQ. 41 yields

$$
\begin{aligned}
& \{\dot{D}\}^{n}=\{\dot{D}\}^{n-1}+\Delta t(1-\gamma)\{\ddot{D}\}^{n-1}+\frac{\gamma}{\Delta t \beta}\{D\}^{n}-\frac{\gamma}{\Delta t \beta}\{D\}^{n-1} \\
& -\frac{\gamma}{\beta}\{\dot{D}\}^{n-1}-\Delta t \gamma\left(\frac{1}{2 \beta}-1\right)\{\ddot{D}\}^{n-1}
\end{aligned}
$$

Substituting EQ. 42 and EQ. 43 into the system equation of motion, EQ. 39, yields

$$
\begin{aligned}
& {\left[\frac{1}{\Delta t^{2} \beta}[M]+\frac{\gamma}{\Delta t \beta}[C]+[K]\right]\{D\}^{n}=\{P\}^{n}+\left[\frac{1}{\Delta t^{2} \beta}[M]+\frac{\gamma}{\Delta t \beta}[C]\right]\{D\}^{n-1}} \\
& +\left[\frac{1}{\Delta t \beta}[M]-\left(1-\frac{\gamma}{B}\right)[C]\right]\{\dot{D}\}^{n-1}+\left[\left(\frac{1}{2 \beta}-1\right)[M]+\left(\frac{\Delta t \gamma}{2 \beta}-\Delta t\right)[C]\right]\{\ddot{D}\}^{n-1}
\end{aligned}
$$

EQ. 44 provides a recursion relationship for solving for the forward system displacement vector in terms of the forward load and the system displacement, velocity and acceleration vectors from the previous time steps. As a result of the linearity of the system, each time increment can be accomplished with a single step solution which requires an inversion to solve the system of equations indicate in EQ. 44. It is noted that the equation solution directly provides the total displacement of the system, and since the stiffness matrix of the linear system is constant (i.e. [K] is constant), the matrix on the left hand side can be formed, factored and stored in memory. Thus after the first time step, solution for subsequent time steps simply requires back-substitution with a new right hand side vector.

An alternate form of EQ. 44, in terms of displacement increment, can be obtained by recognizing $-[C]\{\dot{D}\}^{n-1}-[M]\{\ddot{D}\}^{n-1}=-\{P\}^{n-1}+[K]\{D\}^{n-1}$, then

$$
\begin{aligned}
& {\left[\frac{1}{\Delta t^{2} \beta}[M]+\frac{\gamma}{\Delta t \beta}[C]+[K]\right]\{\Delta D\}=\{P\}^{n}-\{P\}^{n-1}} \\
& +\left[\frac{1}{\Delta t \beta}[M]+\frac{\gamma}{\beta}[C]\right]\{\dot{D}\}^{n-1}+\left[\frac{1}{2 \beta}[M]+\left(\frac{\Delta t \gamma}{2 \beta}-\Delta t\right)[C]\right]\{\ddot{D}\}^{n-1}
\end{aligned}
$$

and 


$$
\{D\}^{n}=\{D\}^{n-1}+\{\Delta D\}
$$

and as before the constant matrix on the left hand side can be factored and stored once for the back substitutions of a new right hand side at each time step.

\subsubsection{Nonlinear time-history analysis}

In the nonlinear system model, the internal resisting forces cannot be written simply in terms of the stiffness matrix-displacement vector product. For a general nonlinear system, the governing equation of motion at time $\mathrm{n}$ can then be written,

$$
[M]\{\ddot{D}\}^{n}+[C]\{\dot{D}\}^{n}+\left\{Q\{D\}^{n}\right\}=\{P\}^{n}
$$

where $\left\{Q\{D\}^{n}\right\}$ is the vector of internal resisting forces at time $\mathrm{n}$.

The internal resisting forces at time $\mathrm{n}$ can be written in terms of the resisting forces at time $\mathrm{n}$ in a Taylor series expansion,

$$
\left\{Q\{D\}^{n}\right\}=\left\{Q\{D\}^{n-1}\right\}+\left[\frac{\partial}{\partial D} Q\{D\}^{n-1}\right]\{\Delta D\}+o(\Delta D)^{2}
$$

Where $\{\Delta D\}=\{D\}^{n}-\{D\}^{n-1}$, the incremental displacement vector between time $\mathrm{n}-1$ and time $\mathrm{n}$.

Neglecting higher order terms in EQ. 48 and substituting into the governing equation of motion of EQ. 47 yields,

$$
[M]\{\ddot{D}\}^{n}+[C]\{\dot{D}\}^{n}+\left[\frac{\partial}{\partial D} Q\{D\}^{n-1}\right]\{\Delta D\}=\left(\{P\}^{n}-\left\{Q\{D\}^{n-1}\right\}\right)
$$

Substituting the Newmark expressions of EQ. 42 and EQ. 43 into the equation of motion of EQ. 49 and performing algebraic simplification yields,

$$
\begin{aligned}
& {\left[\frac{1}{\Delta t^{2} \beta}[M]+\frac{\gamma}{\Delta t \beta}[C]+\left[\frac{\partial}{\partial D} Q\{D\}^{n-1}\right]\right]\{\Delta D\}=\{P\}^{n}-\left\{Q\{D\}^{n-1}\right\}} \\
& +\left[\frac{1}{\Delta t \beta}[M]-\left(1-\frac{\gamma}{\beta}\right)[C]\right]\{\dot{D}\}^{n-1}+\left[\left(\frac{1}{2 \beta}-1\right)[M]+\left(\frac{\Delta t \gamma}{2 \beta}-\Delta t\right)[C]\right]\{\ddot{D}\}^{n-1}
\end{aligned}
$$

EQ. 50 provides a recursion relationship for the incremental displacement between time n1 and time $n$. Denoting the displacement dependent rate of change of internal resisting forces with displacements by the instantaneous stiffness matrix $\left[K_{I}\{D\}\right], \mathrm{EQ} .50$ can be written, 


$$
\begin{aligned}
& {\left[\frac{1}{\Delta t^{2} \beta}[M]+\frac{\gamma}{\Delta t \beta}[C]+\left[K_{I}\{D\}^{n-1}\right]\right]\{\Delta D\}=\{P\}^{n}-\left\{Q\{D\}^{n-1}\right\}} \\
& +\left[\frac{1}{\Delta t \beta}[M]-\left(1-\frac{\gamma}{\beta}\right)[C]\right]\{\dot{D}\}^{n-1}+\left[\left(\frac{1}{2 \beta}-1\right)[M]+\left(\frac{\Delta t \gamma}{2 \beta}-\Delta t\right)[C]\right]\{\ddot{D}\}^{n-1}
\end{aligned}
$$

Recognizing that $-\left\{Q\{D\}^{n-1}\right\}-[C]\{\dot{D}\}^{n-1}-[M]\{\ddot{D}\}^{n-1}=-\{P\}^{n-1}$, EQ. 51 can be rewritten,

$$
\begin{aligned}
& {\left[\frac{1}{\Delta t^{2} \beta}[M]+\frac{\gamma}{\Delta t \beta}[C]+\left[K_{I}\{D\}^{n-1}\right]\right]\{\Delta D\}=\{P\}^{n}-\{P\}^{n-1}} \\
& +\left[\frac{1}{\Delta t \beta}[M]+\frac{\gamma}{\beta}[C]\right]\{\dot{D}\}^{n-1}+\left[\frac{1}{2 \beta}[M]+\left(\frac{\Delta t \gamma}{2 \beta}-\Delta t\right)[C]\right]\{\ddot{D}\}^{n-1}
\end{aligned}
$$

It is noted EQ. 52 for the nonlinear is similar to the incremental form for the linear system in EQ. 45, however unlike for the linear system the left hand side matrix must be reformed and factored at each time step owing to the changing, displacement dependent stiffness matrix.

\section{Equilibrium iterations to improve the displacement approximation}

Once a displacement increment across a time step is obtained from the Newmark time stepping procedure (i.e. EQ. 52 is solved for a displacement increment), Newton type iterations can be applied to achieve better equilibrium between the applied loads, inertial forces, resisting forces, and damping forces of the structure. Analogous to the static nonlinear problem, a system residual vector can be defined at the forward time $n$,

$$
\begin{aligned}
& \left\{R\left(\{D\}^{n},\{\dot{D}\}^{n},\{\ddot{D}\}^{n}\right)\right\}= \\
& \{P\}^{n}-\left\{Q\{D\}^{n}\right\}-[M]\{\ddot{D}\}^{n}-[C]\{\dot{D}\}^{n}
\end{aligned}
$$

The residual is explicitly a function of system displacements, velocities and accelerations at the forward time step n, however, the Newmark relationships give the velocity and acceleration at the forward time in terms of the forward displacement, and previous (backward) displacement, velocity and acceleration (see EQ. 42 and EQ. 43), i.e.

$$
\begin{aligned}
& \{\dot{D}\}^{n} \rightarrow f\left(\{D\}^{n},\{D\}^{n-1},\{\dot{D}\}^{n-1},\{\ddot{D}\}^{n-1}\right) \\
& \{\ddot{D}\}^{n} \rightarrow f\left(\{D\}^{n},\{D\}^{n-1},\{\dot{D}\}^{n-1},\{\ddot{D}\}^{n-1}\right)
\end{aligned}
$$

or, slightly rearranging EQ. 42 and EQ. 43 , 


$$
\begin{gathered}
\{\dot{D}\}^{n}=\frac{\gamma}{\Delta t \beta}\{D\}^{n}+\{\dot{D}\}^{n-1}+\Delta t(1-\gamma)\{\ddot{D}\}^{n-1}-\frac{\gamma}{\Delta t \beta}\{D\}^{n-1} \\
-\frac{\gamma}{\beta}\{\dot{D}\}^{n-1}-\Delta t \gamma\left(\frac{1}{2 \beta}-1\right)\{\ddot{D}\}^{n-1} \\
\{\ddot{D}\}^{n}=\frac{1}{\Delta t^{2} \beta}\{D\}^{n}-\frac{1}{\Delta t^{2} \beta}\{D\}^{n-1}-\frac{1}{\Delta t \beta}\{\dot{D}\}^{n-1}-\frac{1}{2 \beta}(1-2 \beta)\{\ddot{D}\}^{n-1}
\end{gathered}
$$

Substituting EQ. 56 and EQ. 57 into the residual expression in EQ. 53 yields,

$$
\begin{aligned}
& \left\{R\{D\}^{n}\right\}=\{P\}^{n}-\left\{Q\{D\}^{n}\right\}- \\
& {[M]\left\{\frac{1}{\Delta t^{2} \beta}\{D\}^{n}-\frac{1}{\Delta t^{2} \beta}\{D\}^{n-1}-\frac{1}{\Delta t \beta}\{\dot{D}\}^{n-1}-\frac{1}{2 \beta}(1-2 \beta)\{\ddot{D}\}^{n-1}\right\}-} \\
& {[C]\left\{\frac{\gamma}{\Delta t \beta}\{D\}^{n}+\{\dot{D}\}^{n-1}+\Delta t(1-\gamma)\{\ddot{D}\}^{n-1}-\frac{\gamma}{\Delta t \beta}\{D\}^{n-1}\right.} \\
& \left.-\frac{\gamma}{\beta}\{\dot{D}\}^{n-1}-\Delta t \gamma\left(\frac{1}{2 \beta}-1\right)\{\ddot{D}\}^{n-1}\right\}
\end{aligned}
$$

Invoking the Newmark formulas essentially allows the residual at the forward time step, $n$, to be written in terms of know quantities from the previous time step, n-1, and the unknown displacements at the forward time step (i.e. the velocity and acceleration at the forward time step are removed from the residual expression). Another interpretation is that the Newmark relationships have effectively provided a constraint between the inertial, damping and internal forces at the forward time step $n+1$.

Proceeding in a similar fashion to the nonlinear static problem, if $\left\{D^{\hbar k}\right\}^{n}$ corresponds to an equilibrium configuration at time " $n$ " and $\{D\}_{j}{ }^{n}$ represents the $\mathrm{jth}$ approximation to $\{D\}^{n}$, then a Taylor series expansion of the residual about $\{D\}_{j}{ }^{n}$ is given by

$$
\left\{R\left\{D^{\breve{2}}\right\}^{n}\right\}=\left\{R\{D\}_{j}^{n}\right\}+\left[\frac{\partial}{\partial D^{n}} R\left(\{D\}_{j}^{n}\right)\right]\{\Delta D\}_{j}+o(\Delta D)^{2}
$$

Substituting the residual expression of EQ. 58 into EQ. 59 and making use of the fact that $\left\{R\left\{D^{\hbar}\right\}^{n}\right\}=0$ yields, 
$\left\{Q\{D\}_{j}^{n}\right\}-\{P\}^{n}+$

(EQ 60)

$[M]\left\{\frac{1}{\Delta t^{2} \beta}\{D\}_{j}^{n}-\frac{1}{\Delta t^{2} \beta}\{D\}^{n-1}-\frac{1}{\Delta t \beta}\{\dot{D}\}^{n-1}-\frac{1}{2 \beta}(1-2 \beta)\{\ddot{D}\}^{n-1}\right\}+$

$[C]\left\{\frac{\gamma}{\Delta t \beta}\{D\}_{j}^{n}+\{\dot{D}\}^{n-1}+\Delta t(1-\gamma)\{\ddot{D}\}^{n-1}-\frac{\gamma}{\Delta t \beta}\{D\}^{n-1}\right.$

$\left.-\frac{\gamma}{\beta}\{\dot{D}\}^{n-1}-\Delta t \gamma\left(\frac{1}{2 \beta}-1\right)\{\ddot{D}\}^{n-1}\right\}=\left[-\frac{1}{\Delta t^{2} \beta}[M]+-\frac{\gamma}{\Delta t \beta}[C]-\frac{\partial}{\partial D} Q\{D\}_{j}^{n}\right]\{\Delta D\}_{j}$

or rearranging,

$$
\begin{aligned}
& {\left[\frac{1}{\Delta t^{2} \beta}[M]+\frac{\gamma}{\Delta t \beta}[C]+\frac{\partial}{\partial D} Q\{D\}_{j}^{n}\right]\{\Delta D\}_{j}=\{P\}^{n}-\left\{Q\{D\}_{j}^{n}\right\}-} \\
& {[M]\left\{\frac{1}{\Delta t^{2} \beta}\{D\}_{j}^{n}-\frac{1}{\Delta t^{2} \beta}\{D\}^{n-1}-\frac{1}{\Delta t \beta}\{\dot{D}\}^{n-1}-\frac{1}{2 \beta}(1-2 \beta)\{\ddot{D}\}^{n-1}\right\}-} \\
& {[C]\left\{\frac{\gamma}{\Delta t \beta}\{D\}_{j}^{n}+\{\dot{D}\}^{n-1}+\Delta t(1-\gamma)\{\ddot{D}\}^{n-1}-\frac{\gamma}{\Delta t \beta}\{D\}^{n-1}\right.} \\
& \left.-\frac{\gamma}{\beta}\{\dot{D}\}^{n-1}-\Delta t \gamma\left(\frac{1}{2 \beta}-1\right)\{\ddot{D}\}^{n-1}\right\}
\end{aligned}
$$

and EQ. 61 can simply be written

$$
\begin{aligned}
& {\left[\frac{1}{\Delta t^{2} \beta}[M]+\frac{\gamma}{\Delta t \beta}[C]+\left[K_{I}\{D\}_{j}^{n}\right]\right]\{\Delta D\}_{j}=\{P\}^{n}} \\
& -[M]\{\ddot{D}\}_{j}^{n}-[C]\{\dot{D}\}_{j}^{n}-\left\{Q\{D\}_{j}^{n}\right\}
\end{aligned}
$$

where the right hand side is simply the residual of the equations of motion. EQ. 62 can be utilized to iteratively improve the displacement approximation $\{D\}_{j}{ }^{n}$. The NewmarkBeta integration with equilibrium iterations then consists of determining the initial displacement increment $\{\Delta D\}$ across the time step with EQ. 52, and updating the displacement velocity and acceleration vectors to obtain the first approximation of these quantities at the forward time step, i.e.

$$
\{D\}_{1}^{n}=\{D\}^{n-1}+\{\Delta D\}
$$




$$
\begin{gathered}
\{\dot{D}\}_{1}^{n}=\frac{\gamma}{\Delta t \beta}\{D\}_{0}^{n}+\{\dot{D}\}^{n-1}+\Delta t(1-\gamma)\{\ddot{D}\}^{n-1}-\frac{\gamma}{\Delta t \beta}\{D\}^{n-1} \\
-\frac{\gamma}{\beta}\{\dot{D}\}^{n-1}-\Delta t \gamma\left(\frac{1}{2 \beta}-1\right)\{\ddot{D}\}^{n-1} \\
\{\ddot{D}\}_{1}^{n}=\frac{1}{\Delta t^{2} \beta}\{D\}_{0}^{n}-\frac{1}{\Delta t^{2} \beta}\{D\}^{n-1}-\frac{1}{\Delta t \beta}\{\dot{D}\}^{n-1}-\frac{1}{2 \beta}(1-2 \beta)\{\ddot{D}\}^{n-1}
\end{gathered}
$$

The vectors $\{D\}_{1}^{n},\{\dot{D}\}_{1}^{n}$ and $\{\ddot{D}\}_{1}^{n}$ then become the first estimates to enter the residual calculation in EQ. 62.

A summary of the solution algorithms is given in Figure 17. 
Static Analyses

- Linear systems...

- Nonlinear systems...

$$
[K]\{D\}=\{P\}
$$

- - - - . - .

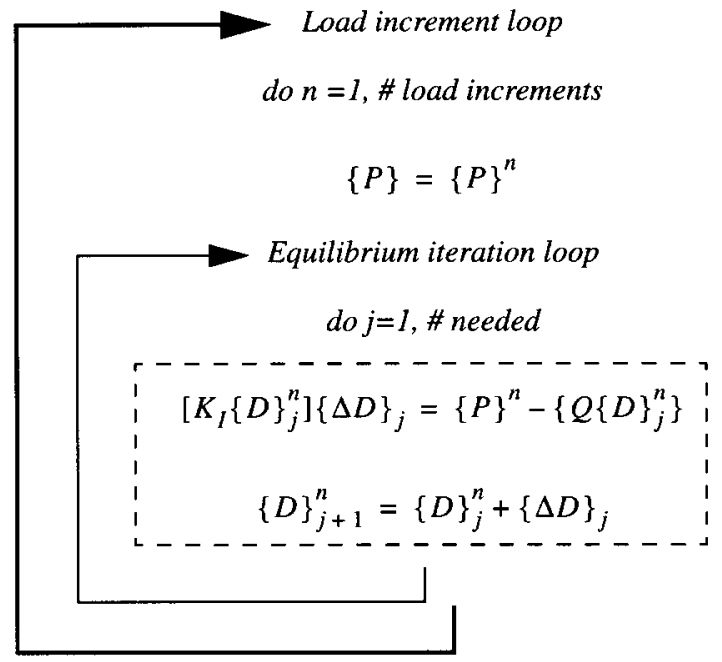

\section{Dynamic Analyses}

- Linear systems...

Time step loop

do $n=1$, ntimesteps

$$
\{P\}=\{P\}^{n}
$$

$$
\begin{aligned}
& {\left[\frac{1}{\Delta t^{2} \beta}[M]+\frac{\gamma}{\Delta t \beta}[C]+[K]\right]\{\Delta D\}=\{P\}^{n}-\{P\}^{n-1}} \\
& +\left[\frac{1}{\Delta t \beta}[M]+\frac{\gamma}{\beta}[C]\right]\{\dot{D}\}^{n-1}+\left[\frac{1}{2 \beta}[M]+\left(\frac{\Delta t \gamma}{2 \beta}-\Delta t\right)[C]\right]\{\ddot{D}\}^{n-1} \\
& \{D\}^{n}=\{D\}^{n-1}+\{\Delta D\} \\
& \{\dot{D}\}^{n}=\frac{\gamma}{\Delta t \beta}\{D\}^{n}+\{\dot{D}\}^{n-1}+\Delta t(1-\gamma)\{\ddot{D}\}^{n-1}-\frac{\gamma}{\Delta t \beta}\{D\}^{n-1} \\
& -\frac{\gamma}{\beta}\{\dot{D}\}^{n-1}-\Delta t \gamma\left(\frac{1}{2 \beta}-1\right)\{\ddot{D}\}^{n-1} \\
& \{\ddot{D}\}^{n}=\frac{1}{\Delta t^{2} \beta}\{D\}^{n}-\frac{1}{\Delta t^{2} \beta}\{D\}^{n-1}-\frac{1}{\Delta t \beta}\{\dot{D}\}^{n-1}-\frac{1}{2 \beta}(1-2 \beta)\{\ddot{D}\}^{n-1} \\
& \text { ᄂ - - - - - - - - - - - - - - - - - . - . }
\end{aligned}
$$


Dynamic Analyses (cont.)

- Nonlinear systems...

Time stepping loop

do $n=1$, \# time steps

$$
\{P\}=\{P\}^{n}
$$

$$
\begin{gathered}
{\left[\frac{1}{\Delta t^{2} \beta}[M]+\frac{\gamma}{\Delta t \beta}[C]+\left[K_{I}\{D\}^{n-1}\right]\right]\{\Delta D\}=\{P\}^{n}-\{P\}^{n-1}} \\
+\left[\frac{1}{\Delta t \beta}[M]+\frac{\gamma}{\beta}[C]\right]\{\dot{D}\}^{n-1}+\left[\frac{1}{2 \beta}[M]+\left(\frac{\Delta t \gamma}{2 \beta}-\Delta t\right)[C]\right]\{\ddot{D}\}^{n-1} \\
\{D\}_{1}^{n}=\{D\}^{n-1}+\{\Delta D\}
\end{gathered}
$$$$
\{\dot{D}\}_{1}^{n}=\frac{\gamma}{\Delta t \beta}\{D\}_{1}^{n}+\{\dot{D}\}^{n-1}+\Delta t(1-\gamma)\{\ddot{D}\}^{n-1}-\frac{\gamma}{\Delta t \beta}\{D\}^{n-1}
$$$$
-\frac{\gamma}{\beta}\{\dot{D}\}^{n-1}-\Delta t \gamma\left(\frac{1}{2 \beta}-1\right)\{\ddot{D}\}^{n-1}
$$

$\{\ddot{D}\}_{1}^{n}=\frac{1}{\Delta t^{2} \beta}\{D\}_{1}^{n}-\frac{1}{\Delta t^{2} \beta}\{D\}^{n-1}-\frac{1}{\Delta t \beta}\{\dot{D}\}^{n-1}-\frac{1}{2 \beta}(1-2 \beta)\{\ddot{D}\}^{n-1}$

Equilibrium iteration loop

\section{do $j=1$, \# needed}

$$
\begin{gathered}
{\left[\frac{1}{\Delta t^{2} \beta}[M]+\frac{\gamma}{\Delta t \beta}[C]+\left[K_{I}\{D\}_{j}^{n}\right]\right]\{\Delta D\}_{j}=\{P\}^{n}} \\
-[M]\{\ddot{D}\}_{j}^{n}-[C]\{\dot{D}\}_{j}^{n}-\left\{Q\{D\}_{j}^{n}\right\} \\
\{D\}_{j+1}^{n}=\{D\}_{j}^{n}+\{\Delta D\}_{j} \\
\{\dot{D}\}_{j+1}^{n}=\frac{\gamma}{\Delta t \beta}\{D\}_{j}^{n}+\{\dot{D}\}^{n-1}+\Delta t(1-\gamma)\{\ddot{D}\}^{n-1}-\frac{\gamma}{\Delta t \beta}\{D\}^{n-1} \\
-\frac{\gamma}{\beta}\{\dot{D}\}^{n-1}-\Delta t \gamma\left(\frac{1}{2 \beta}-1\right)\{\ddot{D}\}^{n-1}
\end{gathered}
$$$$
\{\ddot{D}\}_{j+1}^{n}=\frac{1}{\Delta t^{2} \beta}\{D\}_{j+1}^{n}-\frac{1}{\Delta t^{2} \beta}\{D\}^{n-1}-\frac{1}{\Delta t \beta}\{\dot{D}\}^{n-1}-\frac{1}{2 \beta}(1-2 \beta)\{\ddot{D}\}^{n-1}
$$

FIGURE 17. Summary of solution algorithms for static and dynamic, linear and nonlinear problems. 


\subsection{Local-global coordinate transformation}

In order to develop the system coordinate transformations for a one dimensional element with a convected local coordinate system, the direction cosines and transformation matrices must be derived. The transformation matrices, the components of which are the direction cosines of the axes, define the transformation of vector quantities between coordinate systems. Consider the one dimensional element shown in Figure 18.
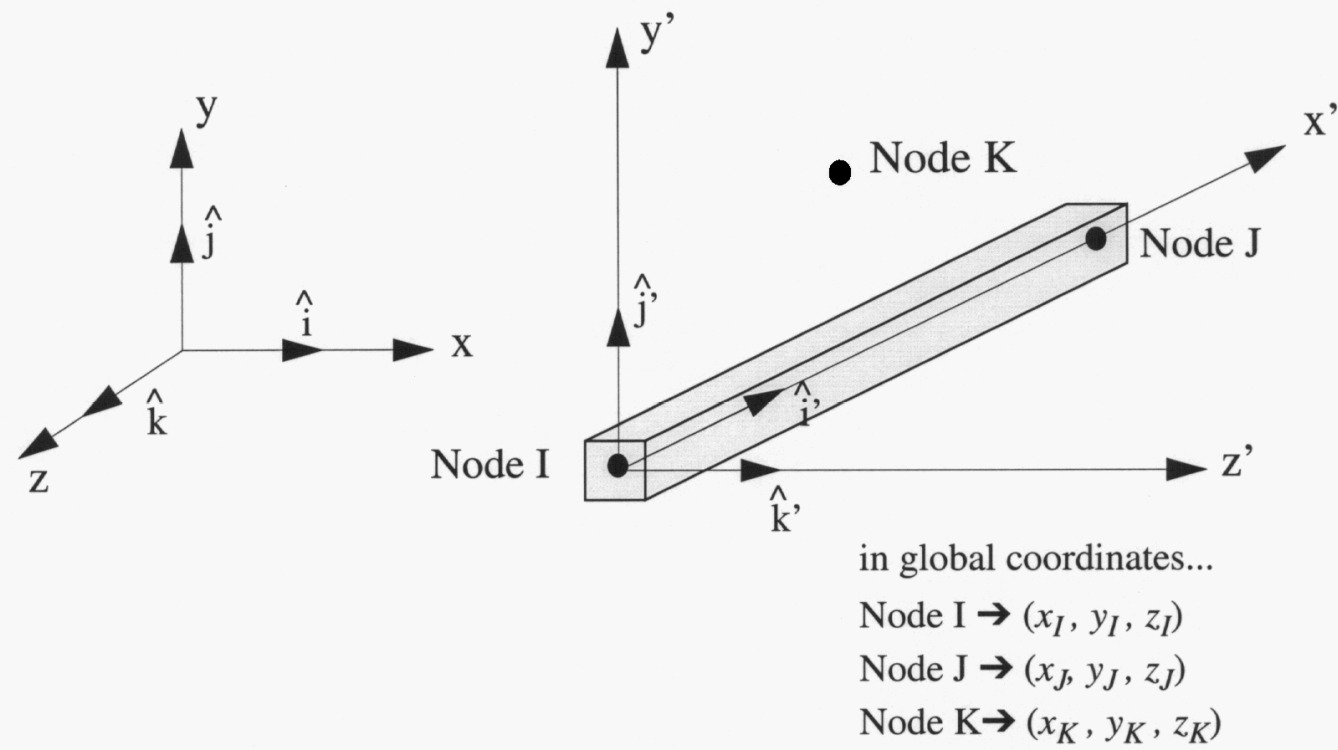

FIGURE 18. Global and local coordinate

Axis $\mathrm{x}^{\prime}$ is defined in terms of the unit vector $\hat{i}^{\prime}$,

$$
\hat{i}^{\prime}=l_{x^{\prime}} \hat{i}+m_{x^{\prime}} \hat{j}+n_{x^{\prime}} \hat{k}
$$

where,

$$
\begin{aligned}
& l_{x^{\prime}}=\frac{x_{J}-x_{I}}{\sqrt{\left(x_{J}-x_{I}\right)^{2}+\left(y_{J}-y_{I}\right)^{2}+\left(z_{J}-z_{I}\right)^{2}}} \\
& m_{x^{\prime}}=\frac{y_{J}-y_{I}}{\sqrt{\left(x_{J}-x_{I}\right)^{2}+\left(y_{J}-y_{I}\right)^{2}+\left(z_{J}-z_{I}\right)^{2}}} \\
& n_{x^{\prime}}=\frac{z_{J}-z_{I}}{\sqrt{\left(x_{J}-x_{I}\right)^{2}+\left(y_{J}-y_{I}\right)^{2}+\left(z_{J}-z_{I}\right)^{2}}}
\end{aligned}
$$

Based on vectors from $\mathrm{I}$ to $\mathrm{J}$ and from $\mathrm{I}$ to $\mathrm{K}$, the unit vector in the $\mathrm{z}$ ' direction can be obtained,

$$
\overrightarrow{I K}=\left(x_{K}-x_{I}\right) \hat{i}+\left(y_{K}-y_{I}\right) \hat{j}+\left(z_{K}-z_{I}\right) \hat{k}
$$




$$
\overrightarrow{I J}=\left(x_{J}-x_{I}\right) \hat{i}+\left(y_{J}-y_{I}\right) \hat{j}+\left(z_{J}-z_{I}\right) \hat{k}
$$

the cross product of IK and IJ is given by,

$$
\begin{aligned}
\overrightarrow{I J} \otimes \overrightarrow{I K}= & \left(x_{J}-x_{I}\right) \hat{i}+\left(y_{J}-y_{I}\right) \hat{j}+\left(z_{J}-z_{I}\right) \hat{k} \otimes \\
& \left(x_{K}-x_{I}\right) \hat{i}+\left(y_{K}-y_{I}\right) \hat{j}+\left(z_{K}-z_{I}\right) \hat{k}
\end{aligned}
$$

thus,

$$
\begin{gathered}
\overrightarrow{I J} \otimes \overrightarrow{I K}=\left[\left(y_{J}-y_{I}\right)\left(z_{K}-z_{I}\right)-\left(z_{J}-z_{I}\right)\left(y_{K}-y_{I}\right)\right] \hat{i}+ \\
{\left[-\left(x_{J}-x_{I}\right)\left(z_{K}-z_{I}\right)+\left(z_{J}-z_{I}\right)\left(x_{K}-x_{I}\right)\right] \hat{j}+} \\
{\left[\left(x_{J}-x_{I}\right)\left(y_{K}-y_{I}\right)-\left(y_{J}-y_{I}\right)\left(x_{K}-x_{I}\right)\right] \hat{k}}
\end{gathered}
$$

Now, unitize the product to get the unit vector in the z' direction, if

$$
\overrightarrow{I J} \otimes \overrightarrow{I K}=C_{1} \hat{i}+C_{2} \hat{j}+C_{3} \hat{k}
$$

then

$$
\hat{k}^{\prime}=\frac{C_{1}}{\sqrt{C_{1}^{2}+C_{2}^{2}+C_{3}^{2}}} \hat{i}+\frac{C_{2}}{\sqrt{C_{1}^{2}+C_{2}^{2}+C_{3}^{2}}} \hat{j}+\frac{C_{3}}{\sqrt{C_{1}^{2}+C_{2}^{2}+C_{3}^{2}}} \hat{k}
$$

or

$$
\hat{k}^{\prime}=l_{z^{\prime}} \hat{i}+m_{z^{\prime}} \hat{j}+n_{z^{\prime}} \hat{k}
$$

where

$$
\begin{aligned}
& l_{z^{\prime}}=\frac{C_{1}}{\sqrt{C_{1}^{2}+C_{2}^{2}+C_{3}^{2}}} \\
& m_{z^{\prime}}=\frac{C_{2}}{\sqrt{C_{1}^{2}+C_{2}^{2}+C_{3}^{2}}} \\
& n_{z^{\prime}}=\frac{C_{3}}{\sqrt{C_{1}^{2}+C_{2}^{2}+C_{3}^{2}}}
\end{aligned}
$$

To get the unit vector in the y' direction, construct the cross product $\hat{k}^{\prime} \otimes \hat{i}^{\prime}$,

$$
\begin{aligned}
& \hat{j}^{\prime}=\hat{k}^{\prime} \otimes \hat{i}^{\prime}=l_{z^{\prime}} \hat{i}+m_{z^{\prime}} \hat{j}+n_{z^{\prime}} \hat{k} \otimes l_{x^{\prime}} \hat{i}+m_{x^{\prime}} \hat{j}+n_{x^{\prime}} \hat{k}= \\
& \left(m_{z^{\prime}} n_{x^{\prime}}-n_{z^{\prime}} m_{x^{\prime}}\right) \hat{i}+\left(-l_{z^{\prime}} n_{x^{\prime}}+n_{z^{\prime}} l_{x^{\prime}}\right) \hat{j}+\left(l_{z^{\prime}} m_{x^{\prime}}-m_{z^{\prime}} l_{x^{\prime}}\right) \hat{k}
\end{aligned}
$$

or 


$$
\hat{j}^{\prime}=l_{y^{\prime}} \hat{i}+m_{y^{\prime}} \hat{j}+n_{y^{\prime}} \hat{k}
$$

where

$$
\begin{aligned}
& l_{y^{\prime}}=\left(m_{z^{\prime}} n_{x^{\prime}}-n_{z^{\prime}} m_{x^{\prime}}\right) \\
& m_{y^{\prime}}=\left(-l_{z^{\prime}} n_{x^{\prime}}+n_{z^{\prime}} l_{x^{\prime}}\right) \\
& n_{y^{\prime}}=\left(l_{z^{\prime}} m_{x^{\prime}}-m_{z^{\prime}} l_{x^{\prime}}\right)
\end{aligned}
$$

A vector quantity $\vec{D}$ can now be described in either coordinate system shown in Figure 19,
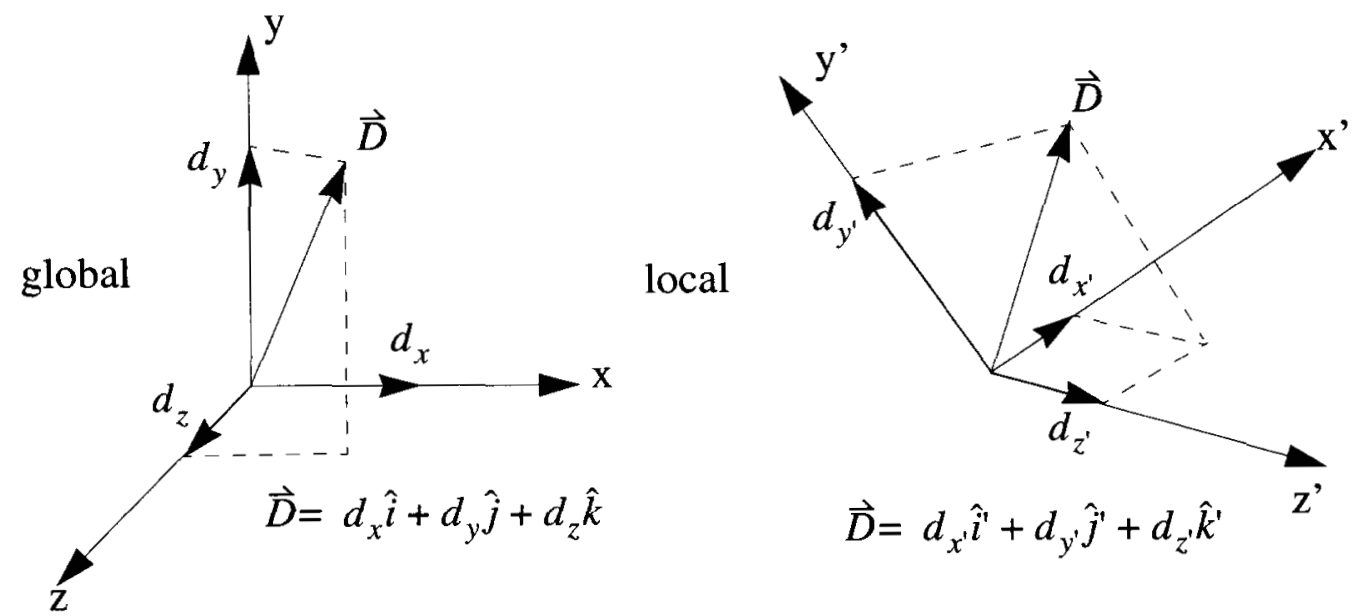

FIGURE 19. Vector quantity in global and local coordinates

given $\vec{D}=d_{x^{\prime}} \hat{i}^{\prime}+d_{y^{\prime}} \hat{j}^{\prime}+d_{z^{\prime}} \hat{k}^{\prime}$, then

$$
d_{x}=\vec{D} \bullet \hat{i}
$$

thus,

$$
\begin{gathered}
d_{x}=\left(d_{x^{\prime}} \hat{i}^{\prime}+d_{y^{\prime}} \hat{j}^{\prime}+d_{z^{\prime}} \hat{k}^{\prime}\right) \bullet \hat{i} \\
\therefore d_{x}=d_{x^{\prime}}\left(l_{x^{\prime}} \hat{i}+m_{x^{\prime}} \hat{j}+n_{x^{\prime}} \hat{k}\right) \bullet \hat{i} \\
+d_{y^{\prime}}\left(l_{y^{\prime}} \hat{i}+m_{y^{\prime}} \hat{j}+n_{y^{\prime}} \hat{k}\right) \bullet \hat{i} \\
+d_{z^{\prime}}\left(l_{z^{\prime}} \hat{i}+m_{z^{\prime}} \hat{j}+n_{z^{\prime}} \hat{k}\right) \bullet j^{\prime} \\
\therefore d_{x}=d_{x^{\prime}} l_{x^{\prime}}+d_{y^{\prime}} l_{y^{\prime}}+d_{z^{\prime}} l_{z^{\prime}}
\end{gathered}
$$


similarly,

$$
\begin{gathered}
d_{y}=d_{x^{\prime}} m_{x^{\prime}}+d_{y^{\prime}} m_{y^{\prime}}+d_{z^{\prime}} m_{z^{\prime}} \\
d_{z}=d_{x^{\prime}} n_{x^{\prime}}+d_{y^{\prime}} n_{y^{\prime}}+d_{z^{\prime}} n_{z^{\prime}}
\end{gathered}
$$

In matrix form these relationships become

$$
\left[\begin{array}{l}
d_{x} \\
d_{y} \\
d_{z}
\end{array}\right]=\left[\begin{array}{ccc}
l_{x^{\prime}} & l_{y^{\prime}} & l_{z^{\prime}} \\
m_{x^{\prime}} & m_{y^{\prime}} & m_{z^{\prime}} \\
n_{x^{\prime}} & n_{y^{\prime}} & n_{z^{\prime}}
\end{array}\right]\left[\begin{array}{l}
d_{x^{\prime}} \\
d_{y^{\prime}} \\
d_{z^{\prime}}
\end{array}\right]
$$

or

$$
\{d\}=[T]^{T}\left\{d^{\prime}\right\}
$$

The inverse transformation is given by,

$$
\begin{aligned}
d_{x^{\prime}}=\vec{D} \bullet \hat{i}^{\prime}= & \left(d_{x} \hat{i}+d_{y} \hat{j}+d_{z} \hat{k}\right) \bullet\left(l_{x^{\prime}} \hat{i}+m_{x^{\prime}} \hat{j}+n_{x^{\prime}} \hat{k}\right) \\
& =d_{x} l_{x^{\prime}}+d_{y} m_{x^{\prime}}+d_{z} n_{x^{\prime}}
\end{aligned}
$$

similarly,

$$
\begin{aligned}
& d_{y^{\prime}}=d_{x} l_{y^{\prime}}+d_{y} m_{y^{\prime}}+d_{z} n_{y^{\prime}} \\
& d_{z^{\prime}}=d_{x} l_{z^{\prime}}+d_{y} m_{z^{\prime}}+d_{z} n_{z^{\prime}}
\end{aligned}
$$

or in matrix form

$$
\left[\begin{array}{l}
d_{x^{\prime}} \\
d_{y^{\prime}} \\
d_{z^{\prime}}
\end{array}\right]=\left[\begin{array}{lll}
l_{x^{\prime}} & m_{x^{\prime}} & n_{x^{\prime}} \\
l_{y^{\prime}} & m_{y^{\prime}} & n_{y^{\prime}} \\
l_{z^{\prime}} & m_{z^{\prime}} & n_{z^{\prime}}
\end{array}\right]\left[\begin{array}{l}
d_{x} \\
d_{y} \\
d_{z}
\end{array}\right]
$$

or

$$
\left\{d^{\prime}\right\}=[T]\{d\}
$$

EQ. 91 and EQ. 97 provide the transformation between global and local coordinates for any vector quantity. For example, these relationships provide the transformation between local and global coordinates for the end displacement and force vectors for a one dimensional truss element as shown in Figure 20. 


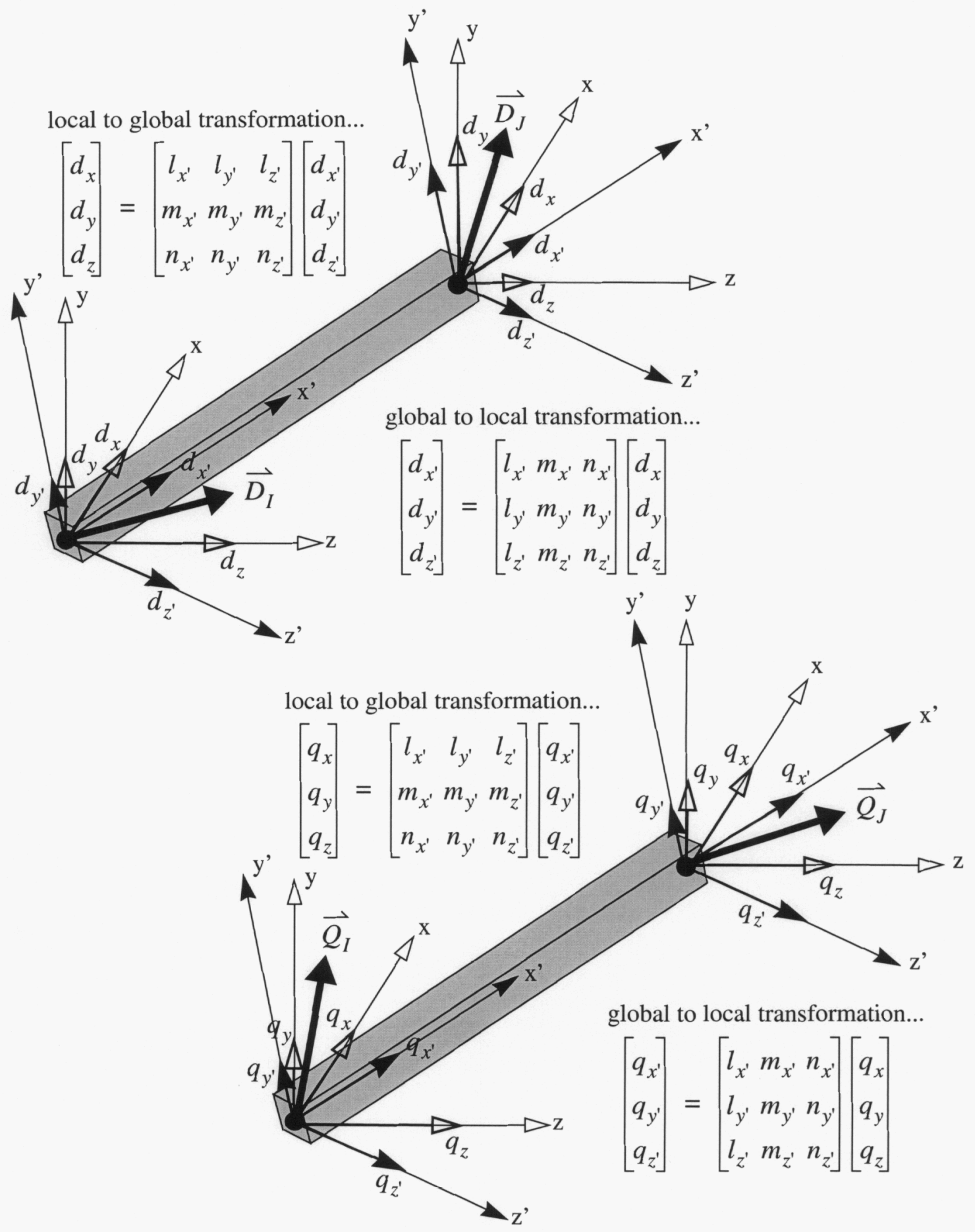

FIGURE 20. Application of coordinate transformation relationships to a one dimensional member 


\subsection{Nonlinear beam element}

\subsubsection{Engineering theory of beam bending}

Initially, a long slender beam ${ }^{1}$ will be considered in which the two transverse dimensions are much smaller than the longitudinal dimension as shown in Figure 21. The beam is subjected to both transverse and longitudinal forces. Based on physical observations of flexure of slender beams, the following fundamental observations about slender beam bending can be made;

- Sections plane and perpendicular to longitudinal fibers of the beam before deformation remain plane and perpendicular to the longitudinal fibers of the beam after deformation

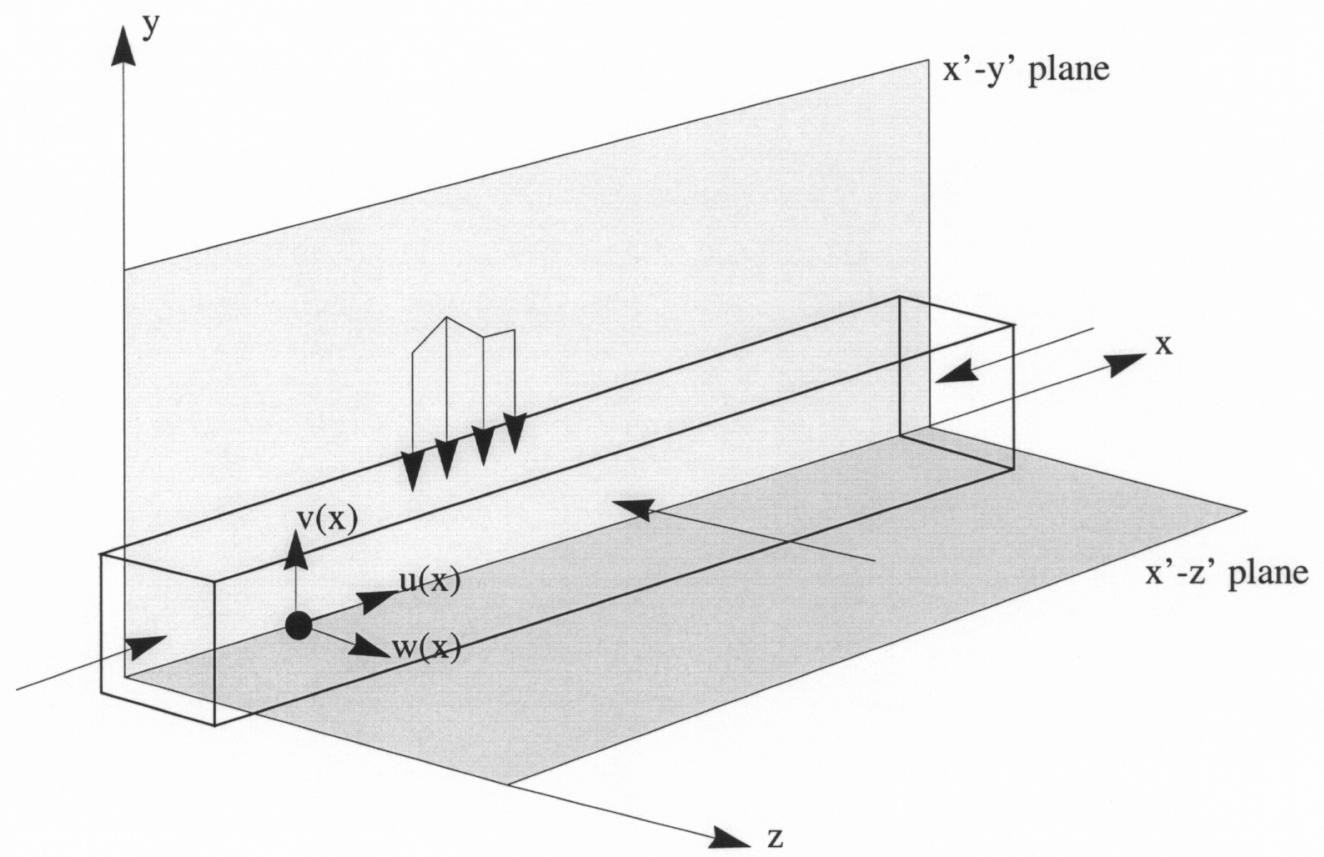

FIGURE 21. Slender beam subjected to transverse and longitudinal loads and the beam reference axes

- The transverse strains in the beam cross section are negligibly small and the cross section dimensions can be assumed constant (i.e. $\varepsilon_{y} \approx \varepsilon_{z} \approx 0$ )

The kinematics which result from these observations allows the development of a simple engineering theory of beam bending. Exploitation of these observations allows reduction of slender beam bending from a problem of three dimensional solid mechanics to a simple one dimensional engineering theory of beam bending.

1. For the discussion here, slender refers to beams for which the length is at least ten times the largest transverse dimension. 


\subsubsection{Beam theory kinematics}

To develop the simple bending theory, a set of reference axes will be defined as shown in Figure 22. To begin with, the location of the reference axes is arbitrary, the axes simply originate somewhere in the beam cross section at one end of the beam, and they are aligned with the primary beam directions as shown in Figure 22.

The displacement of the beam is defined in terms of the displacements of the reference axis, as given by the three displacement components $\mathrm{u}, \mathrm{v}$ and $\mathrm{w}$ as shown in Figure 22. The displacement of any point located off of the reference axes can be described in terms of the reference axis displacements. Consider any cross section of the beam undergoing deformation as shown in Figure 22. Taking into account the fact that transverse strains are neg-

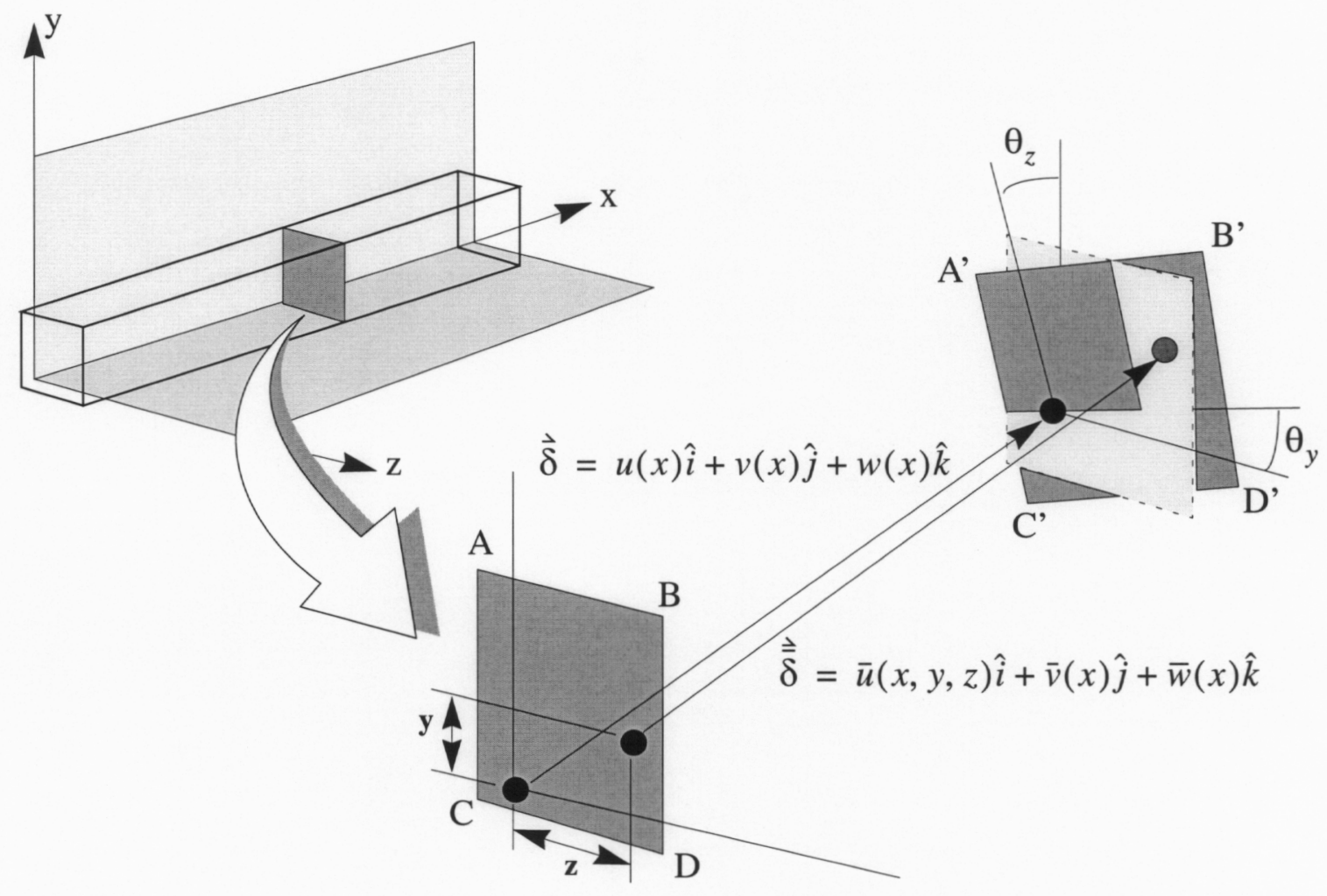

FIGURE 22. Displacements of points on and off the reference axes

ligibly small (the second fundamental observation of slender beam behavior) the off axis displacements in the $\mathrm{y}$ and $\mathrm{z}$ directions are taken equal to the reference axis displacements and thus only the longitudinal displacement is a function of all three spatial coordinates $\mathrm{x}$, $\mathrm{y}$, and $\mathrm{z}$. Thus,

$$
\begin{gathered}
\bar{u}=f(x, y, z) \\
u=f(x) \text { only } \\
\bar{v}=v=f(x) \text { only }
\end{gathered}
$$




$$
\bar{w}=w=f(x) \text { only }
$$

The displacement of any point on the reference axis is denoted by

$$
\vec{\delta}=u(x) \hat{i}+v(x) \hat{j}+w(x) \hat{k}
$$

and the displacement of any point off the reference axis is denoted by

$$
\overrightarrow{\bar{\delta}}=\bar{u}(x, y, z) \hat{i}+\bar{v}(x) \hat{j}+\bar{w}(x) \hat{k}
$$

Where the overbars are used to indicate a point off of the reference axis.

The longitudinal strain in fibers located either off or on the longitudinal axis can be written in terms of the displacement quantities. Consider two fiber segments, each with initial length $d x$, undergoing deformation as shown in Figure 23. The displacement of an axis fiber at the initial end is given by

$$
\vec{\delta}(x)=u(x) \hat{i}+v(x) \hat{j}+w(x) \hat{k}
$$

and the displacement at the far end of the fiber is given by

$$
\begin{aligned}
\vec{\delta}(x+d x)= & \left(u+\frac{\partial u}{\partial x} d x+\frac{1}{2}\left(\frac{\partial^{2} u}{\partial x^{2}}\right) d x^{2}+\ldots\right) \hat{i}+ \\
& \left(v+\frac{\partial v}{\partial x} d x+\frac{1}{2}\left(\frac{\partial^{2} v}{\partial x^{2}}\right) d x^{2}+\ldots\right) \hat{j}+ \\
& \left(w+\frac{\partial w}{\partial x} d x+\frac{1}{2}\left(\frac{\partial^{2} w}{\partial x^{2}}\right) d x^{2}+\ldots\right) \hat{j}
\end{aligned}
$$

Where the displacements in EQ. 105 have been expanded about the initial end of the fiber in a Taylor series. From vector addition, the vector defining the new fiber location can be found,

or

$$
\vec{\delta}(x)+\vec{\Psi}(x)=d x \hat{i}+\vec{\delta}(x+d x)
$$

$$
\vec{\Psi}(x)=d x \hat{i}+\vec{\delta}(x+d x)-\vec{\delta}(x)
$$

Neglecting higher order terms, the new length of the fiber is approximately equal to,

$$
l_{n e w}=|\vec{\Psi}(x)|=\sqrt{\left(d x+u+\frac{d u}{d x} d x-u\right)^{2}+\left(v+\frac{d v}{d x} d x-v\right)^{2}+\left(w+\frac{d w}{d x} d x-w\right)^{2}}
$$

or

$$
l_{n e w}=\sqrt{\left(d x+\frac{d u}{d x} d x\right)^{2}+\left(\frac{d v}{d x} d x\right)^{2}+\left(\frac{d w}{d x} d x\right)^{2}}-d x
$$

and the fiber strain is given by, 


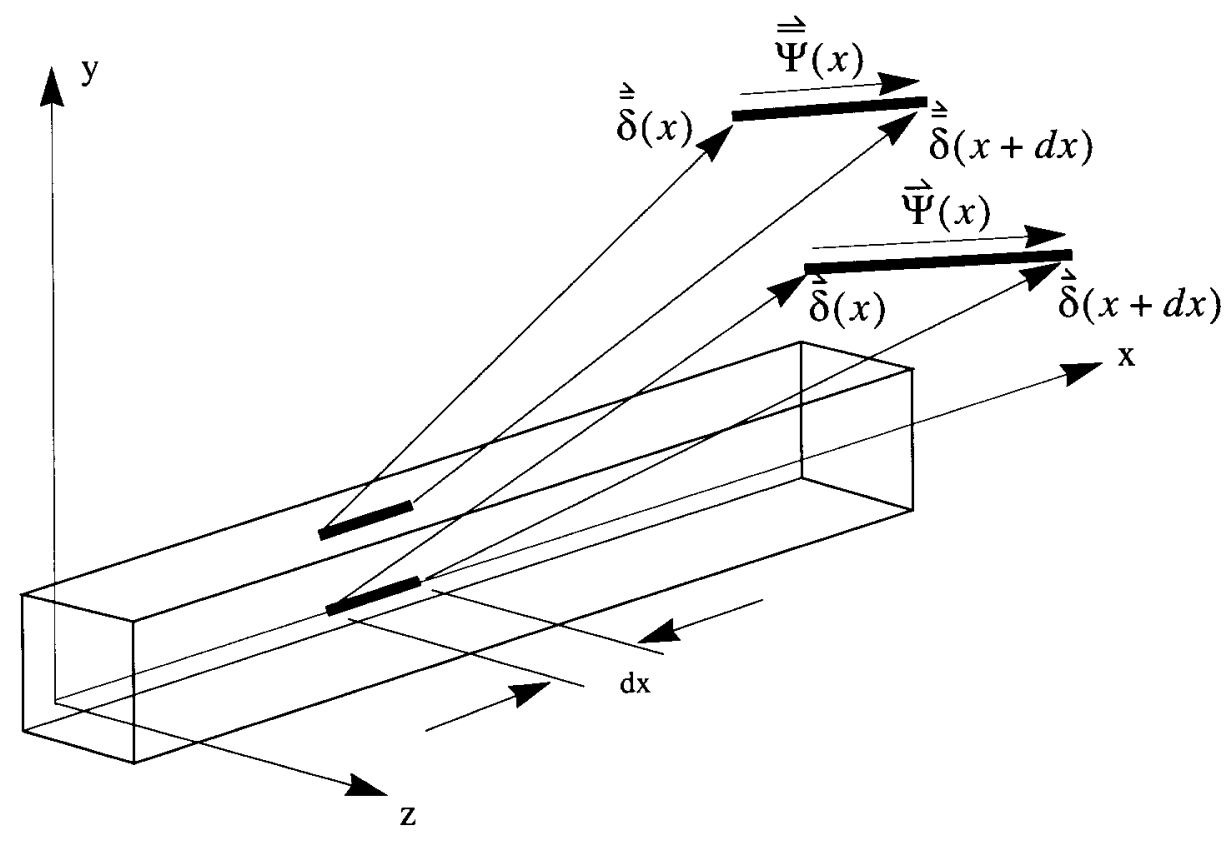

FIGURE 23. Deformation of fibers located on and off of the reference axis

$$
\varepsilon_{x}=\sqrt{\left(1+\frac{d u}{d x}\right)^{2}+\left(\frac{d v}{d x}\right)^{2}+\left(\frac{d w}{d x}\right)^{2}}-1
$$

or

$$
\varepsilon_{x}=\sqrt{\left(1+2 \frac{d u}{d x}+\left(\frac{d u}{d x}\right)^{2}\right)+\left(\frac{d v}{d x}\right)^{2}+\left(\frac{d w}{d x}\right)^{2}}-1
$$

Neglecting the higher order term $\left(\frac{d u}{d x}\right)^{2}$ but keeping the higher order terms $\left(\frac{d v}{d x}\right)^{2}$ and $\left(\frac{d w}{d x}\right)^{2}$ (this term will ultimately lead to inclusion of the geometric stiffness), the strain of the fiber is given by

$$
\varepsilon_{x}=\sqrt{\left(1+\left(2 \frac{d u}{d x}+\left(\frac{d v}{d x}\right)^{2}+\left(\frac{d w}{d x}\right)^{2}\right)\right)}-1
$$

Expanding EQ. 112 using the binomial expansion, ${ }^{1}$

$$
\varepsilon_{x}=1+\frac{1}{2}\left(2 \frac{d u}{d x}+\left(\frac{d v}{d x}\right)^{2}+\left(\frac{d w}{d x}\right)^{2}\right)+(\text { higherorderterms })-1
$$

or

1. $(1 \pm x)^{\frac{1}{2}}=1 \pm \frac{1}{2} x-\frac{1}{8} x^{2} \pm \ldots$ 


$$
\varepsilon_{x}=\frac{d u}{d x}+\frac{1}{2}\left(\frac{d v}{d x}\right)^{2}+\frac{1}{2}\left(\frac{d w}{d x}\right)^{2}
$$

Similarly, the new length of the off-axis fiber is given by,

$$
l_{n e w}=\sqrt{\left(d x+\bar{u}+\frac{\partial \bar{u}}{\partial x} d x-\bar{u}\right)^{2}+\left(\bar{v}+\frac{d}{d x} \bar{v} d x-\bar{v}\right)^{2}+\left(\bar{w}+\frac{d}{d x} \bar{w} d x-\bar{w}\right)^{2}}
$$

Which after similar expansion finally yields,

$$
\bar{\varepsilon}_{x}=\frac{\partial \bar{u}}{\partial x}+\frac{1}{2}\left(\frac{d v}{d x}\right)^{2}+\frac{1}{2}\left(\frac{d w}{d x}\right)^{2}
$$

Where use has been made of the fact that $\bar{v}=v$ and $\bar{w}=w$.

The off axis displacement can be written in terms of the reference axis displacement,

$$
\bar{u}=u-\theta_{z} y+\theta_{y} z
$$

For small deformations and small displacements, the rotations can be approximated by

$$
\begin{gathered}
\theta_{y} \approx \frac{d w}{d x} \\
\theta_{z} \approx \frac{d v}{d x}
\end{gathered}
$$

EQ. 118 and EQ. 119, EQ. 117 can be written

$$
\bar{u}=u-\frac{d v}{d x} y-\frac{d w}{d x} z
$$

Based on EQ. 120, EQ. 100 and EQ. 101, the displacements at points located off of the reference axis have been completely stated in terms of reference axis displacements. This is a direct consequence of the law of plane sections and is the key to reduction from a three dimensional continuum theory to a one dimensional engineering theory. EQ. 116 then becomes,

$$
\bar{\varepsilon}_{x}=\frac{d u}{d x}+\frac{1}{2}\left(\frac{d v}{d x}\right)^{2}+\frac{1}{2}\left(\frac{d w}{d x}\right)^{2}-\left(\frac{d^{2} v}{d x^{2}}\right) y-\left(\frac{d^{2} w}{d x^{2}}\right) z
$$

which can be written,

$$
\bar{\varepsilon}_{x}=\varepsilon_{x}-\kappa_{y} y-\kappa_{z} z
$$

\subsubsection{Beam theory stress resultants}

The beam theory stress resultants are shown in Figure 24. The stress resultants are given by

$$
\begin{gathered}
F_{x}=\int_{A} \bar{\sigma}_{x} d A \\
M_{y}=-\int_{A} \bar{\sigma}_{x} y d A
\end{gathered}
$$




$$
\begin{gathered}
M_{z}=-\int_{A} \bar{\sigma}_{x} z d A \\
T_{x}=\int_{A}\left(\bar{\tau}_{x z} y-\bar{\tau}_{x y} z\right) d A
\end{gathered}
$$

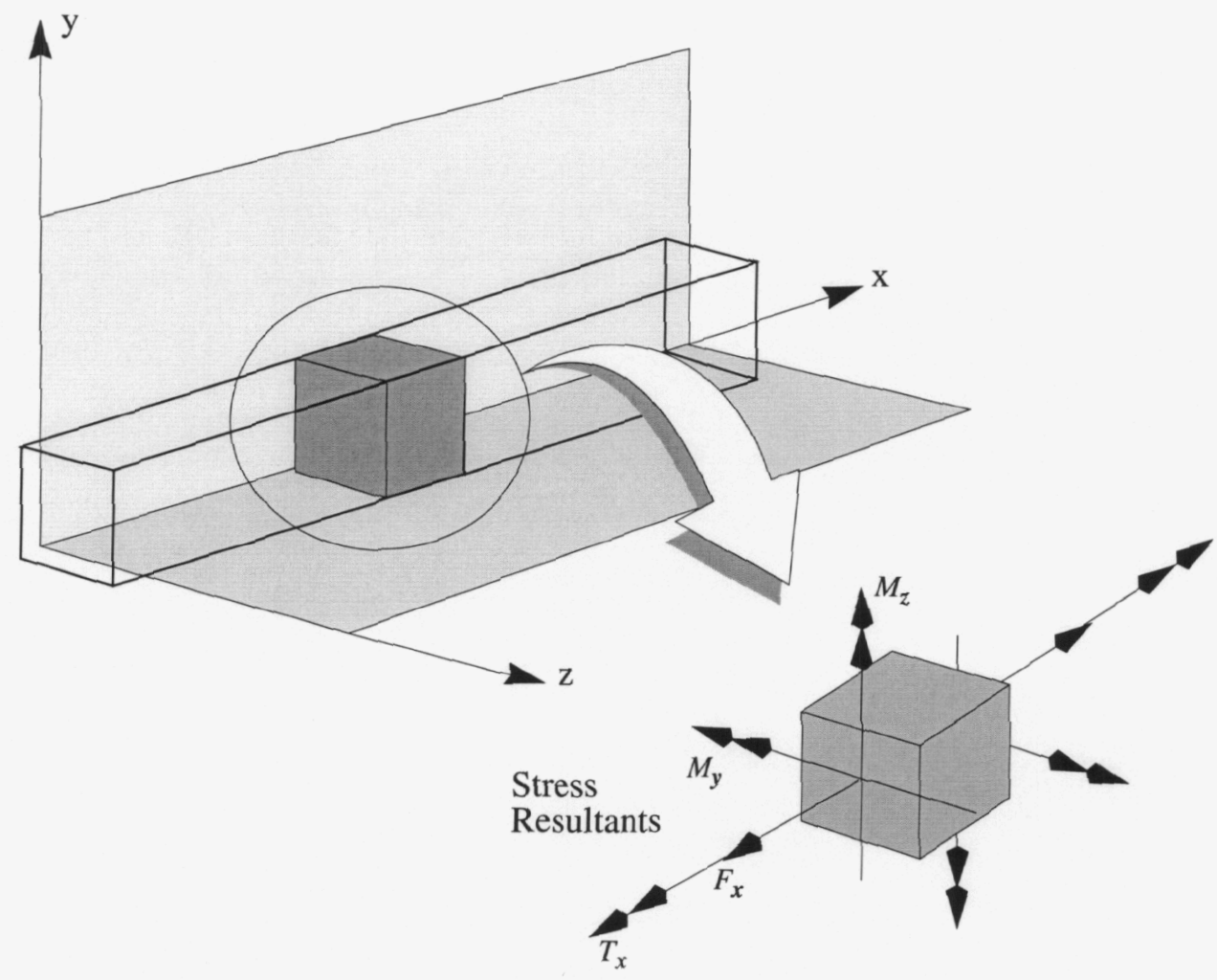

FIGURE 24. Beam stress resultants

Where $A$ is the cross sectional area of the beam and the bars on the stress terms denote the stresses at locations off of the reference axis. For the specialized case of a linear elastic material, the stress-strain constitutive behavior is provided by a classical Hooke's law,

$$
\bar{\varepsilon}_{x}=\frac{1}{E}\left[\bar{\sigma}_{x}-v\left(\bar{\sigma}_{y}+\bar{\sigma}_{z}\right)\right]
$$

For slender beams, the longitudinal fiber stresses are significantly larger than either of the transverse stress components, and Hooke's law can be approximated by,

$$
\bar{\varepsilon}_{x}=\frac{1}{E} \bar{\sigma}_{x}
$$

Combining EQ. 128 and EQ. 122 gives,

$$
\bar{\sigma}_{x}=E \varepsilon_{x}-E \kappa_{y} y-E \kappa_{z} z
$$

Substituting this stress equation into the stress resultant expressions of EQ. 123 to EQ. 125 yields, 


$$
\begin{gathered}
F_{x}=\int_{A}\left(E \varepsilon_{x}-E \kappa_{y} y-E \kappa_{z} z\right) d A \\
M_{y}=-\int_{A}\left(E \varepsilon_{x}-E \kappa_{y} y-E \kappa_{z} z\right) y d A \\
M_{z}=-\int_{A}\left(E \varepsilon_{x}-E \kappa_{y} y-E \kappa_{z} z\right) z d A \\
T_{x}=\int_{A}\left(G \gamma_{x z} y-G \gamma_{x y} z\right) d A
\end{gathered}
$$

For a homogeneous, elastic material, these relationships can be rewritten,

$$
\begin{gathered}
F_{x}=E A \varepsilon_{x}-E \kappa_{y} \int_{A} y d A-E \kappa_{z} \int_{A} z d A \\
M_{y}=-E \varepsilon_{x} \int_{A} y d A+E \kappa_{y} \int_{A} y^{2} d A+E \kappa_{z} \int_{A} z y d A \\
M_{z}=-E \varepsilon_{x} \int_{A} z d A+E \kappa_{y} \int_{A} y z d A+E \kappa_{z} \int_{A} z^{2} d A \\
T_{x}=G \int_{A}\left(\gamma_{x z} y-\gamma_{x y} z\right) d A
\end{gathered}
$$

EQ. 134 to EQ. 137 do not depend on any specific location of the reference axes. For a linear elastic material however, if the reference axes are taken to coincide with the centroidal axes of the beam, the relationships reduce to the familiar strength of materials expressions for simple Bernoulli-Euler beam bending. For example, if the reference axes correspond to the centroidal axes, by definition,

$$
\begin{aligned}
\int_{A} y d A & =0 \\
\int_{A} z d A & =0 \\
\int_{A} y^{2} d A & =I_{y y} \\
\int_{A} z^{2} d A & =I_{z z} \\
\int_{A} x y d A & =0
\end{aligned}
$$

and EQ. 134 through EQ. 137 reduce to the standard expressions

$$
\begin{aligned}
F_{x} & =E A \varepsilon_{x} \\
M_{y} & =E I_{y y} \kappa_{y} \\
M_{z} & =E I_{z z} \kappa_{z} \\
T_{x} & =G J \gamma
\end{aligned}
$$


From EQ. 143 it is evident that if the axial force applied to the beam is zero, the reference axis strain is zero and thus the reference axis (and centroidal axis for this case) is also the neutral axis of the beam. When the reference axes correspond to the centroidal axes the beam axial force is only a function of the reference axis strain and the moments are only a function of the reference axis curvature. In the consideration of material nonlinearity in a subsequent section, it will be found that the uncoupling of the axial stress resultant from the curvature terms and the uncoupling of the moments from the reference axis strain will not generally be achievable because the neutral axis of the beam translates through the beam section as portions of the beam cross section undergo nonlinear material response.

\subsubsection{Beam finite element}

In typical civil engineering applications, the structure consists of a frame or truss system in which a large number of beam elements constitute the structural system. For seismic analyses of typical structures, many regions of the structure may remain entirely in the elastic range while other regions of the structure experience nonlinear material behavior. Most often, the regions of nonlinearity are not known a priori and depend on the complex dynamic transient response of the entire structural system. It is thus desirable to have a structural model which can efficiently and accurately represent both linear and nonlinear behavior. With this concept in mind, a nonlinear beam element has been developed based on a cubic Hermite polynomial displacement field approximation. This element allows adequate representation of linear beam bending with a single element between nodes of the model. This is in contrast to some existing linear beam elements (see for example NIKE3D beam element technology) which require multiple element discretizations of structural elements in order to accurately represent the linear bending characteristics of the beam. Discretization with linear displacement field elements can result in an excessively large number of model degrees of freedom when representing a long-span bridge.

Consider the one dimensional flexural element shown in Figure 25. The element contributions to the internal resisting force vector and instantaneous stiffness matrix can be obtained by consideration of the principal of virtual displacements. For any vector of imposed virtual displacements $\delta d$, the internal work must equal the external work,

$$
\begin{gathered}
\int_{0}^{l}\left[F_{x}\left(x^{\prime}\right) \delta \varepsilon_{x}\left(x^{\prime}\right)+M_{y}\left(x^{\prime}\right) \delta \kappa_{y}\left(x^{\prime}\right)+M_{z}\left(x^{\prime}\right) \delta \kappa_{z}\left(x^{\prime}\right)+T_{x}\left(x^{\prime}\right) \delta \Gamma_{x}\left(x^{\prime}\right)\right] d x^{\prime} \\
=q_{1} \delta d_{1}+q_{2} \delta d_{2}+q_{3} \delta d_{3}+q_{4} \delta d_{4}+q_{5} \delta d_{5}+q_{6} \delta d_{6}+q_{7} \delta d_{7}+ \\
q_{8} \delta d_{8}+q_{9} \delta d_{9}+q_{10} \delta d_{10}+q_{11} \delta d_{11}+q_{12} \delta d_{12} \\
\int_{0}^{l}\left[\left\{F\left(x^{\prime}\right)\right\}^{T}\left\{\delta \varepsilon\left(x^{\prime}\right)\right\}\right] d x^{\prime}=\{q\}^{T}\{\delta d\}
\end{gathered}
$$$$
\text { or }
$$

where 


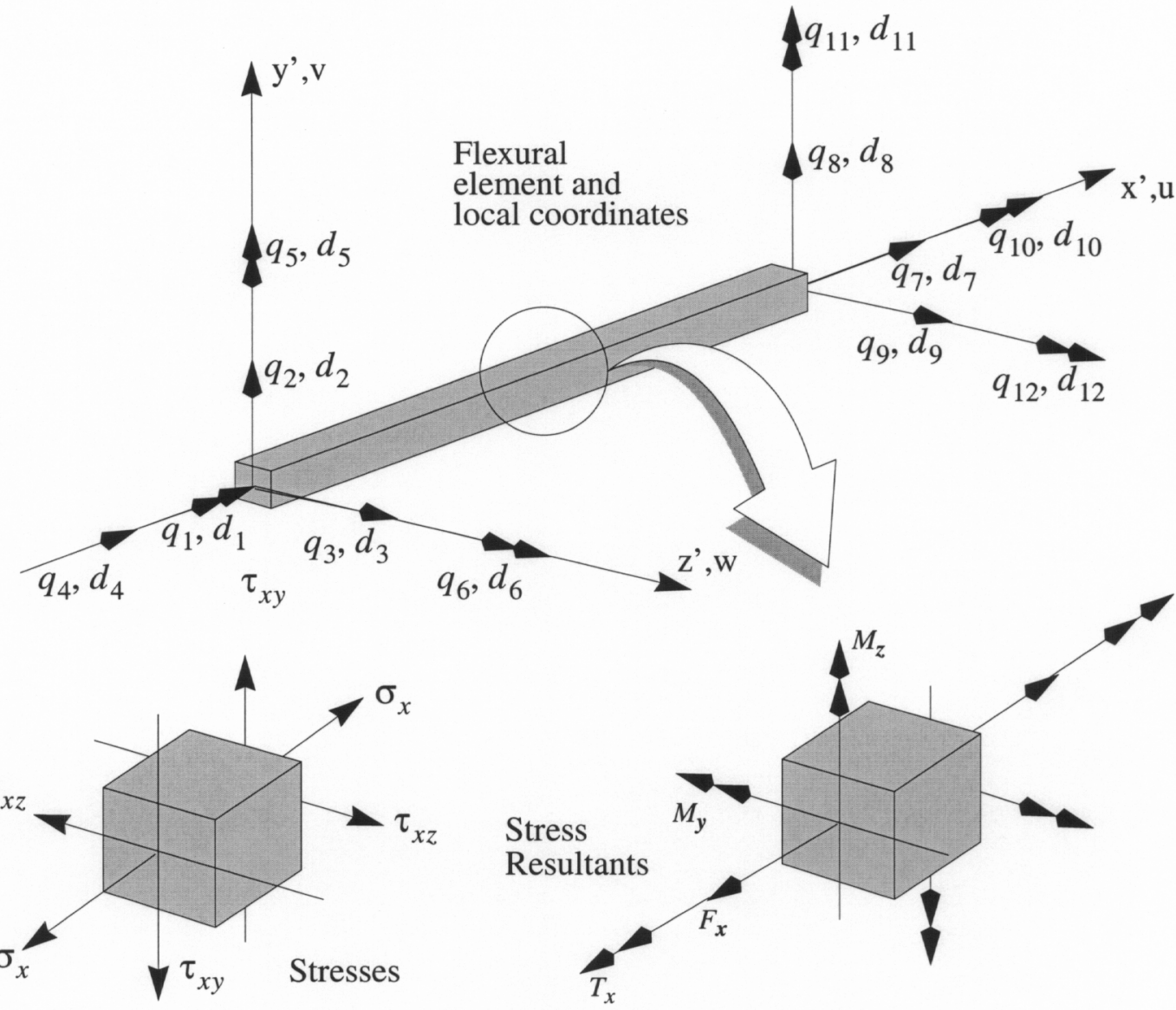

FIGURE 25. 1D flexure element- local coordinate system, stress resultants and active degrees of freedom

$$
\begin{aligned}
& \left\{F\left(x^{\prime}\right)\right\}^{T}=\left[F_{x}\left(x^{\prime}\right) M_{y}\left(x^{\prime}\right) M_{z}\left(x^{\prime}\right) T_{x}\left(x^{\prime}\right)\right] \\
& \left\{\delta \varepsilon\left(x^{\prime}\right)\right\}=\left[\delta \varepsilon_{x} \delta \kappa_{y} \delta \kappa_{z} \delta \Gamma_{x}\right] \\
& \{q\}^{T}=\left[\begin{array}{llllllllllll}
q_{1} & q_{2} & q_{3} & q_{4} & q_{5} & q_{6} & q_{7} & q_{8} & q_{9} & q_{10} & q_{11} & q_{12}
\end{array}\right]
\end{aligned}
$$

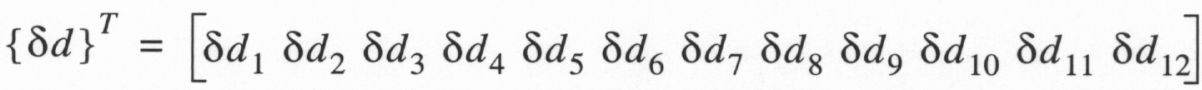

and $\Gamma$ is the twist of the beam about the longitudinal axis. To facilitate the ultimate computation of the element residual and stiffness contributions, a transformation from element to natural coordinates is made. The relationship between element physical and natural coordinates is given by (see Figure 26),

$$
\xi=\frac{2 x}{l}-1
$$

and 

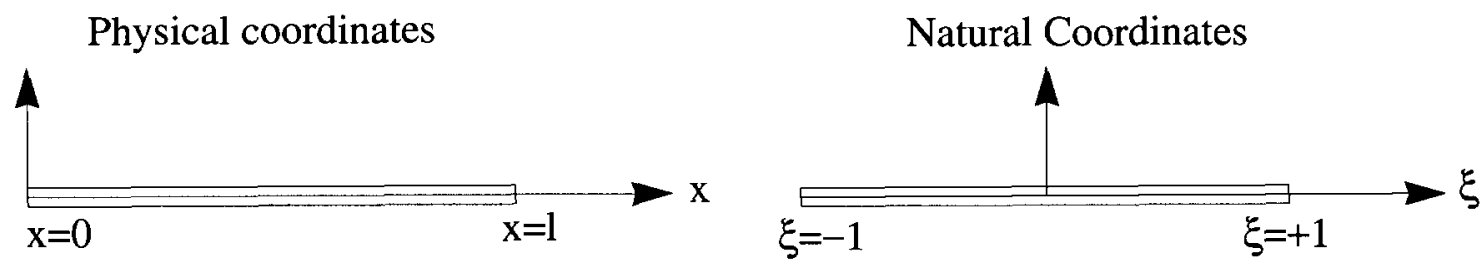

FIGURE 26. Flexural element coordinate systems

$$
d \xi=\frac{2}{l} d x
$$

The transformation to a semi discrete system is made by introduction of the displacement field approximations,

$$
\begin{aligned}
& u=\sum_{i=1}^{2} N_{i}(\xi) u_{i} \\
& v=\sum_{i=1}^{4} M_{i}(\xi) v_{i} \\
& w=\sum_{i=1}^{4} L_{i}(\xi) w_{i} \\
& \theta=\sum_{i=1}^{2} N_{i}(\xi) \theta_{i}
\end{aligned}
$$

For computational expediency, the quadratic terms of EQ. 121 (i.e. $\left(\frac{d v}{d x}\right)^{2}$ and $\left(\frac{d w}{d x}\right)^{2}$ ) will be represented by a lower order approximation (Przemieniecki, 1968). A second order approximation for the slope terms would result if the displacement approximations of EQ. 156 and EQ. 157 were used directly, and the corresponding instantaneous stiffness and residual terms would include high order terms. The approximate expressions for the slope terms are given by,

$$
\begin{gathered}
\frac{d v}{d \xi} \cong \sum_{i=1}^{2} N_{i}^{\prime}(\xi) v_{i} \\
\frac{d w}{d \xi} \cong \sum_{i=1}^{2} N_{i}^{\prime}(\xi) w_{i}
\end{gathered}
$$

In EQ. 155 through EQ. 158, the $u_{i}, v_{i}, w_{i}$ and $\theta_{\imath}$ terms are displacements and rotations at the ends of the element, which are equivalent to the corresponding " $d$ " terms in Figure 25 (for example $v_{1}=d_{2}, v_{2}=d_{6}, v_{3}=d_{8}, v_{4}=d_{12}$ ) and 


$$
\begin{gathered}
N_{1}=\frac{1}{2}(1-\xi) \\
N_{2}=\frac{1}{2}(1+\xi) \\
M_{1}=\frac{1}{4}(1-\xi)^{2}(2+\xi) \\
M_{2}=\frac{l}{8}(\xi+1)(1-\xi)^{2} \\
M_{3}=\frac{1}{4}(\xi+1)^{2}(2-\xi) \\
M_{4}=\frac{l}{8}(\xi+1)^{2}(\xi-1) \\
L_{1}=\frac{1}{4}(1-\xi)^{2}(2+\xi) \\
L_{2}=-\frac{l}{8}(\xi+1)(1-\xi)^{2} \\
L_{3}=\frac{1}{4}(\xi+1)^{2}(2-\xi) \\
L_{4}=-\frac{l}{8}(\xi+1)^{2}(\xi-1)
\end{gathered}
$$

In terms of natural coordinates the element strains can be written,

$$
\varepsilon_{x}=\left(\frac{d u}{d \xi} \frac{d \xi}{d x}\right)+\frac{1}{2}\left(\frac{d v}{d \xi} \frac{d \xi}{d x}\right)^{2}+\frac{1}{2}\left(\frac{d w}{d \xi} \frac{d \xi}{d x}\right)^{2}
$$

or

$$
\varepsilon_{x}=\left(\frac{d u}{d \xi} \frac{2}{l}\right)+\frac{1}{2}\left(\frac{d v}{d \xi} \frac{2}{l}\right)^{2}+\frac{1}{2}\left(\frac{d w}{d \xi} \frac{2}{l}\right)^{2}
$$

similarly,

$$
\begin{gathered}
\kappa_{y}=\frac{d^{2} v}{d \xi^{2}} \frac{4}{l^{2}} \\
\kappa_{z}=\frac{d^{2} w}{d \xi^{2}} \frac{4}{l^{2}} \\
\Gamma=\frac{d \theta}{d \xi} \frac{2}{l}
\end{gathered}
$$

Introducing these strain expressions into the PVD statement of EQ. 147 and converting to natural coordinates yields, 


$$
\begin{array}{r}
\int_{-1}^{1}\left[F _ { x } ( \xi ) \left[\left(\sum_{i=1}^{2} N_{i}^{\prime}(\xi) \delta u_{i} J^{-1}\right)+\left(\sum_{i=1}^{2} N_{i}^{\prime} v_{i} J^{-1}\right)\left(\sum_{i=1}^{2} N_{i}^{\prime} \delta v_{i} J^{-1}\right)\right.\right. \\
\left.+\left(\sum_{i=1}^{2} N_{i}^{\prime} w_{i} J^{-1}\right)\left(\sum_{i=1}^{2} N_{i}^{\prime} \delta w_{i} J^{-1}\right)\right]+M_{y}\left(\sum_{i=1}^{4} M_{i}^{\prime \prime}(\xi) \delta v_{i}\left(J^{-1}\right)^{2}\right) \\
\left.+M_{z}\left(\sum_{i=1}^{4} L_{i}^{\prime \prime}(\xi) \delta w_{i}\left(J^{-1}\right)^{2}\right)+T_{x}\left(\sum_{i=1}^{2} N_{i}^{\prime}(\xi) \delta u_{i}\left(J^{-1}\right)^{2}\right)\right] J d \xi \\
=q_{1} \delta d_{1}+\ldots q_{12} \delta d_{12}
\end{array}
$$

which can be written in matrix form,

$$
\begin{array}{r}
\int_{-1}^{1}\left[F_{x}\left\{\left\{B_{1}\right\}+\left\{B_{1_{G}}(\{d\})\right\}\right\}\{\delta d\}+M_{y}\left\{B_{2}\right\}\{\delta d\}+M_{z}\left\{B_{3}\right\}\{\delta d\}\right. \\
\left.+T_{x}\left\{B_{4}\right\}\{\delta d\}\right] J d \xi=\{q\}^{T}\{\delta d\}
\end{array}
$$

where,

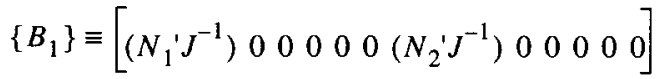

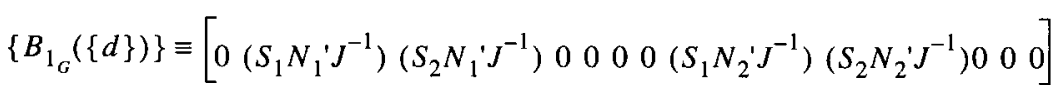

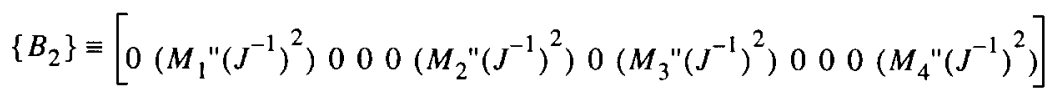

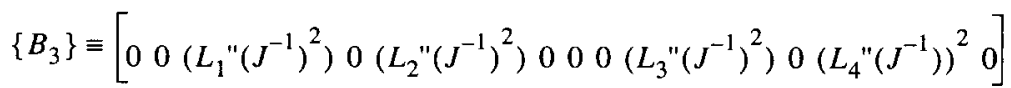

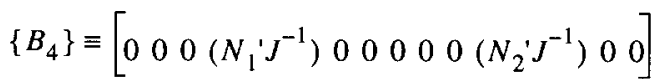

and

$$
\begin{aligned}
& S_{1}=\sum_{i=1}^{2} N_{i}^{\prime}(\xi) v_{i} J^{-1} \\
& S_{2}=\sum_{i=1}^{2} N_{i}^{\prime}(\xi) w_{i} J^{-1}
\end{aligned}
$$

The principal of virtual displacement can then be written,

$$
\int_{-1}^{1}\{F\}^{T}\left[[B]+\left[B_{G}(\{d\})\right]\right]\{\delta d\} J d \xi=\{q\}^{T}\{\delta d\}
$$

Comparing EQ. 185 and EQ. 148, the relationship between strain and displacements is given by

where,

$$
\{\delta \varepsilon\}=\left[[B]+\left[B_{G}(\{d\})\right]\right]\{\delta d\}
$$




$$
\begin{aligned}
& {[B]=} \\
& \text { (EQ 187) } \\
& {\left[\begin{array}{cccccccccccc}
N_{1} J^{-1} & 0 & 0 & 0 & 0 & 0 & N_{2} J^{-1} & 0 & 0 & 0 & 0 & 0 \\
0 & M_{1}^{\prime \prime}\left(J^{-1}\right)^{2} & 0 & 0 & 0 & M_{2}^{\prime \prime}\left(J^{-1}\right)^{2} & 0 & M_{3}{ }^{\prime \prime}\left(J^{-1}\right)^{2} & 0 & 0 & 0 & M_{4}{ }^{\prime \prime}\left(J^{-1}\right)^{2} \\
0 & 0 & L_{1}{ }^{\prime \prime}\left(J^{-1}\right)^{2} & 0 & L_{2}^{\prime \prime}\left(J^{-1}\right)^{2} & 0 & 0 & 0 & L_{3}^{\prime \prime}\left(J^{-1}\right)^{2} & 0 & L_{4}^{\prime \prime}\left(J^{-1}\right)^{2} & 0 \\
0 & 0 & 0 & N_{1} J^{-1} & 0 & 0 & 0 & 0 & 0 & N_{2} J^{-1} & 0 & 0
\end{array}\right]} \\
& {\left[B_{G}(\{d\})\right]=} \\
& \text { (EQ 188) }
\end{aligned}
$$

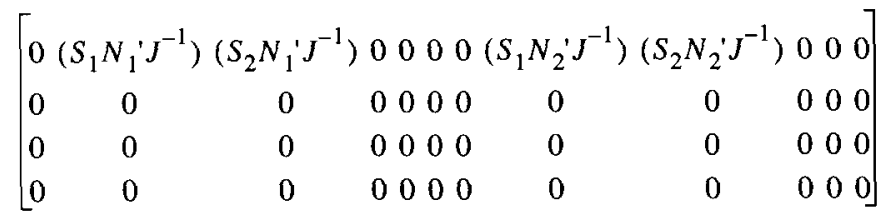

EQ. 185 must hold for any $\{\delta d\}$, thus,

$$
\{q\}=\int_{-1}^{1}\left[[B]+\left[B_{G}(\{d\})\right]\right]^{T}\{F\} J d \xi
$$

EQ. 189 provides the element end forces in the element local coordinate system. By definition, the element instantaneous stiffness matrix consists of the instantaneous rate of change of the element end forces with respect to element end displacements, i.e.

$$
\left[k_{I}\right]=\left[\begin{array}{cccccc}
\left(\frac{\partial q_{1}}{\partial d_{1}}\right)\left(\frac{\partial q_{1}}{\partial d_{2}}\right) & \left(\frac{\partial q_{1}}{\partial d_{3}}\right) & \ldots & \ldots & \left(\frac{\partial q_{1}}{\partial d_{12}}\right) \\
\left(\frac{\partial q_{2}}{\partial d_{1}}\right)\left(\frac{\partial q_{2}}{\partial d_{2}}\right) & \ldots & \ldots & \ldots & \ldots \\
\left(\frac{\partial q_{3}}{\partial d_{1}}\right) & \ldots & \ldots & \ldots & \ldots & \ldots \\
\ldots & \ldots & \ldots & \ldots & \ldots & \left(\frac{\partial q_{10}}{\partial d_{12}}\right) \\
\ldots & \cdots & \ldots & \ldots & \left(\frac{\partial q_{11}}{\partial d_{11}}\right)\left(\frac{\partial q_{11}}{\partial d_{12}}\right) \\
\left(\frac{\partial q_{12}}{\partial d_{1}}\right) & \cdots & \ldots & \left(\frac{\partial q_{12}}{\partial d_{10}}\right)\left(\frac{\partial q_{12}}{\partial d_{11}}\right)\left(\frac{\partial q_{12}}{\partial d_{12}}\right)
\end{array}\right]
$$

EQ. 190 can be written,

$$
\left[k_{I}\right]=\left[\left\{\frac{\partial q}{\partial d_{1}}\right\}\left\{\frac{\partial q}{\partial d_{2}}\right\}\left\{\frac{\partial q}{\partial d_{3}}\right\} \cdots\left\{\frac{\partial q}{\partial d_{12}}\right\}\right]
$$

From EQ. 189 ,

$$
\left\{\frac{\partial q}{\partial d_{1}}\right\}=\int_{-1}^{1}\left[\left[[B]+\left[B_{G}(\{d\})\right]\right]^{T}\left\{\frac{\partial F}{\partial d_{1}}\right\}+\left[\frac{\partial}{\partial d_{1}} B_{G}(\{d\})\right]^{T}\{F\}\right] J d \xi
$$




$$
\left\{\frac{\partial q}{\partial d_{2}}\right\}=\int_{-1}^{1}\left[\left[[B]+\left[B_{G}(\{d\})\right]\right]^{T}\left\{\frac{\partial F}{\partial d_{2}}\right\}+\left[\frac{\partial}{\partial d_{2}} B_{G}(\{d\})\right]^{T}\{F\}\right] J d \xi
$$

similar expressions exist for $d_{3}$ through $d_{12}$, and the element instantaneous stiffness can thus be written,

$$
\begin{aligned}
& {\left[k_{l}\right]=\left[\frac{\partial q}{\partial d}\right]} \\
& =\int_{-1}^{1}\left[\left[[B]+\left[B_{G}(\{d\})\right]\right]^{T}\left[\frac{\partial F}{\partial d}\right]+\left[\left[\frac{\partial}{\partial d_{k}} B_{G}(\{d\})\right]^{T}\{F\}\right]_{k=1,12}\right] J d \xi
\end{aligned}
$$

where

$$
\left[\frac{\partial F}{\partial d}\right]=\left[\begin{array}{ccccc}
\left(\frac{\partial F_{x}}{\partial d_{1}}\right)\left(\frac{\partial F_{x}}{\partial d_{2}}\right) & \ldots & \ldots & \left(\frac{\partial F_{x}}{\partial d_{12}}\right) \\
\left(\frac{\partial M_{y}}{\partial d_{1}}\right) & \ldots & \ldots & \ldots & \ldots \\
\left(\frac{\partial M_{z}}{\partial d_{1}}\right) & \ldots & \ldots & \ldots & \left(\frac{\partial M_{x}}{\partial d_{12}}\right) \\
\left(\frac{\partial T}{\partial d_{1}}\right) & \ldots & \ldots\left(\frac{\partial T}{\partial d_{11}}\right) & \left(\frac{\partial T}{\partial d_{12}}\right)
\end{array}\right]
$$

and

$$
\begin{aligned}
& {\left[\left[\frac{\partial}{\partial d_{k}} B_{G}(\{d\})\right]^{T}\{F\}\right]_{k=1,12}=} \\
& {\left[\left\{\left[\frac{\partial}{\partial d_{1}} B_{G}(\{d\})\right]^{T}\{F\}\right\}\left\{\left[\frac{\partial}{\partial d_{1}} B_{G}(\{d\})\right]^{T}\{F\}\right\} \cdots\left\{\left[\frac{\partial}{\partial d_{12}} B_{G}(\{d\})\right]^{T}\{F\}\right\}\right]}
\end{aligned}
$$

The chain rule of differentiation can be applied to EQ. 195 to yield

$$
\left[\frac{\partial F}{\partial d}\right]=\left[\frac{\partial F}{\partial \varepsilon}\right]\left[\frac{\partial \varepsilon}{\partial d}\right]
$$

where

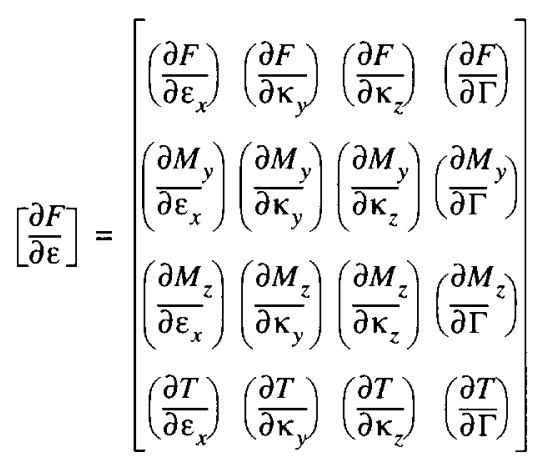

and,

(EQ 196) 


$$
\left[\frac{\partial \varepsilon}{\partial d}\right]=\left[\begin{array}{lllll}
\left(\frac{\partial \varepsilon_{x}}{\partial d_{1}}\right) & \left(\frac{\partial \varepsilon_{x}}{\partial d_{2}}\right) & \ldots & \ldots & \left(\frac{\partial \varepsilon_{x}}{\partial d_{12}}\right) \\
\left(\frac{\partial \kappa_{y}}{\partial d_{1}}\right) & \ldots & \ldots & \ldots & \left(\frac{\partial \kappa_{y}}{\partial d_{12}}\right) \\
\left(\frac{\partial \kappa_{z}}{\partial d_{1}}\right) & \ldots & \ldots & \ldots & \left(\frac{\partial \kappa_{z}}{\partial d_{12}}\right) \\
\left(\frac{\partial \Gamma}{\partial d_{1}}\right) & \ldots & \ldots & \left(\frac{\partial \Gamma}{\partial d_{11}}\right) & \left(\frac{\partial \Gamma}{\partial d_{12}}\right)
\end{array}\right]
$$

(EQ 199)

Comparison of EQ. 199 and EQ. 186 shows that EQ. 199 can be written,

$$
\left[\frac{\partial \varepsilon}{\partial d}\right]=\left[[B]+\left[B_{G}(\{d\})\right]\right]
$$

The instantaneous stiffness matrix given by EQ. 194 can then be written,

$$
\left[k_{I}\right]=\left[\frac{\partial q}{\partial d}\right]=\int_{-1}^{1}\left[\left[[B]+\left[B_{G}(\{d\})\right]\right]^{T}\left[\frac{\partial F}{\partial \varepsilon}\right]\left[[B]+\left[B_{G}(\{d\})\right]\right]+\left[\left[\frac{\partial}{\partial d_{k}} B_{G}(\{d\})\right]^{T}\{F\}\right]_{k=1,12}\right]_{J d \xi}
$$

or

$$
\left[k_{I}\right]=\left[k_{i}\right]+\left[k_{G_{1}}\right]+\left[k_{G_{2}}\right]+\left[k_{G_{3}}\right]+\left[k_{G_{4}}\right]
$$

and

$$
\begin{gathered}
{\left[k_{i}\right]=\int_{-1}^{1}[B]^{T}\left[\frac{\partial F}{\partial \varepsilon}\right][B] J d \xi} \\
{\left[k_{G_{1}}\right]=\int_{-1}^{1}\left[B_{G}(\{d\})\right]^{T}\left[\frac{\partial F}{\partial \varepsilon}\right][B] J d \xi} \\
{\left[k_{G_{2}}\right]=\int_{-1}^{1}[B]^{T}\left[\frac{\partial F}{\partial \varepsilon}\right]\left[B_{G}(\{d\})\right] J d \xi} \\
{\left[k_{G_{3}}\right]=\int_{-1}^{1}\left[B_{G}(\{d\})\right]^{T}\left[\frac{\partial F}{\partial \varepsilon}\right]\left[B_{G}(\{d\})\right] J d \xi} \\
{\left[k_{G_{4}}\right]=\int_{-1}^{1}\left[\left[\frac{\partial}{\partial d_{k}} B_{G}(\{d\})\right]^{T}\{F\}\right]_{k=1,12} J d \xi}
\end{gathered}
$$

In the updated corotational coordinate system developed for the beam element (section 4.0.1), the element end displacements in the updated coordinate system are identically zero (see Figure 27 below). Thus, with the simple slope approximation given by EQ. 159 and EQ. 160, the matrix $\left[B_{G}(\{d\})\right]$ vanishes, and the instantaneous stiffness terms 
$\left[k_{G_{1}}\right],\left[k_{G_{2}}\right]$, and $\left[k_{G_{3}}\right]$ are identically zero. For the updated corotational system, the ele-

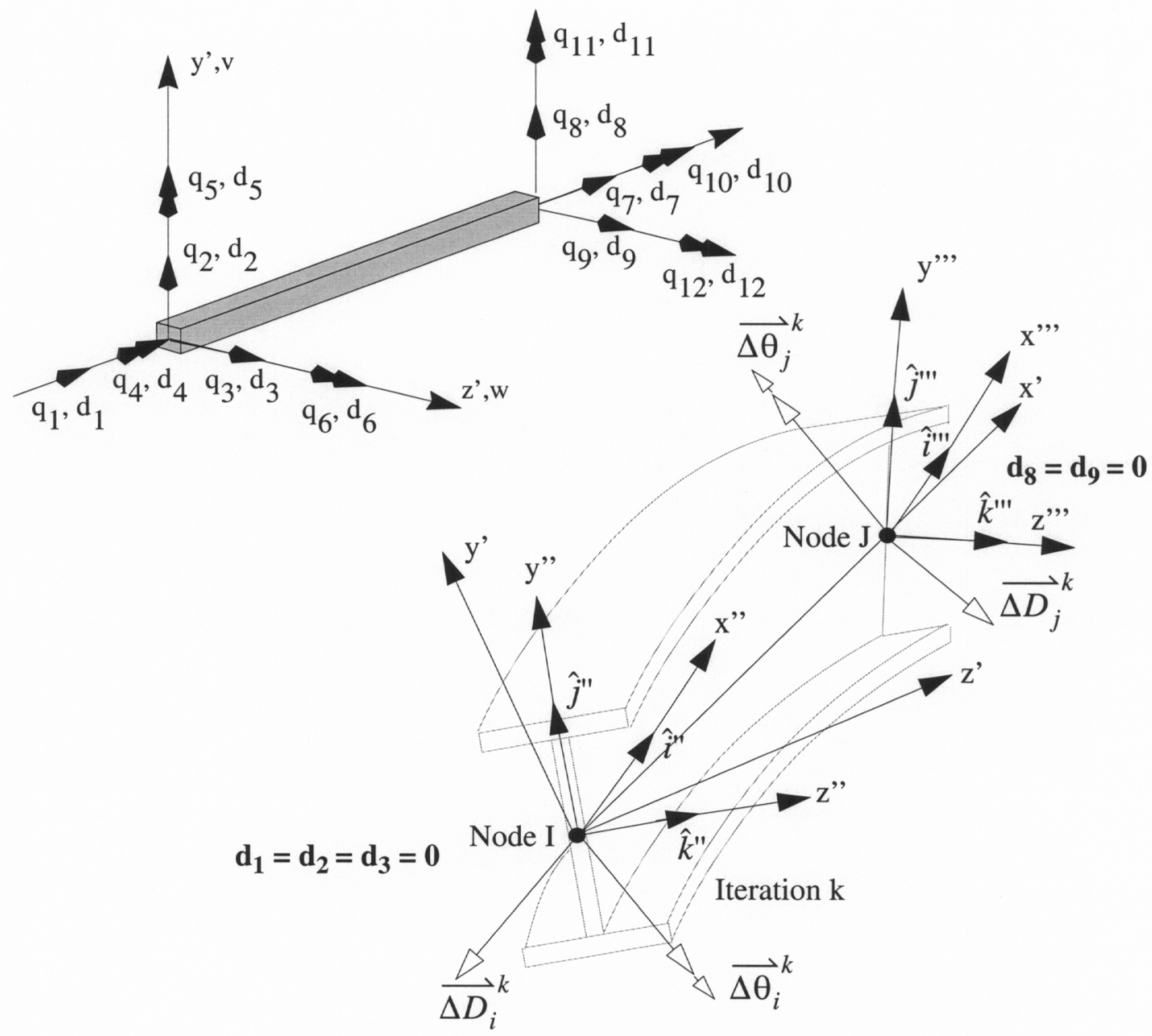

FIGURE 27. Flexural element and convected corrotational coordinate system

ment residual and instantaneous stiffness contributions are thus given by,

$$
\begin{gathered}
\{q\}=\int_{-1}^{1}[B]^{T}\{F\} J d \xi \\
{\left[k_{I}\right]=\int_{-1}^{1}[B]^{T}\left[\frac{\partial F}{\partial \varepsilon}\right][B] J d \xi+\int_{-1}^{1}\left[\left[\frac{\partial}{\partial d_{k}} B_{G}(\{d\})\right]^{T}\{F\}\right]_{k=1,12} J d \xi}
\end{gathered}
$$

Up to this point in the development, the nonlinear constitutive behavior of the element material has been left completely arbitrary. If the material is linear elastic, the constitutive matrix defining the rate of change of stress resultants with respect to strains (EQ. 198) reduces to a very simple form for the case in which the reference axes correspond to the beam centroidal axes. For this case, the constitutive matrix reduces to 


$$
\left[\frac{\partial F}{\partial \varepsilon}\right]=\left[\begin{array}{ll}
\left(\frac{\partial F}{\partial \varepsilon_{x}}\right) & \left(\frac{\partial F}{\partial \kappa_{y}}\right)\left(\frac{\partial F}{\partial \kappa_{z}}\right)\left(\frac{\partial F}{\partial \Gamma}\right) \\
\left(\frac{\partial M_{y}}{\partial \varepsilon_{x}}\right)\left(\frac{\partial M_{y}}{\partial \kappa_{y}}\right)\left(\frac{\partial M_{y}}{\partial \kappa_{z}}\right)\left(\frac{\partial M_{y}}{\partial \Gamma}\right) \\
\left(\frac{\partial M_{z}}{\partial \varepsilon_{x}}\right)\left(\frac{\partial M_{z}}{\partial \kappa_{y}}\right)\left(\frac{\partial M_{z}}{\partial \kappa_{z}}\right)\left(\frac{\partial M_{z}}{\partial \Gamma}\right) \\
\left(\frac{\partial T}{\partial \varepsilon_{x}}\right)\left(\frac{\partial T}{\partial \kappa_{y}}\right)\left(\frac{\partial T}{\partial \kappa_{z}}\right)\left(\frac{\partial T}{\partial \Gamma}\right)
\end{array}\right]=\left[\begin{array}{cccc}
0 & E I_{y y} & 0 & 0 \\
0 & 0 & E I_{z z} & 0 \\
0 & 0 & 0 & G J
\end{array}\right]
$$

\subsubsection{Finite fiber beam cross section}

To accommodate general nonlinear material behavior in the beam element, the beam cross section will be subdivided into a number of finite fibers ${ }^{1}$ as indicated in Figure 28 , and the stress-strain relationship for the materials of the beam will be defined separately for each finite fiber.

Henceforth in this development, the reference axes (i.e. the $y$ and $z$ axes in Figure 28) will be assumed to correspond to the centroidal axes of the beam cross section.

The force resultants of the beam can now be defined based on the finite fibers,

$$
\begin{gathered}
F_{x}=\sum_{i=1}^{N F I B R} \sigma_{x_{i}} A_{i} \\
M_{y}=-\sum_{i=1}^{N F I B R} \sigma_{x_{i}} y_{i} A_{i} \\
M_{z}=-\sum_{i=1}^{N F I B R} \sigma_{x_{i}} z_{i} A_{i} \\
T_{x}=G J \Gamma
\end{gathered}
$$

The constitutive matrix of the beam can be determined by differentiation of the relationships given in EQ. 211 through EQ. 214. The constitutive relationship for the axial force resultant is given by,

$$
\frac{\partial F_{x}}{\partial \varepsilon_{x}}=\sum_{i=1}^{N F I B R} \frac{d \sigma_{x_{i}}}{d \bar{\varepsilon}_{x_{i}}}\left(\frac{\partial \bar{\varepsilon}_{x_{i}}}{\partial \varepsilon_{x_{i}}}\right) A_{i}
$$

1. The term "finite" as used here refers to the finite dimensions of each defined fiber section, thus distinguishing from the typical connotation of an infinitesimal dimension fiber. 


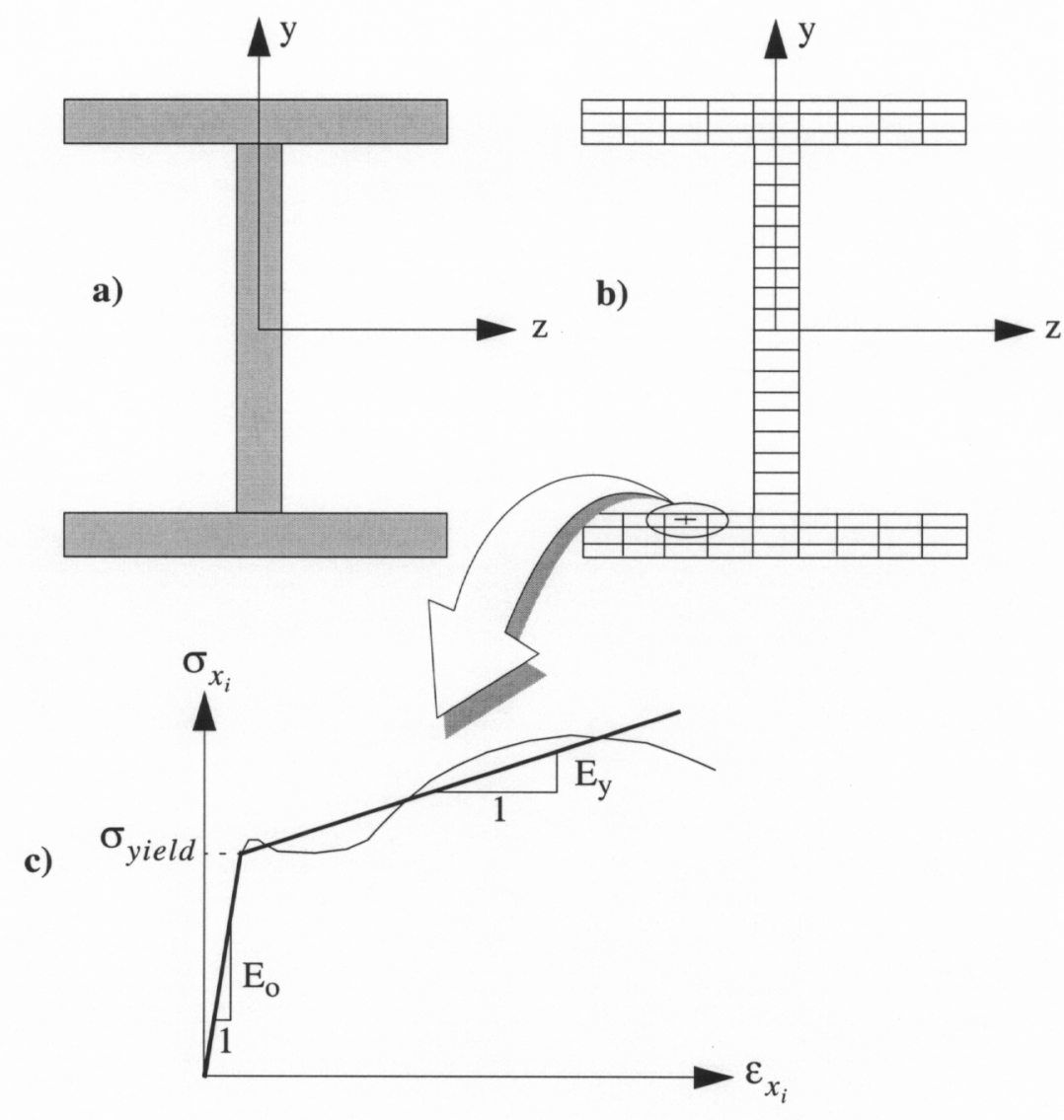

FIGURE 28. Subdivision of beam cross section into finite fibers. (a) Wide flange beam cross section; (b) finite fibers for the cross section; (c) material constitutive behavior at a finite fiber cross section.

from EQ. 122, $\frac{\partial \bar{\varepsilon}_{x}}{\partial \varepsilon_{x}}=1$, and EQ. 215 becomes

$$
\frac{\partial F_{x}}{\partial \varepsilon_{x}}=\sum_{i=1}^{N F I B R} \frac{d \sigma_{x_{i}}}{d \bar{\varepsilon}_{x_{i}}} A_{i}
$$

similarly,

$$
\frac{\partial F_{x}}{\partial \kappa_{y}}=\sum_{i=1}^{N F I B R} \frac{d \sigma_{x_{i}}}{d \bar{\varepsilon}_{x_{i}}}\left(\frac{\partial \bar{\varepsilon}_{x_{i}}}{\partial \kappa_{y}}\right) A_{i}
$$

from EQ. 122, $\frac{\partial \bar{\varepsilon}_{x}}{\partial \kappa_{y}}=-y$ and

$$
\frac{\partial F_{x}}{\partial \kappa_{y}}=-\sum_{i=1}^{N F I B R} \frac{d \sigma_{x_{i}}}{d \bar{\varepsilon}_{x_{i}}} y_{i} A_{i}
$$

and 


$$
\frac{\partial F_{x}}{\partial \kappa_{z}}=\sum_{i=1}^{N F I B R} \frac{d \sigma_{x_{i}}}{d \bar{\varepsilon}_{x_{i}}}\left(\frac{\partial \bar{\varepsilon}_{x_{i}}}{\partial \kappa_{z}}\right) A_{i}
$$

from EQ. 122, $\frac{\partial \bar{\varepsilon}_{x}}{\partial \kappa_{y}}=-z$ thus

and

$$
\frac{\partial F_{x}}{\partial \kappa_{z}}=-\sum_{i=1}^{N F I B R} \frac{d \sigma_{x_{i}}}{d \bar{\varepsilon}_{x_{i}}} z_{i} A_{i}
$$

Similarly,

$$
\frac{\partial F_{x}}{\partial \Gamma}=0
$$

$$
\begin{gathered}
\frac{\partial M_{y}}{\partial \varepsilon_{x}}=-\sum_{i=1}^{N F I B R} \frac{d \sigma_{x}}{d \bar{\varepsilon}_{x}}\left(\frac{\partial \bar{\varepsilon}_{x}}{\partial \varepsilon_{x}}\right) y_{i} A_{i} \\
\frac{\partial M_{y}}{\partial \kappa_{y}}=-\sum_{i=1}^{N F I B R} \frac{d \sigma_{x}}{d \bar{\varepsilon}_{x}}\left(\frac{\partial \bar{\varepsilon}_{x}}{\partial \kappa_{y}}\right) y_{i} A_{i} \\
\frac{\partial M_{y}}{\partial \kappa_{y}}=-\sum_{i=1}^{N F I B R} \frac{d \sigma_{x}}{d \bar{\varepsilon}_{x}}\left(\frac{\partial \bar{\varepsilon}_{x}}{\partial \kappa_{z}}\right) y_{i} A_{i} \\
\frac{\partial M_{y}}{\partial \Gamma}=0
\end{gathered}
$$

EQ. 222 through EQ. 225 can be rewritten

$$
\begin{gathered}
\frac{\partial M_{y}}{\partial \varepsilon_{x}}=-\sum_{i=1}^{N F I B R} \frac{d \sigma_{x}}{d \bar{\varepsilon}_{x}} y_{i} A_{i} \\
\frac{\partial M_{y}}{\partial \kappa_{y}}=\sum_{i=1}^{N F I B R} \frac{d \sigma_{x}}{d \bar{\varepsilon}_{x}} y_{i}^{2} A_{i} \\
\frac{\partial M_{y}}{\partial \kappa_{z}}=\sum_{i=1}^{N F I B R} \frac{d \sigma_{x}}{d \bar{\varepsilon}_{x}} y_{i} z_{i} A_{i} \\
\frac{\partial M_{y}}{\partial \Gamma}=0
\end{gathered}
$$

Similarly,

$$
\frac{\partial M_{z}}{\partial \varepsilon_{x}}=-\sum_{i=1}^{N F I B R} \frac{d \sigma_{x}}{d \bar{\varepsilon}_{x}}\left(\frac{\partial \bar{\varepsilon}_{x}}{\partial \varepsilon_{x}}\right) z_{i} A_{i}
$$




$$
\begin{gathered}
\frac{\partial M_{z}}{\partial \kappa_{y}}=-\sum_{i=1}^{N F I B R} \frac{d \sigma_{x}}{d \bar{\varepsilon}_{x}}\left(\frac{\partial \bar{\varepsilon}_{x}}{\partial \kappa_{y}}\right) z_{i} A_{i} \\
\frac{\partial M_{z}}{\partial \kappa_{z}}=-\sum_{i=1}^{N F I B R} \frac{d \sigma_{x}}{d \bar{\varepsilon}_{x}}\left(\frac{\partial \bar{\varepsilon}_{x}}{\partial \kappa_{z}}\right) z_{i} A_{i} \\
\partial M_{y}=0 \\
\frac{\partial \Gamma}{}=0
\end{gathered}
$$

and EQ. 230 through EQ. 233 can be written,

$$
\begin{gathered}
\frac{\partial M_{z}}{\partial \varepsilon_{x}}=-\sum_{i=1}^{N F I B R} \frac{d \sigma_{x}}{d \bar{\varepsilon}_{x}} z_{i} A_{i} \\
\frac{\partial M_{z}}{\partial \kappa_{y}}=\sum_{i=1}^{N F I B R} \frac{d \sigma_{x}}{d \bar{\varepsilon}_{x}} y_{i} z_{i} A_{i} \\
\frac{\partial M_{z}}{\partial \kappa_{z}}=\sum_{i=1}^{N F I B R} \frac{d \sigma_{x}}{d \bar{\varepsilon}_{x}} z_{i}^{2} A_{i} \\
\frac{\partial M_{y}}{\partial \Gamma}=0
\end{gathered}
$$

\subsubsection{Elasto-plastic constitutive model}

The basic material which will be represented here is a classical elasto-plastic model with kinematic hardening. For this model, the rate of change of stress with respect to strain is linear with the slope determined by whether or not the finite fiber is in a yield condition. For the elasto-plastic material, the rate of change of stress with respect to strain is given simply by,

$$
\frac{d \sigma_{x_{i}}}{d \bar{\varepsilon}_{x_{i}}}=E_{0}
$$

when $\sigma_{x_{i}}<\sigma_{y}$, and

$$
\frac{d \sigma_{x_{i}}}{d \bar{\varepsilon}_{x_{i}}}=E_{y}
$$

when $\sigma_{x_{i}} \geq \sigma_{y}$. The relationships in EQ. 238 and EQ. 239 can simply be written

$$
\frac{d \sigma_{x_{i}}}{d \bar{\varepsilon}_{x_{i}}}=E\left(\varepsilon_{i}\right)
$$

where $E\left(\varepsilon_{i}\right)=E_{0}$ when $\sigma_{x_{i}}<\sigma_{y}$ and $E\left(\varepsilon_{i}\right)=E_{y}$ when $\sigma_{x_{i}} \geq \sigma_{y}$. 


\subsubsection{Implementation of the finite fiber elastoplastic element}

The Newton-Raphson based incremental, iterative global solution algorithm requires the element contributions to the global residual vector and instantaneous stiffness matrix (see and). Based on the developments in the previous sections, the components necessary for development of the beam element residual and instantaneous stiffness matrix are now available. The element end forces in the element updated corotational coordinate system are given by EQ. 208,

$$
\{q\}=\int_{-1}^{1}[B]^{T}\{F\} J d \xi
$$

where, from EQ. 187,

$$
\begin{aligned}
& {[B]=} \\
& {\left[\begin{array}{cccccccccccc}
N_{1} J^{-1} & 0 & 0 & 0 & 0 & 0 & N_{2} J^{-1} & 0 & 0 & 0 & 0 & 0 \\
0 & M_{1}{ }^{\prime \prime}\left(J^{-1}\right)^{2} & 0 & 0 & 0 & M_{2}{ }^{\prime \prime}\left(J^{-1}\right)^{2} & 0 & M_{3}{ }^{\prime \prime}\left(J^{-1}\right)^{2} & 0 & 0 & 0 & M_{4}{ }^{\prime \prime}\left(J^{-1}\right)^{2} \\
0 & 0 & L_{1}{ }^{\prime \prime}\left(J^{-1}\right)^{2} & 0 & L_{2}^{\prime \prime}\left(J^{-1}\right)^{2} & 0 & 0 & 0 & L_{3}^{\prime \prime}\left(J^{-1}\right)^{2} & 0 & L_{4}^{\prime \prime}\left(J^{-1}\right)^{2} & 0 \\
0 & 0 & 0 & N_{1} J^{-1} & 0 & 0 & 0 & 0 & 0 & N_{2} J^{-1} & 0 & 0
\end{array}\right]}
\end{aligned}
$$

The element contribution, for element $i$, to the global resisting force vector is then found from the simple coordinate transformation

$$
\{Q\}_{i}=[T]^{T} \int_{-1}^{1}[B]^{T}\{F\} J d \xi
$$

where $[T]^{T}$ is the transformation matrix between the current corrotational coordinate system for the element and the global coordinate system (see).

The element instantaneous stiffness matrix is provided by EQ. 209,

$$
\left[k_{I}\right]=\int_{-1}^{1}[B]^{T}\left[\frac{\partial F}{\partial \varepsilon}\right][B] J d \xi+\int_{-1}^{1}\left[\left[\frac{\partial}{\partial d_{k}} B_{G}(\{d\})\right]^{T}\{F\}\right]_{k=1,12} J d \xi
$$

where, 


$$
\begin{aligned}
& {\left[B_{G}(\{d\})\right]=} \\
& {\left[\begin{array}{llllllllllll}
0 & \left(S_{1} N_{1}^{\prime} J^{-1}\right) & \left(S_{2} N_{1}^{\prime} J^{-1}\right) & 0 & 0 & 0 & 0 & \left(S_{1} N_{2}^{\prime} J^{-1}\right) & \left(S_{2} N_{2} J^{-1}\right) & 0 & 0 & 0 \\
0 & 0 & 0 & 0 & 0 & 0 & 0 & 0 & 0 & 0 & 0 & 0 \\
0 & 0 & 0 & 0 & 0 & 0 & 0 & 0 & 0 & 0 & 0 & 0 \\
0 & 0 & 0 & 0 & 0 & 0 & 0 & 0 & 0 & 0 & 0 & 0
\end{array}\right]}
\end{aligned}
$$

and from EQ. 198 and EQ. 216 through EQ. 237 the symmetric constitutive matrix is given by,

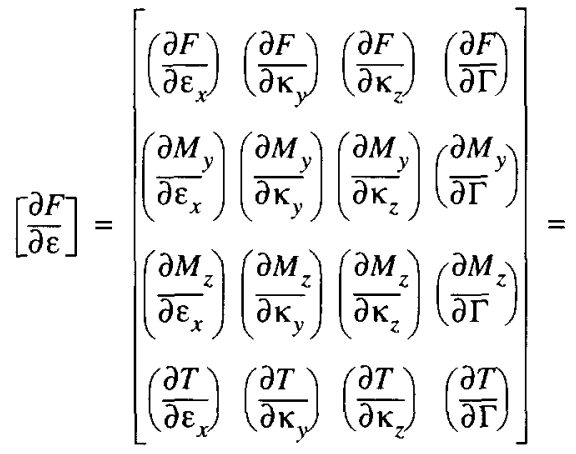

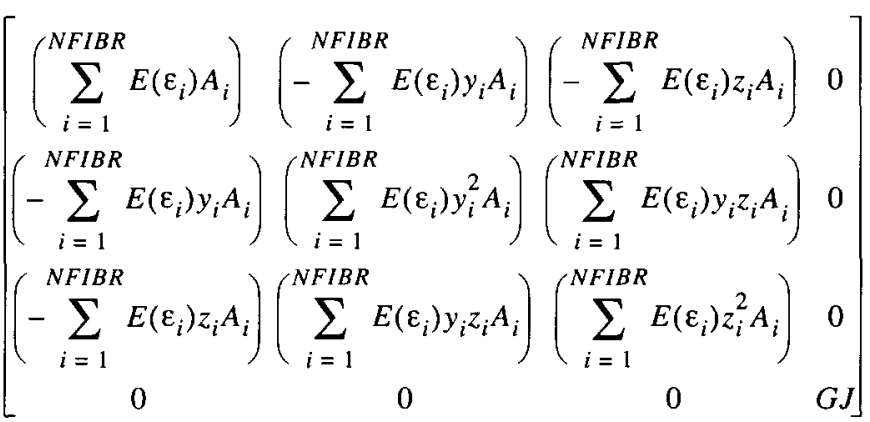

The element contribution to the global instantaneous stiffness matrix is found from the simple coordinate transformation between element corotational and global coordinates,

$$
[K]_{i}=[T]^{T}\left(\int_{-1}^{1}[B]^{T}\left[\frac{\partial F}{\partial \varepsilon}\right][B] J d \xi+\int_{-1}^{1}\left[\left[\frac{\partial}{\partial d_{k}} B_{G}(\{d\})\right]^{T}\{F\}\right]_{k=1,12} J d \xi\right)[T] \quad(\mathrm{EQ} 243)
$$

The integrations indicated in EQ. 243 are performed using a quadrature numerical integration. The well known Gaussian quadrature provides the most economical quadrature rule and for an n-point Gaussian integration rule, a polynomial of degree $2 n-1$ is exactly integrated. However, for the nonlinear beam element developed here an alternative quadrature approach is employed. The alternative approach is based on Lobatto quadrature formulas (Hildebrand, 1956). The distinction of the Lobatto quadrature is that the quadrature points include the extreme ends of the integration interval. This allows capture of the initiation of inelastic action which occurs at the ends of the beam elements, something which Gaussian quadrature will generally miss because the Gauss points are interior to the element. For Lobatto integration, an n-point rule will exactly integrate a polynomial of order $2 \mathrm{n}-3$. For the element integrations indicated in EQ. 243 and EQ. 241, the highest order terms are quadratic in $\xi$, (i.e. order $\left(\xi^{2}\right)$ ), thus a three point Lobatto integration will provide exact 
integration. The Lobatto quadrature points and corresponding weights are shown in Figure 29.

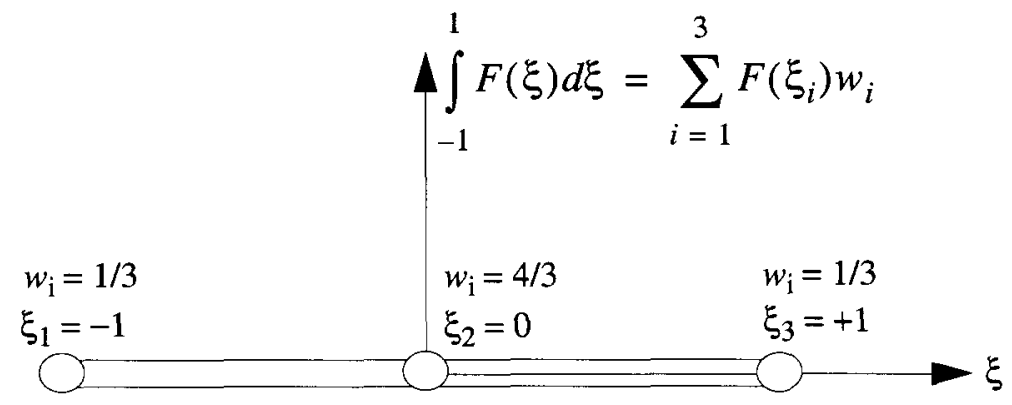

FIGURE 29. Lobatto quadrature points and weights 


\subsection{Representation of steel structure plasticity}

A simple elasto-plastic, linear kinematic hardening model is utilized ro represent steel member elements in the NEVADA program. A general stress evaluation and stress-strain relationship can be developed for the uniaxial plasticity model which can be utilized in all of the elasto-plastic elements.

The classical bilinear plasticity model with kinematic hardening is shown in Figure 30. In the evaluation of element nodal force vectors and instantaneous stiffness matrices, it is necessary to carry out state determinations in which the element stresses at a given equilibrium iteration are evaluated for the element force and stiffness calculations and the instantaneous stress-strain relationship is obtained for the element stiffness determination.

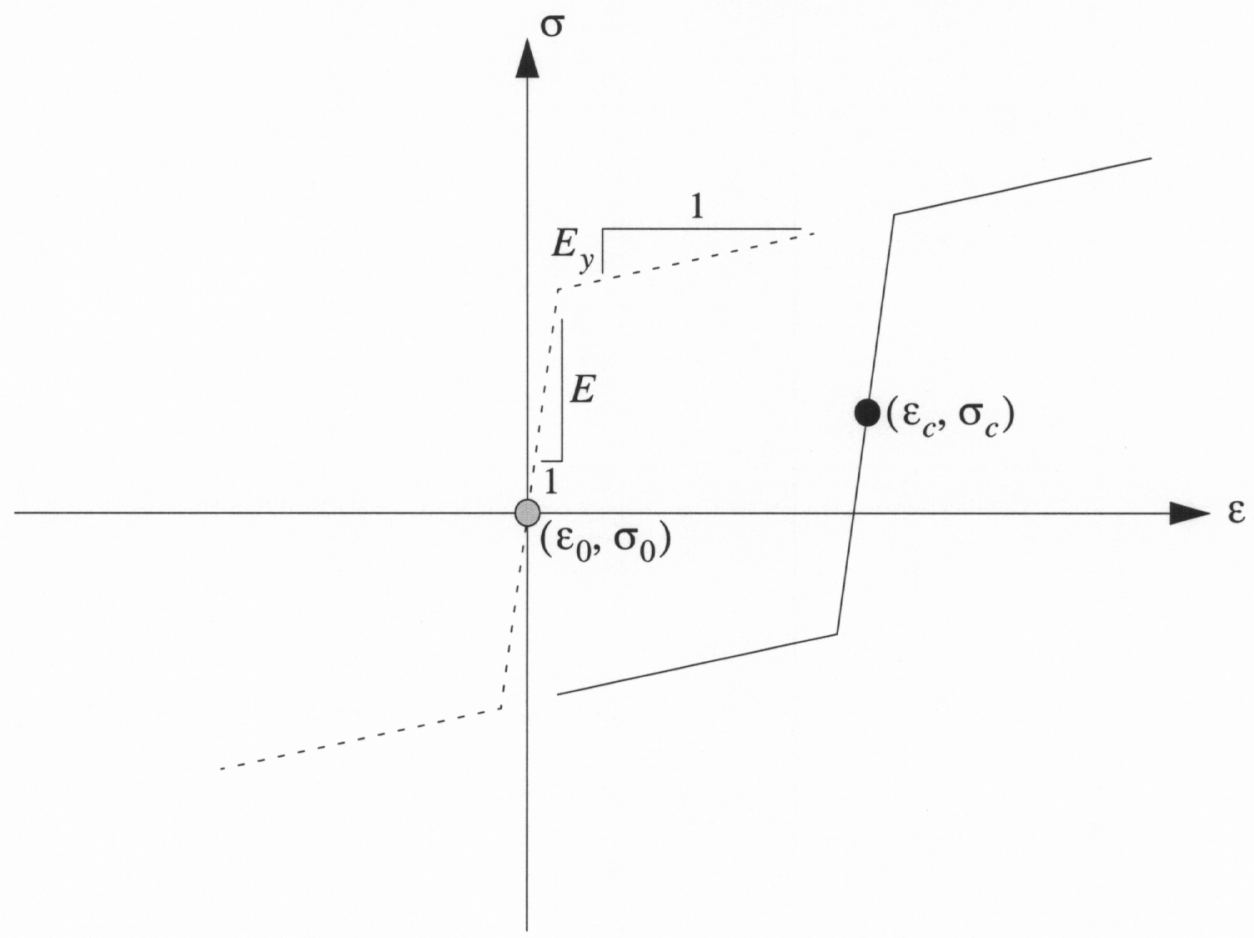

FIGURE 30. Elasto-plastic model with linear kinematic hardening

To evaluate the current state of stress in the incremental-iterative solution procedure, a path independent stress integration procedure will be employed. In the most general form, this relationship can be stated

$$
\sigma_{k}^{n}=\sigma^{n-1}+\int_{\varepsilon^{n-1}}^{\varepsilon_{k}^{n}} \frac{d \sigma}{d \varepsilon} d \varepsilon
$$

In EQ. 244, $n$ refers to the current load step number, $k$ refers to the equilibrium iteration number in the current load step $n, \varepsilon^{n-1}$ is the converged strain at the end of the previous 
load step and $\varepsilon_{k}^{n}$ is the current strain obtained from the displacement field for iteration $k$. EQ. 244 provides the stress value for the current configuration based on an integration of the stress-strain relationship between the last fully converged state and the current displacement configuration. Performing the integration from the last fully converged state rather than the last iteration state ${ }^{1}$ ensures that the algorithm will be path independent.

In the bilinear model, the integration of EQ. 244 is readily accomplished if a number of simple rules are adopted to integrate around yield points. In Figure $30,\left(\varepsilon_{c}, \sigma_{c}\right)$ denotes the current location of the center of the yield region. When performing the integration of EQ. 244 a number of possible cases must be considered as shown in Figure 31. In case I, the last converged state resides within the elastic region and the current configuration for equilibrium iteration $\mathrm{k}$ also lies within the elastic region. In Case II the last converged state is within the yield region and the current configuration lies outside the yield limits. In Case III the last equilibrium configuration lies on the upper or lower yield surface and after load reversal, the new configuration lies either in the elastic range or past the opposite yield point (i.e reverse yielding in a very large load step). In Case IV, yielding continues in the same direction with growth of the yield surface.

To facilitate the development of a simple formula for the stress integration, the parameters $\lambda$ and $\eta$ will be defined (Figure 32 ). These parameters are defined by

$$
\begin{aligned}
\lambda & =\frac{\varepsilon_{y}}{2} \\
\eta & =\frac{E}{E_{y}}
\end{aligned}
$$

In EQ. $245 \varepsilon_{y}$ is the initial yield strain and $\lambda$ represents the half width of the yield region. The parameter $\eta$ is the ratio of the stress-strain slope in the linear region to the stressstrain slope in the inelastic regime.

Consider first the stress integration from point 1 to point 2 in Figure 33. Letting the total strain increment from point 1 to point 2 be denoted by $\Delta \varepsilon$ where

$$
\Delta \varepsilon=\varepsilon_{k}^{n}-\varepsilon^{n-1}
$$

and this strain increment would be obtained from the displacement field in a finite element analysis. The stress increment is given by,

$$
\Delta \sigma=\sigma_{k}^{n}-\sigma^{n-1}=E_{y}\left[\Delta \varepsilon-\left(\varepsilon_{c}+\lambda-\varepsilon^{n-1}\right)\right]+E\left(\varepsilon_{c}+\lambda-\varepsilon^{n-1}\right)
$$

1. For example, performing the integration $\sigma_{k}^{n}=\sigma_{k-1}^{n}+\int_{\varepsilon_{k-1}^{n}}^{\varepsilon_{k}^{n}} \frac{d \sigma}{d \varepsilon} d \varepsilon$ would not preserve path independence since the integration from converged state to converged state would rely on intermediate, unconverged configurations and would introduce path dependency along a nonequilibrium path 

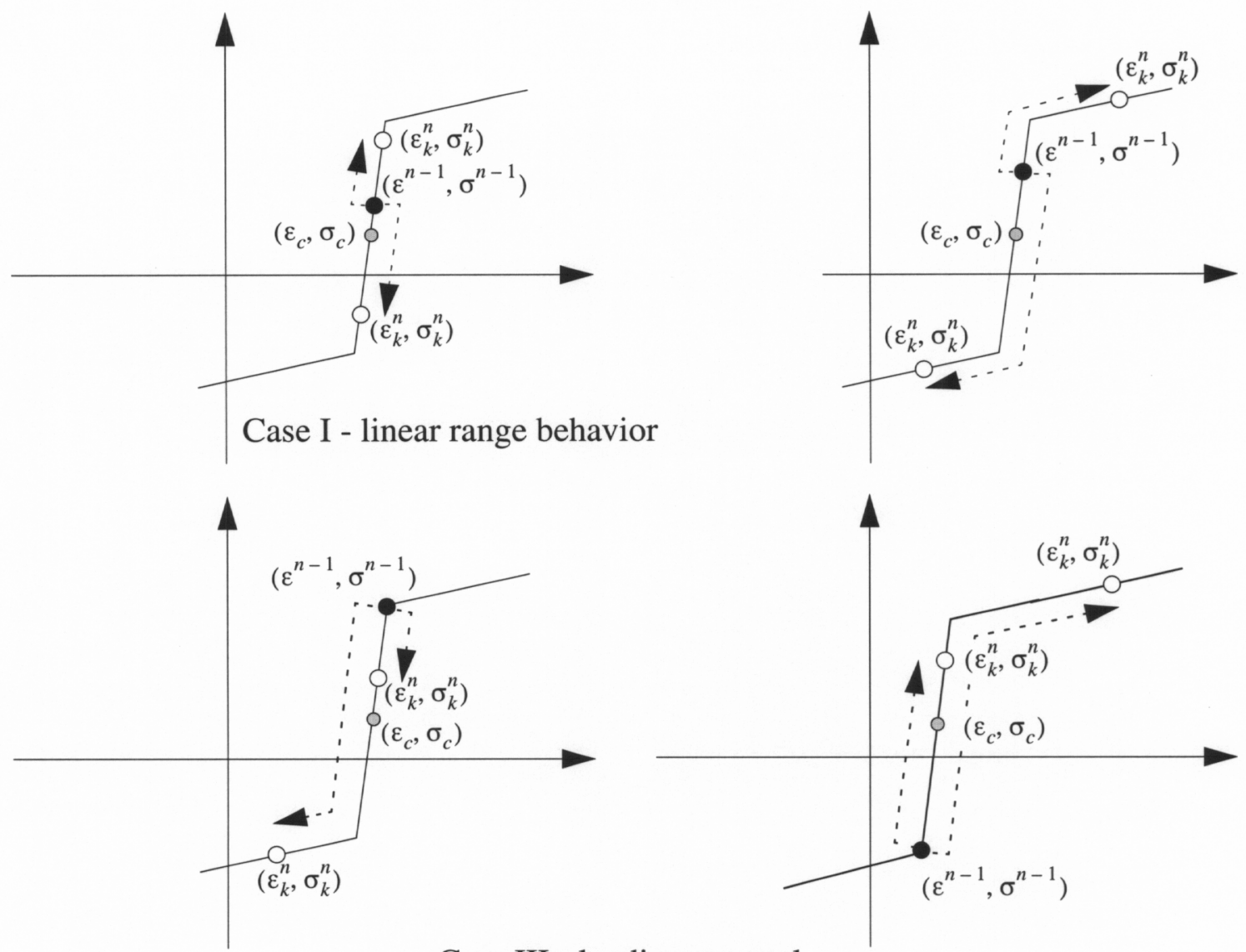

Case III - loading reversal

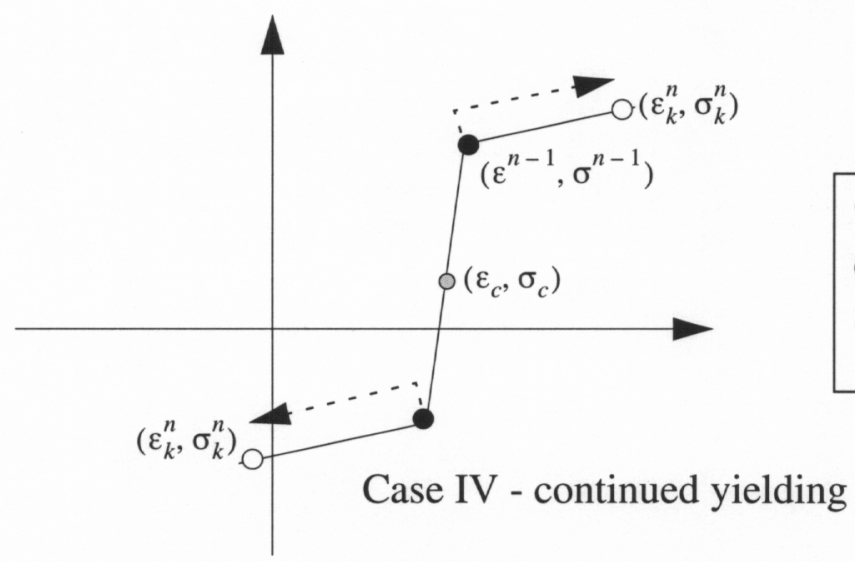

○ Center of yield region $\left(\varepsilon_{c}, \sigma_{c}\right)$

- Last fully converged state $\left(\varepsilon^{n-1}, \sigma^{n-1}\right)$

$\underset{\text { configuration }}{\text { Current equilibrium }}\left(\varepsilon_{k}^{n}, \sigma_{k}^{n}\right)$

FIGURE 31. Cases for consideration in stress determination

or

Defining

$$
\Delta \sigma=E_{y} \Delta \varepsilon+\left(E-E_{y}\right)\left(\varepsilon_{c}+\lambda-\varepsilon^{n-1}\right)
$$

$$
\Delta \varepsilon_{y} \equiv \varepsilon_{c}+\lambda-\varepsilon^{n-1}
$$




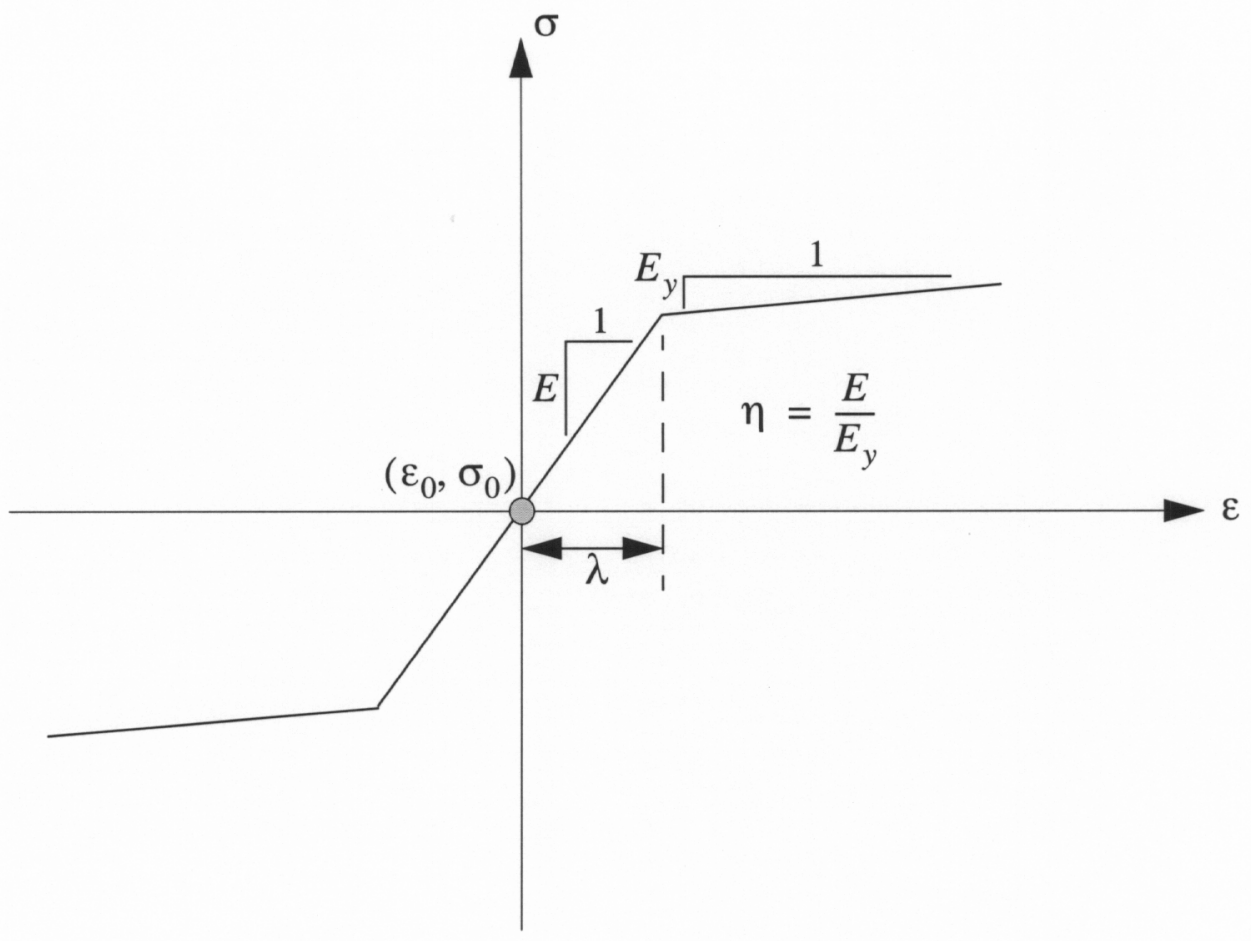

FIGURE 32. Stress-strain curve and parameters $\lambda$ and $\eta$.

which as shown in Figure 33 represents the strain increment from the last fully converged point to the yield point, the stress increment given in EQ. 249 can be written,

$$
\Delta \sigma=E_{y} \Delta \varepsilon+\left(E-E_{y}\right) \Delta \varepsilon_{y}
$$

or

$$
\Delta \sigma=E_{y} \Delta \varepsilon+\left(\eta E_{y}-E_{y}\right) \Delta \varepsilon_{y}
$$

EQ. 252 then reduces to,

$$
\Delta \sigma=E_{y}\left(\Delta \varepsilon+(\eta-1) \Delta \varepsilon_{y}\right)
$$

Defining the additional parameter,

$$
\rho \equiv \frac{\Delta \varepsilon_{y}}{\Delta \varepsilon}
$$

The stress increment of EQ. 253 can finally be written,

$$
\Delta \sigma=E_{y}[1+\rho(\eta-1)] \Delta \varepsilon
$$

For a negative strain increment, e.g. moving from point 1 to point 3 in Figure 33, a similar expression can be developed and it is found that EQ. 255 still holds if the appropriate strain to yield expression is employed, i.e.

$$
\Delta \varepsilon_{y}=\varepsilon_{c}-\lambda-\varepsilon^{n-1}
$$

With the appropriate definition of the strain to yield term as given in EQ. 250 and EQ. 256, the stress increment given in EQ. 255 is applicable for Case II in Figure 31 and in Case III when the current equilibrium configuration results in reverse direction yielding. 


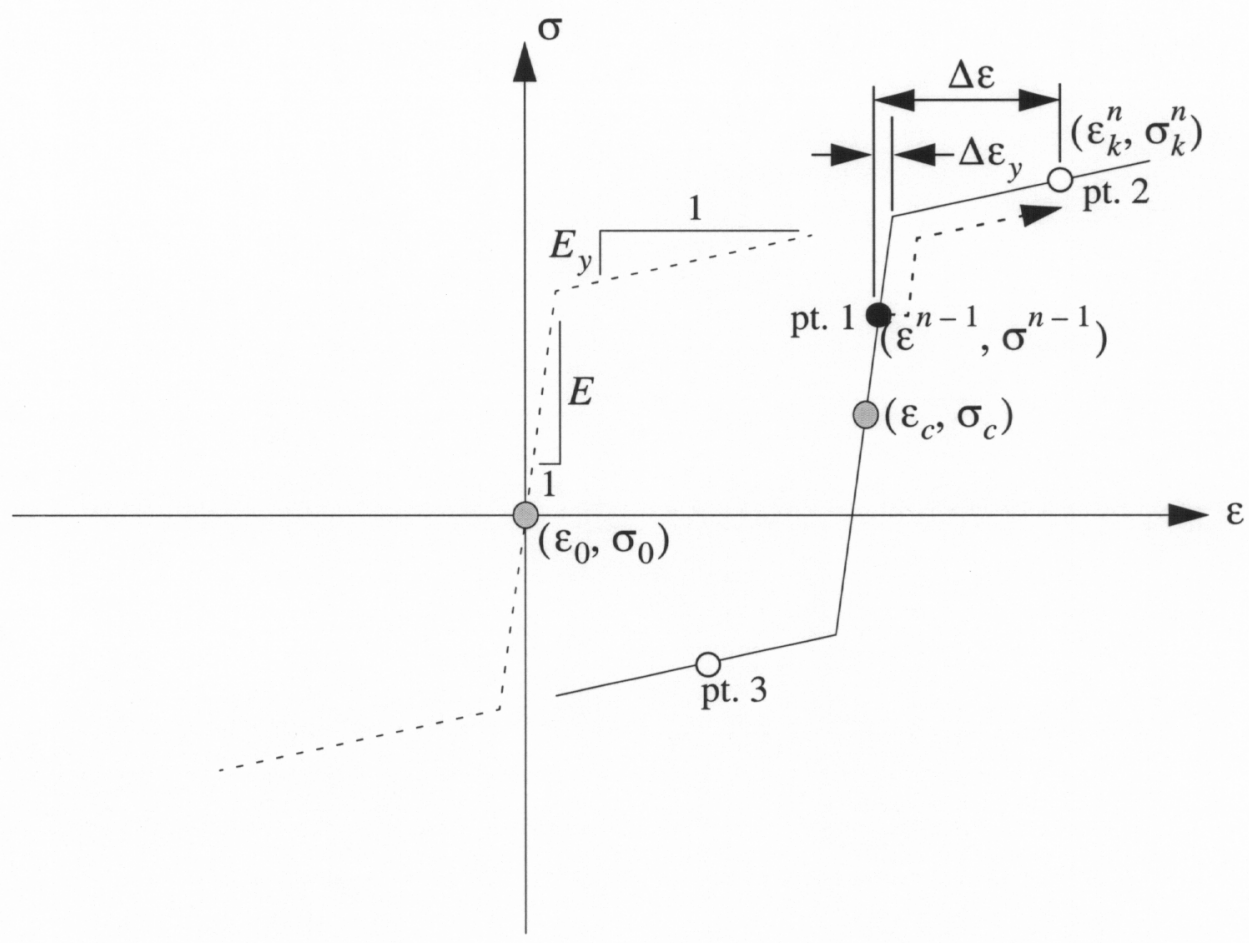

FIGURE 33. Integration of stress from the last fully converged state to the new current equilibrium configuration

In a Newton-Raphson incremental-iterative scheme, the stresses must be updated in a state determination for the current equilibrium configuration and the instantaneous stiffness of the structural system must be formed. For the state determination the total stress is found by obtaining the stress increment from EQ. 255 and adding the stress increment to the total stress at the last fully converged state. The instantaneous stiffness is found from the current rate of change of stress with respect to strain $\frac{d \sigma}{d \varepsilon}$. For the simple elasto-plastic model, the logic for accomplishing this, which will adequately capture all of the cases shown in Figure 31, is indicated in Figure 34.

When applied to the bilinear elasto-plastic model, the Newton-Raphson solution algorithm can experience serious divergence problems. In the well known paper by Matthies and Strang(Matthies and Strang) for example, the authors discuss some simple pathological cases in which the classical Newton-Raphson procedure diverges rapidly. A general observation, based on the author's numerical experimentation, is that since the Newton-Raphson procedure is a second order method, if the iterations do converge, converge is quite rapid. However, divergence is a distinct possibility unless special controls are implemented. In the current work, a special control was implemented to assist in convergence of the Newton-Raphson procedure at the global finite element level. The procedure which was implemented consists of using an approximation of the true tangent stiffness when 


\section{Define yield indicator variables}

yindint $\equiv$ yield indicator for stress integration

yindstf $\equiv$ yield indicator for stiffness

\section{Perform preloadstep loop initialization}

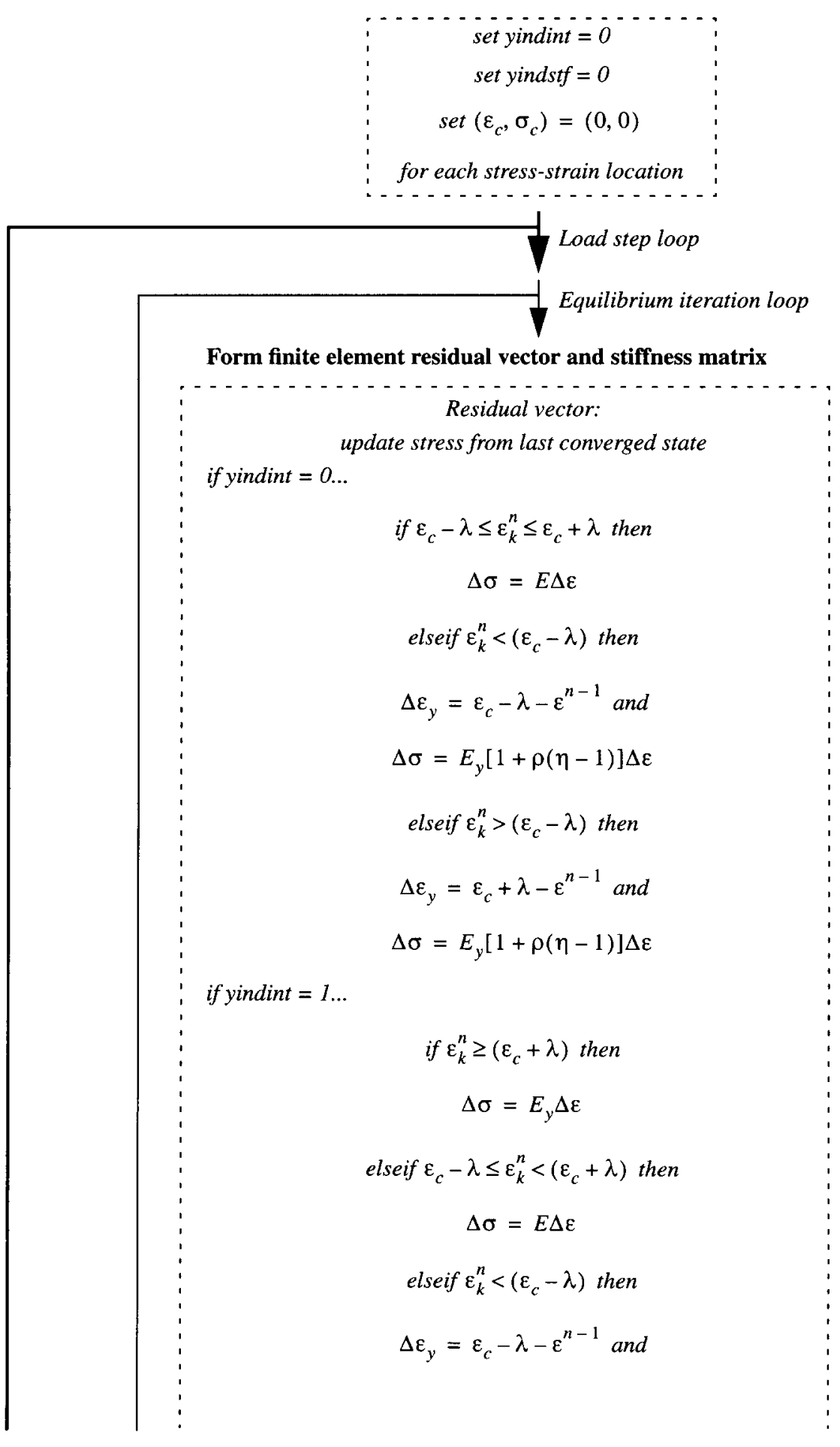




$$
\Delta \sigma=E_{y}[1+\rho(\eta-1)] \Delta \varepsilon
$$

if yindint $=-1 \ldots$

$$
\begin{gathered}
\text { if } \varepsilon_{k}^{n} \leq\left(\varepsilon_{c}-\lambda\right) \text { then } \\
\Delta \sigma=E_{y} \Delta \varepsilon \\
\text { elseif }\left(\varepsilon_{c}-\lambda\right)<\varepsilon_{k}^{n} \leq \varepsilon_{c}+\lambda \text { then } \\
\Delta \sigma=E \Delta \varepsilon \\
\text { elseif } \varepsilon_{k}^{n} \geq\left(\varepsilon_{c}+\lambda\right) \text { then } \\
\Delta \varepsilon_{y}=\varepsilon_{c}+\lambda-\varepsilon^{n-1} \text { and } \\
\Delta \sigma=E_{y}[1+\rho(\eta-1)] \Delta \varepsilon \\
\hdashline:=:=:=:=: \\
\text { Stiffness matrix: }
\end{gathered}
$$

form instantaneous stiffness matrix for the current equilibrium configuration

if $\left(\varepsilon_{c}-\lambda\right)<\varepsilon_{k}^{n}<\left(\varepsilon_{c}+\lambda\right)$ then yindst $=0$

if $\varepsilon_{k}^{n} \leq \varepsilon_{c}-\lambda$ or $\varepsilon_{k}^{n} \geq\left(\varepsilon_{c}+\lambda\right)$ then yindstf $=1$ if yindstf $=0$...

$$
\frac{d \sigma}{d \varepsilon}=E
$$

if yindstf $=1 \ldots$

$$
\frac{d \sigma}{d \varepsilon}=E_{y}
$$

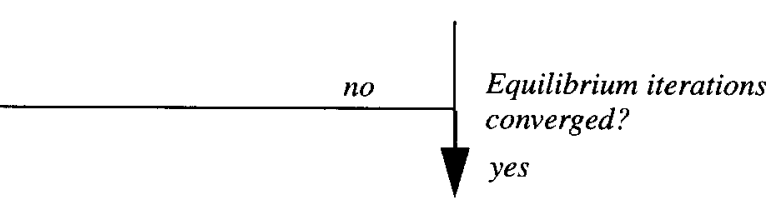

\section{Update yield region and yield indicators}

$$
\begin{gathered}
\text { after convergence }\left(\varepsilon_{k}^{n}, \sigma_{k}^{n}\right) \rightarrow\left(\varepsilon_{k+1}, \sigma_{k+1}\right) \\
\varepsilon_{t o p}=\varepsilon_{c}+\lambda \\
\varepsilon_{b o t t o m}=\varepsilon_{c}-\lambda \\
\text { if } \varepsilon_{k+1} \geq \varepsilon_{t o p} \text { then } \\
y i n d i n t=1 \\
\sigma_{\text {trans }}=\sigma_{k+1}-\left(\sigma_{c}+\sigma_{y}\right) \\
\sigma_{c}=\sigma_{c}+\sigma_{\text {trans }} \\
\varepsilon_{c}=\varepsilon_{k+1}-\lambda
\end{gathered}
$$




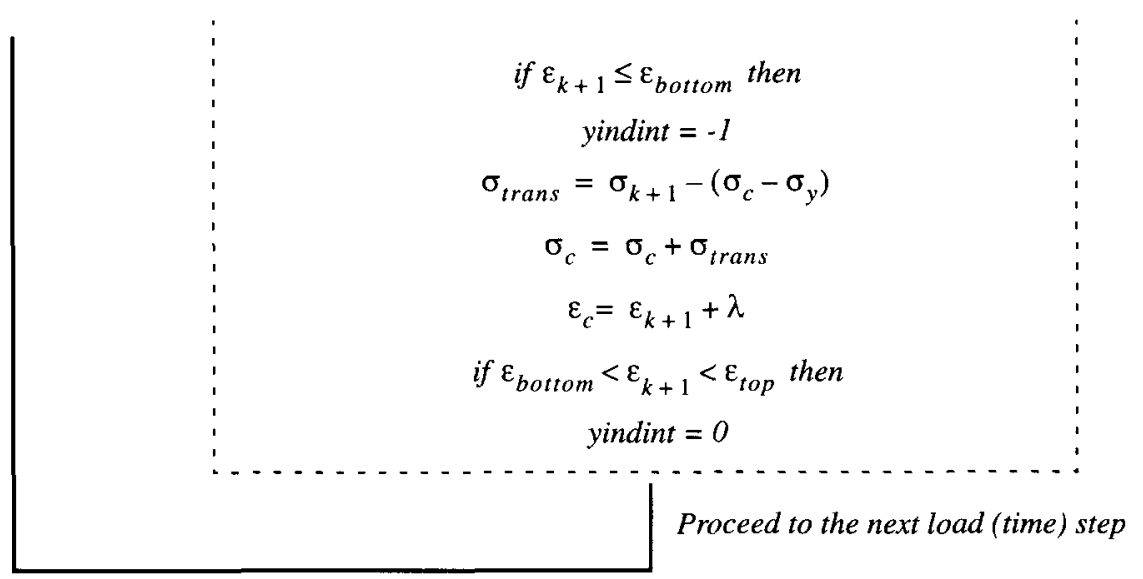

FIGURE 34. Flowchart for implementation of elasto-plastic model in a Newton-Raphson solution framework

yielding occurs. Thus instead of using $\frac{d \sigma}{d \varepsilon}=E_{y}$ to characterize the instantaneous stiffness, an approximation is used, i.e.

$$
\frac{d \sigma}{d \varepsilon} \Rightarrow \frac{E_{y}+\tau E}{2}
$$

Utilization of the approximate expression in EQ. 257 significantly improved the convergence of the Newton-Raphson scheme at the global finite element level. This approximation does lead to higher compute costs as a result of an increased number of equilibrium iterations. Since the true instantaneous stiffness is not being utilized, convergence is no longer second order. Based on numerical experimentation, appropriate values of $\tau$, which enhance the stability of the Newton-Rapson procedure, with a minimal impact on convergence rate, are in the range of 0.5 to 0.7 .

\subsection{References}

Newmark, N.M., A method of computation for structural dynamics, ASCE Journal of Engineering Mechanics Division, 85, 1959.

Przemieniecki, J.R., Theory of Matrix Structural Analysis, McGraw-Hill, 1968.

Hildebrand, F.B., Introduction to Numerical Analysis, McGraw-Hill, 1956.

Matthies, H. and G. Strang, The solution of nonlinear finite element equations, International Journal for Numerical Methods in Engineering, 14, 1613-1626, 1979. 


\subsection{Acknowledgement}

This reserach was funded by the Laboratory Directed Research and Development Program at the Lawrence Livermore National Laboratory. The authors wish to express their sincere appreciation for the continued support and encouragement of Dr. Rokaya Al-Ayat and Dr. Karl Van Bibber of the Laboratory Science and Technology Office.

This work was performed at the Lawrence Livermore National Laboratory under the auspices of the United States Department of Energy, contract W-7405-Eng-48. 


\section{APPENDIX 1}

REGIONAL GROUND MOTION SIMULATION EXAMPLE 


\subsection{E3D Wave Propagation Example}

An E3D wave propagation simulation for the NEVADA environment is presented in this Appendix. In this particular example, the objective is to simulate the 1989 Barnwell event at the Nevada Test Site and to compute the seismic wavefield in southern Nevada and the strong ground motions within the Las Vegas Basin [Larsen, 2002]. The synthetic data can be compared to observed data as a means of validating the model.

The area of interest is shown in Figure 35. The horizontal extent of the finite-difference grid is indicated by the blue rectangle. It is $300 \mathrm{~km}$ long and $200 \mathrm{~km}$ wide. The vertical extent is $100 \mathrm{~km}$. Seismic energy is propagated from the Barnwell event (red star) at the Nevada test site (yellow outline) to the Las Vegas Basin (flat area within the white box).

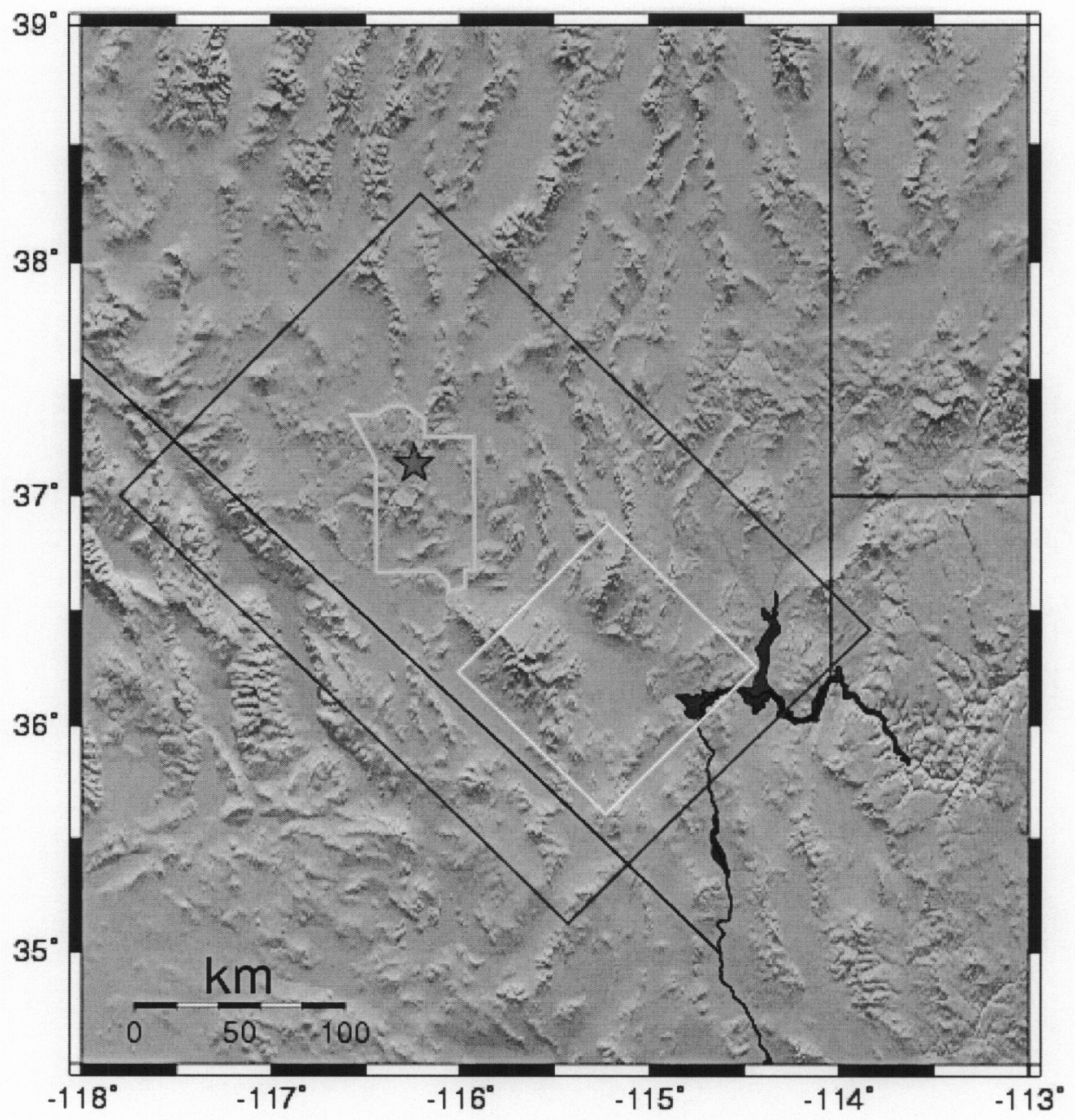

FIGURE 35. Southern Nevada simulation region (blue box) includes the Nevada Test Site (yellow outline) and the Las Vegas Basin (white box). Barnwell test location is indicated by red star. 

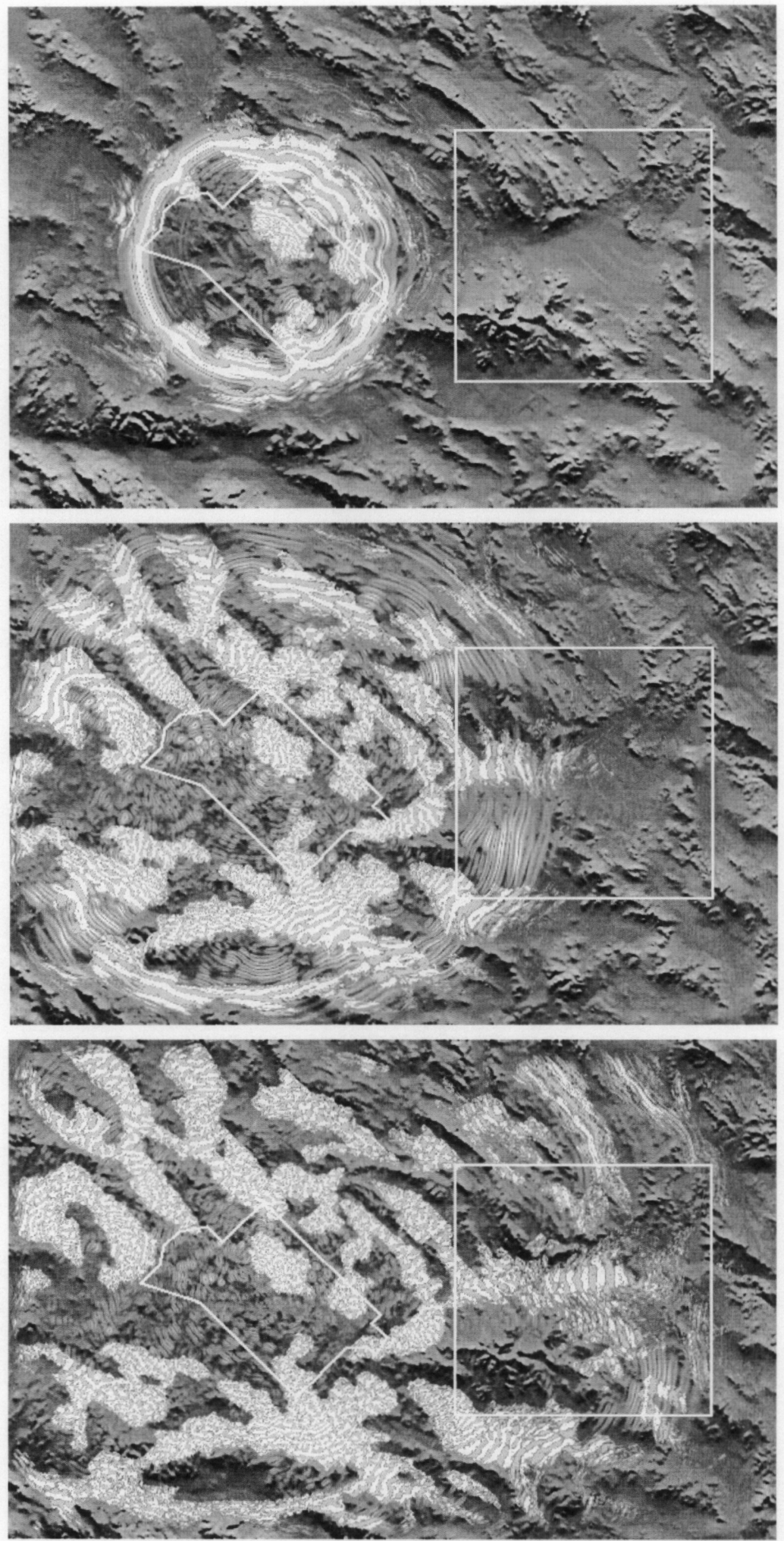

FIGURE 36. Seismic wavefield (snapshots) at successive times following Barnwell event. 
In this example, the numerical spacing of the finite-difference grid is $0.125 \mathrm{~km}$. The actual yield of the Barnwell event is not available, for this example simulation the Barnwell event was simply idealized as a $150 \mathrm{KT}$ explosion ${ }^{1}$, which is equivalent to a magnitude 5.7 earthquake. The simulated source is located at a depth of $500 \mathrm{~m}$, which is comparable to the actual source depth of $600 \mathrm{~m}$. A Gaussian pulse with an upper frequency content of about $1.0 \mathrm{hz}$ is used as the source time function.

The geologic model is based on Model Assembler 2.1, a product of the University of Nevada at Reno [Concha-Dimas et al., 2002]. In particular, this model uses a depth-tobasement profile to characterize the $5 \mathrm{~km}$ deep Las Vegas Basin. This depth-to-basement profile is based on gravity and other geophysical information [Langenheim et al., 2001]. The velocity structure of the smaller basins in this region is obtained using structural rules from Model Assembler 2.1. A 1-D regional velocity model is used for depths greater than $5 \mathrm{~km}$. This particular example simulation does not include topography and it does not include attenuation.

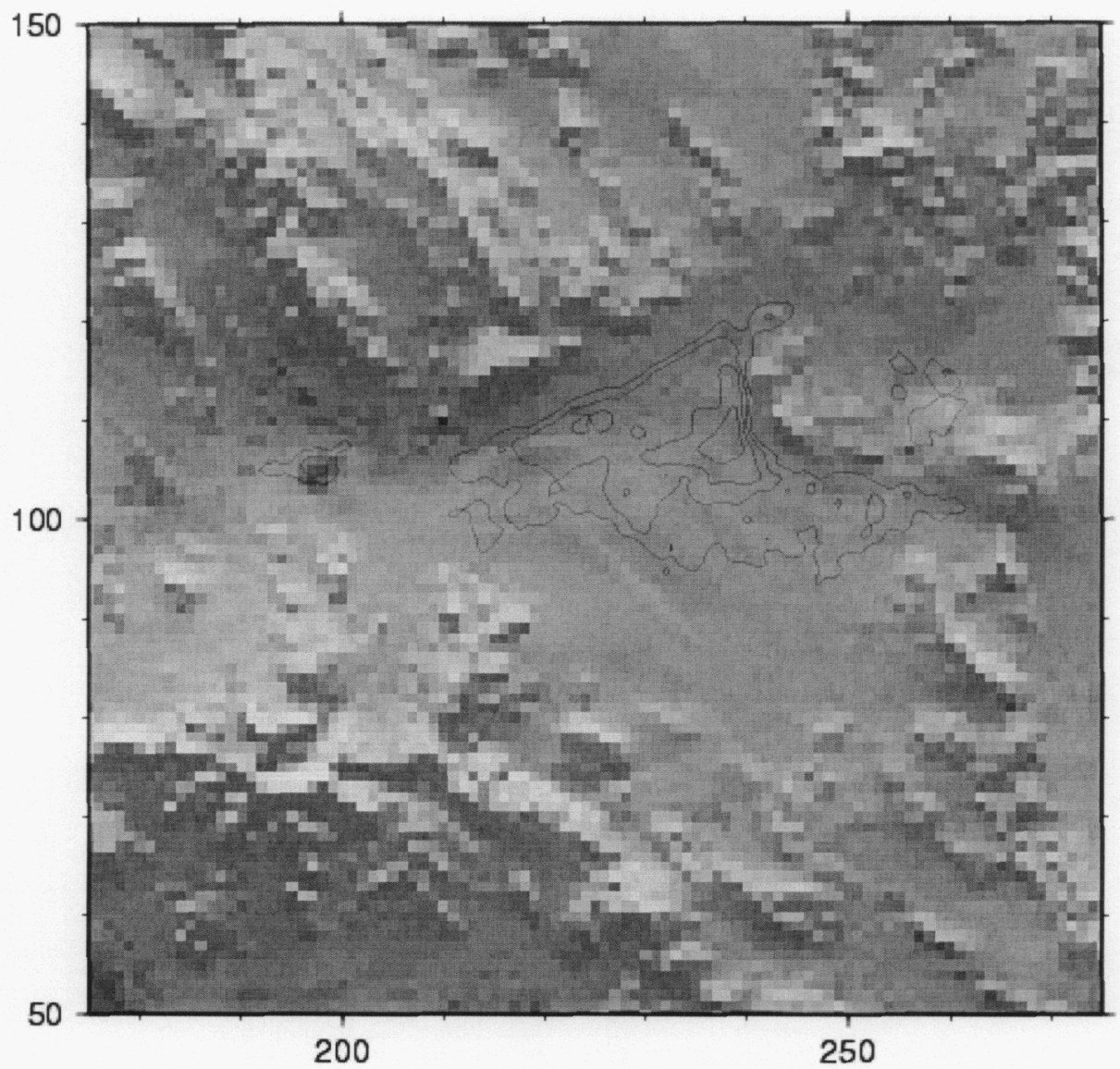

FIGURE 37. Maximum ground motions in the Las Vegas Basin. Green are ground velocities between 0.04 and $0.08 \mathrm{~cm} / \mathrm{s}$. Red are ground velocities above $0.08 \mathrm{~cm} / \mathrm{s}$.

1. Publically available information on the Barnwell event lists the yield as 20-150KT range. 
The seismic wavefield (snapshots) at three times during the simulation is given in Figure 36. Shown are the $\mathrm{P}$ and $\mathrm{S}$ velocity potentials. The color indicates the type of energy, with shear energy represented by green and compressional energy represented by red. Most of the energy is shear. The intensity of the color indicates the amplitude of the seismic motion (in essence, the velocity of the ground motion). The affect of basin propagation is obvious. The coherence of the seismic wavefield is significantly disrupted as seismic energy is trapped and reverberated within the basins.
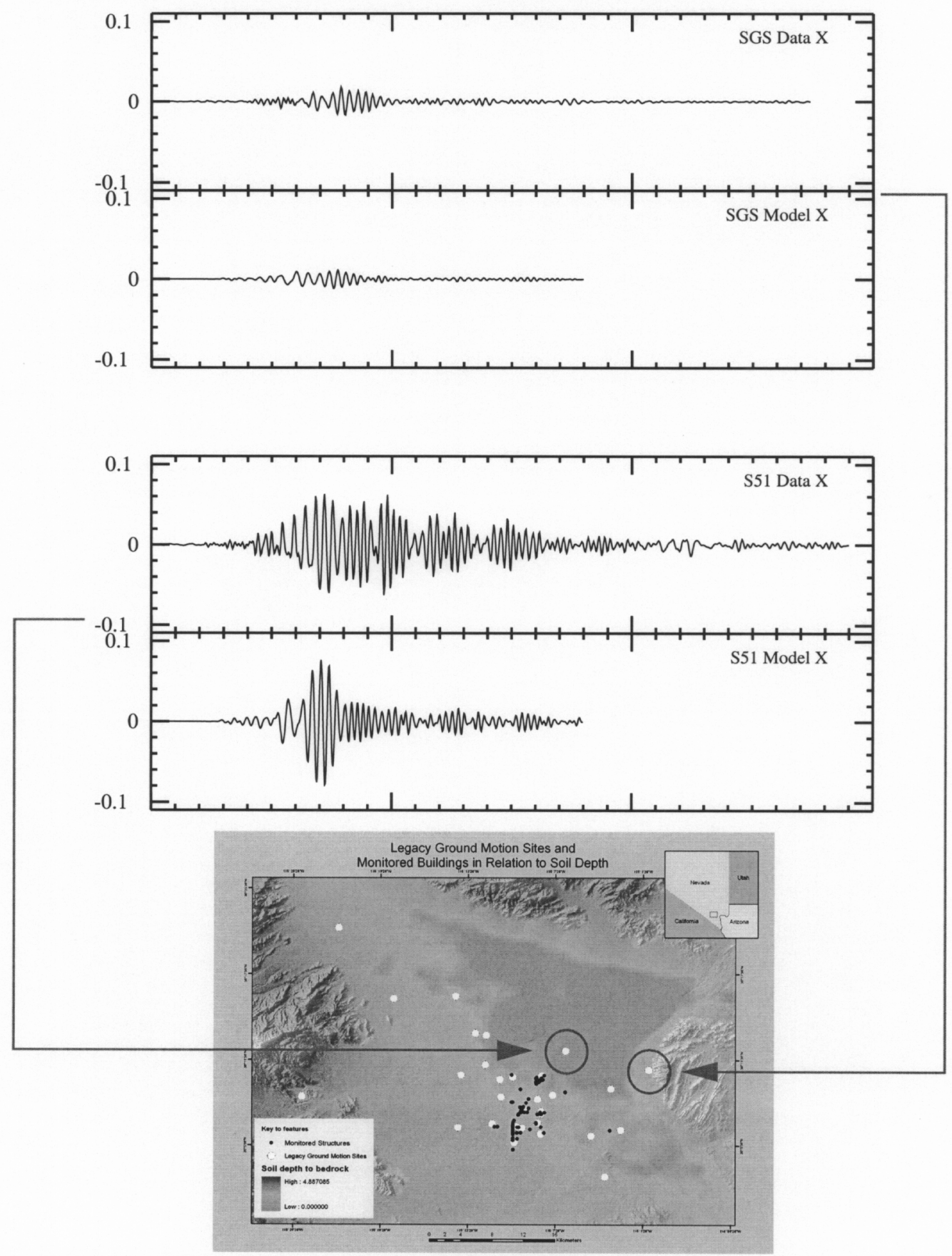

FIGURE 38. Comparison between data and simulation for soil (S51) and rock (SGS) sites within and on the peripheral of the Las Vegas Basin. Shown is the X (southeast) component of ground velocity. The amplitude scale is in $\mathrm{cm} / \mathrm{s}$. The horizontal axis goes to $300 \mathrm{~s}$. 
The maximum ground motions within the Las Vegas Basin are shown in Figure 37. Areas of green indicate maximum ground velocities between 0.04 and $0.08 \mathrm{~cm} / \mathrm{s}$. Areas of red indicate maximum ground velocities above $0.08 \mathrm{~cm} / \mathrm{s}$. The depth-to-basement within the Las Vegas Basin is shown by the $1 \mathrm{~km}$ contour intervals (maximum basin depth is about 5 $\mathrm{km})$. In general, maximum motions are observed within the basin. There is some correlation between amplitude of motion and basin depth, although strong shaking is also indicated in shallower parts of the basin. In part, amplitudes in the northwest (left) portion of the basin may be larger because this region is closer to the source.

A comparison between observed and simulated data at soil (S51) and rock (SGS) sites within and on the peripheral of the Las Vegas Basin is given in Figure 38. Shown is the $\mathrm{X}$ (southeast) component of ground velocity $(\mathrm{cm} / \mathrm{s})$. The maximum amplitudes agree very well. In addition, there is good agreement in the general character of the signal although differences are also observed (especially at S51, the soil site). These differences could be due to unmodeled complexities in the geology, topography, or some other effect. Comparisons at other sites tend to show similar if not between agreement. The positive comparison between model and observation suggests that ground motions in areas of the Las Vegas Basin that have no historical observations can be estimated by simulation.

\subsection{References}

Concha-Dimas, T. Rasmussen, J. N. Louie, S. Smith, W. Thelen, A. Pancha, J. G. Anderson, Las Vegas Basin seismic response project: developing a community velocity model for NTS and Las Vegas, AGU Fall Meeting, 83, 2002.

Langenheim, V., J. Grow, R. Jachens, G. Dixon, and J. Miller, Geophysical constraints on the location and geometry of the Las Vegas Valley Shear Zone, Nevada, Tectonophysics, 20, 189-209, 2001.

Larsen, S., Las Vegas Basin seismic response project: 3-D finite-difference ground motion simulations, AGU Fall Meeting, 83, 2002. 


\section{APPENDIX 2}

STORAGE AND OPERATIONS FOR SYMMETRIC, BANDED MATRIX IN COMPACTED SKYLINE FORM 


\subsection{Vector operations}

The solution of simultaneous equations and the solution of the free vibration eigenproblems in the NEVADA program is based on a skyline form of storage of the model system matrix. The equation solutions and storage format follow that of Bathe and Wilson (Bathe and Wilson, ) and the Fortran subroutines COLSOL and SSPACE provided in the referenced book have been implemented in NEVADA. The compacted skyline storage saves only the terms below the skyline of the symmetric matrix as indicated in Figure 39. The time history solution of the incremental equations of motion requires computation of matrix-vector products. The Fortran logic and syntax for carrying out a matrix-vector multiplication is indicated below

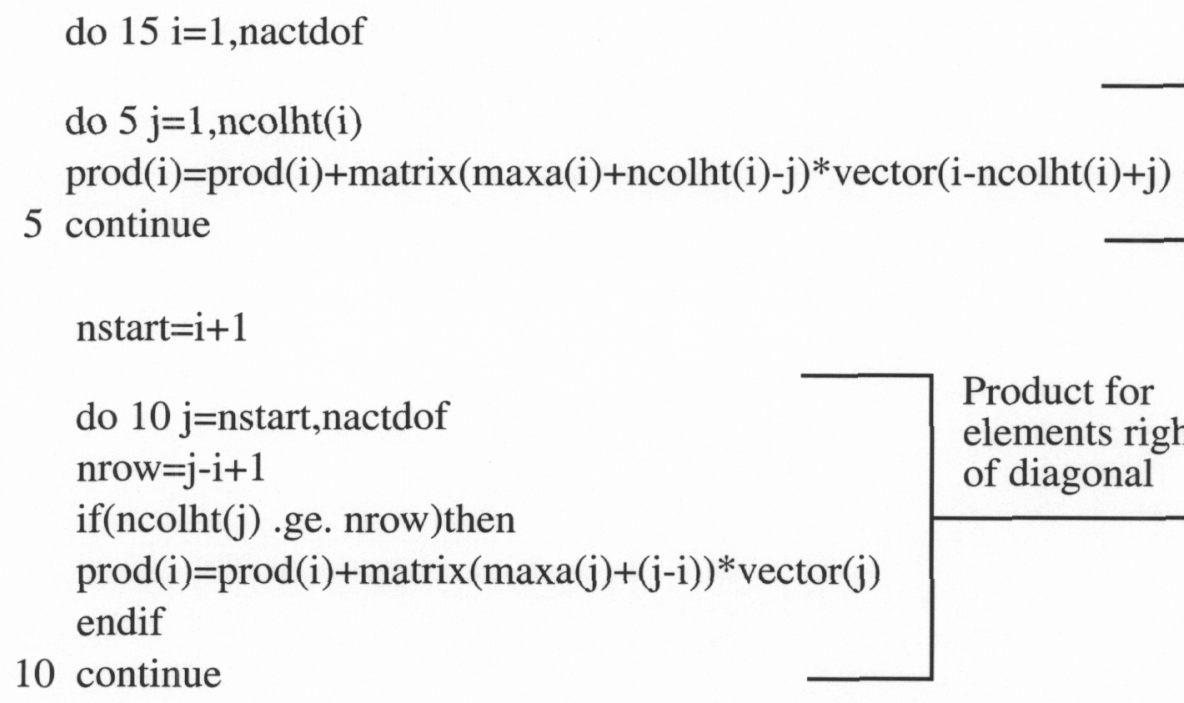

15 continue

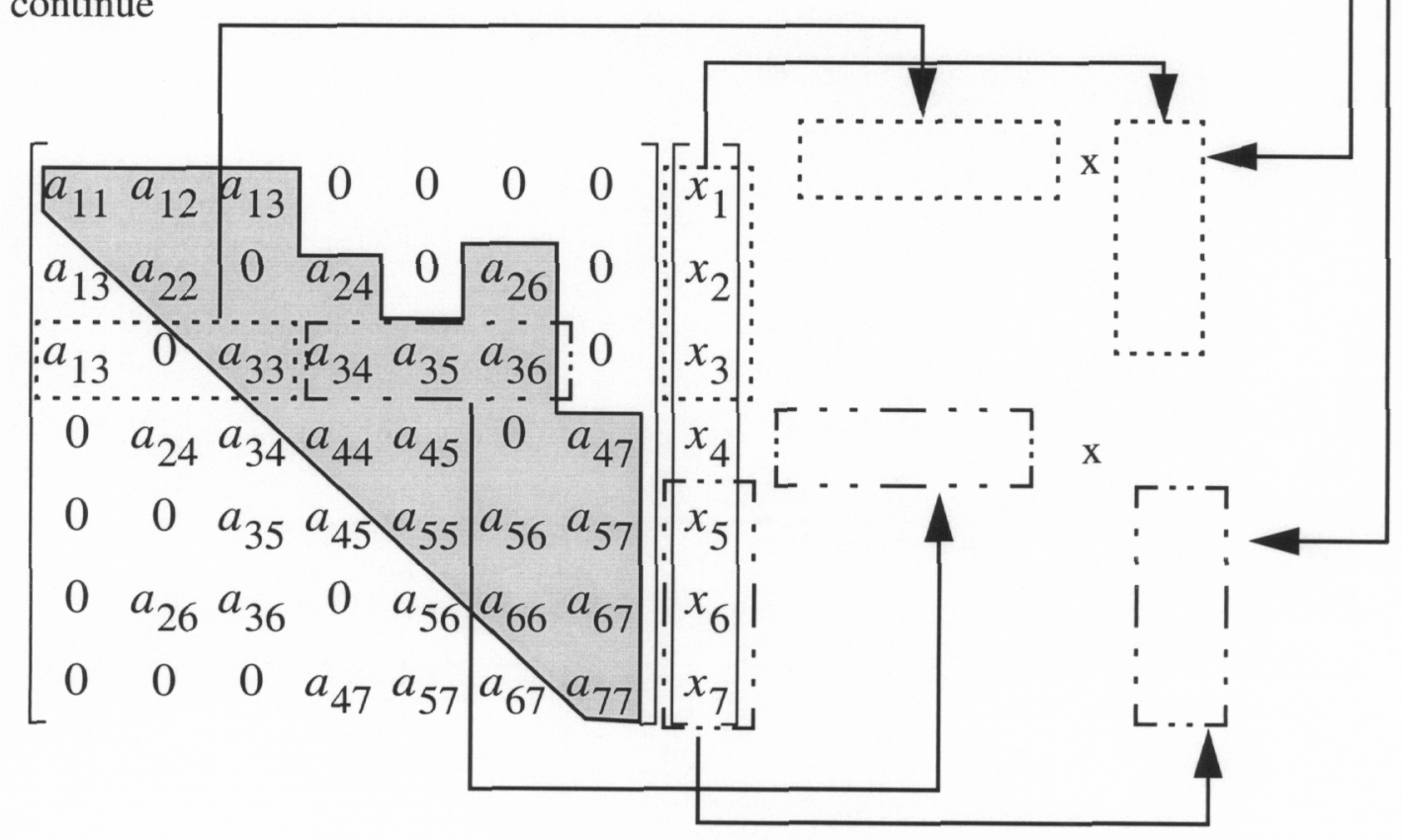




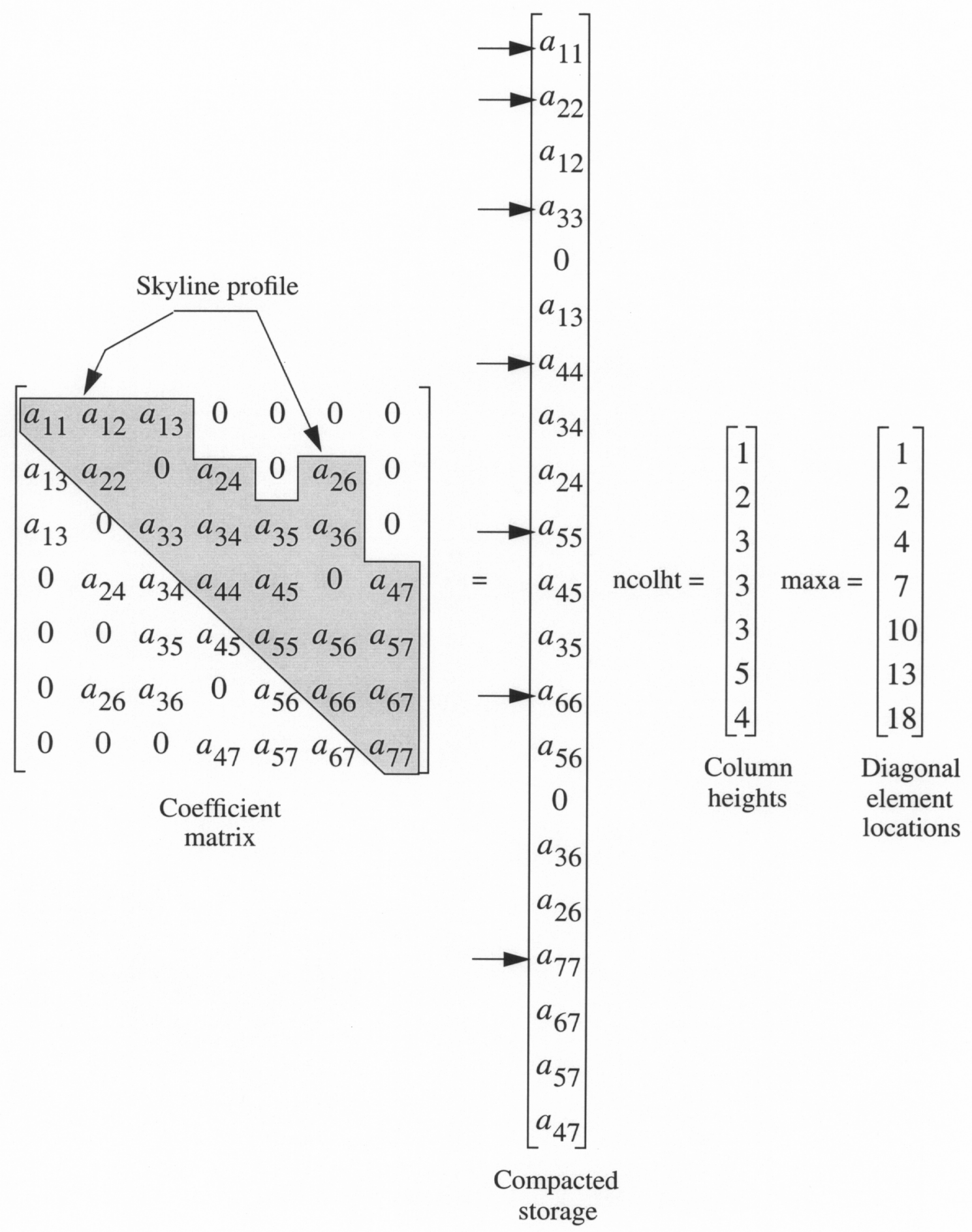

FIGURE 39. Compacted skyline storage of a symmetric banded coefficient matrix. 


\subsection{References}

Bathe, K.J. and E.L. Wilson, Numerical Methods in Finite Element Analysis, Precentice Hall, 1976. 


\begin{abstract}
APPENDIX 3
REPRESENTATIVE STEEL MOMENT RESISTING

FRAME BUILDINGS AND VERIFICATION PROBLEMS
\end{abstract}




\subsection{Representative Steel Frame Structures}

To evaluate the performance of the NEVADA building model, four typically designed representative steel moment frame buildings for UBC zone 3 were created. These models were carefully designed in collaboration with Professor Hassan Astaneh of UC Berkeley to ensure realistic dimensions, beam and column section properties and mass values. The three, nine and twenty story buildings were similar to representative models developed as part of the SAC steel structure project ${ }^{1}$. The forty story frame building was also representative of a UBC zone 3 structure and an existing San Francisco high-rise structure was used as a starting point and modified to meet zone 3 design criteria. The dimensions of the building structures at identical scale are shown in Figure 40.
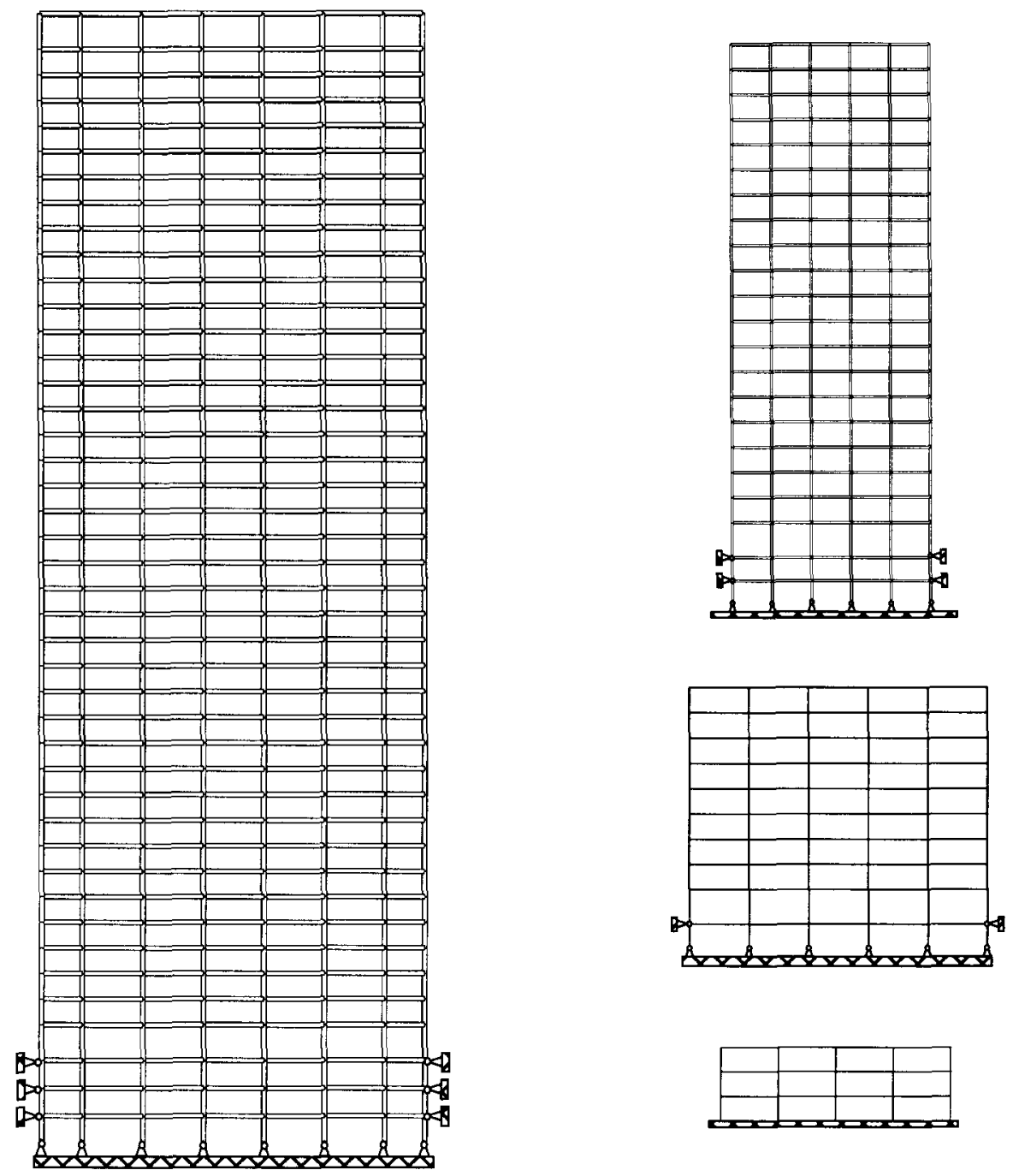

FIGURE 40. Representative forty, twenty, nine and three story steel moment resisting frames.

1. The SAC project was funded by the Federal Emergency Management Agency to solve the problem of brittle behavior of welded steel frame structures that surfaced in the January 17, 1994 Northridge California Earthquake. 
The properties of the four frames are summarized in Table 2

TABLE 2. Properties of representative buildings

\begin{tabular}{|c|c|c|c|c|}
\hline $\begin{array}{c}\text { Steel } \\
\text { building } \\
\text { height }^{\mathrm{a}}\end{array}$ & $\begin{array}{c}\mathrm{M}_{1} \\
\text { Effective total mass in } \\
\text { frame for lateral } \\
\text { loading }\end{array}$ & $\begin{array}{c}\mathbf{M}_{2}, \mathbf{P}_{\Delta} \\
\text { Effective total vertical } \\
\text { mass in frame for } \\
\text { gravity initialization } \\
\text { and } \mathbf{P}-\Delta \text { loads from } \\
\text { internal frames }\end{array}$ & $\begin{array}{c}\text { Frequencies } \\
\text { (periods) of } \\
\text { planar frame } \\
\text { - no gravity } \\
\text { initialization }\end{array}$ & $\begin{array}{c}\text { Frequencies } \\
\text { (periods) of } \\
\text { planar frame } \\
\text { - including gravity } \\
\text { initialization }\end{array}$ \\
\hline 3 story & $\begin{aligned} \mathrm{M}_{1}= & 8.623 \times 10^{5} \mathrm{~N}-\mathrm{s}^{2} / \mathrm{m} \\
& \left(1.901 \times 10^{6} \mathrm{lb}\right)\end{aligned}$ & $\begin{array}{c}\mathrm{M}_{2}=3.531 \times 10^{5} \mathrm{~N}-\mathrm{s}^{2} / \mathrm{m} \\
\left(7.785 \times 10^{5} \mathrm{lb}\right) \\
\mathrm{P}_{\Delta}=1.47 \times 10^{7} \mathrm{~N} \\
\left(3.306 \times 10^{6} \mathrm{lb}\right)\end{array}$ & $\begin{array}{l}1.10 \mathrm{~Hz}(0.909 \mathrm{sec}) \\
3.49 \mathrm{~Hz}(0.287 \mathrm{sec}) \\
5.96 \mathrm{~Hz}(0.168 \mathrm{sec})\end{array}$ & \\
\hline 9 story & $\begin{aligned} \mathrm{M}_{1}= & 2.992 \times 10^{6} \mathrm{~N}-\mathrm{s}^{2} / \mathrm{m} \\
& \left(6.595 \times 10^{6} \mathrm{lb}\right)\end{aligned}$ & 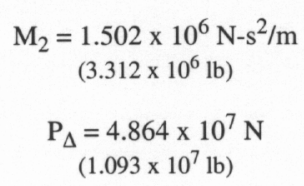 & $\begin{array}{l}0.486 \mathrm{~Hz}(2.06 \mathrm{sec}) \\
1.26 \mathrm{~Hz}(0.794 \mathrm{sec}) \\
2.28 \mathrm{~Hz}(0.439 \mathrm{sec})\end{array}$ & $\begin{array}{l}0.480 \mathrm{~Hz}(2.08 \mathrm{sec}) \\
1.25 \mathrm{~Hz}(0.800 \mathrm{sec}) \\
2.27 \mathrm{~Hz}(0.441 \mathrm{sec})\end{array}$ \\
\hline 20 story & $\begin{aligned} \mathrm{M}_{1}= & 3.867 \times 10^{6} \mathrm{~N}-\mathrm{s}^{2} / \mathrm{m} \\
& \left(8.525 \times 10^{6} \mathrm{lb}\right)\end{aligned}$ & $\begin{array}{c}\mathrm{M}_{2}=2.159 \times 10^{6} \mathrm{~N}-\mathrm{s}^{2} / \mathrm{m} \\
\left(4.760 \times 10^{6} \mathrm{lb}\right) \\
\mathrm{P}_{\Delta}=5.224 \times 10^{7} \mathrm{~N} \\
\left(1.174 \times 10^{7} \mathrm{lb}\right)\end{array}$ & $\begin{array}{l}0.375 \mathrm{~Hz}(2.67 \mathrm{sec}) \\
0.960 \mathrm{~Hz}(1.04 \mathrm{sec}) \\
1.62 \mathrm{~Hz}(0.617 \mathrm{sec})\end{array}$ & $\begin{array}{l}0.362 \mathrm{~Hz}(2.76 \mathrm{sec}) \\
0.934 \mathrm{~Hz}(1.07 \mathrm{sec}) \\
1.58 \mathrm{~Hz}(0.633 \mathrm{sec})\end{array}$ \\
\hline 40 story & $\begin{aligned} \mathrm{M}_{1}= & 1.067 \times 10^{7} \mathrm{~N}-\mathrm{s}^{2} / \mathrm{m} \\
& \left(2.354 \times 10^{7} \mathrm{lb}\right)\end{aligned}$ & $\begin{array}{c}\mathrm{M}_{2}=8.982 \times 10^{6} \mathrm{~N}-\mathrm{s}^{2} / \mathrm{m} \\
\left(1.980 \times 10^{7} \mathrm{lb}\right) \\
\mathrm{P}_{\Delta}=2.037 \times 10^{8} \mathrm{~N} \\
\left(4.578 \times 10^{7} \mathrm{lb}\right)\end{array}$ & $\begin{array}{l}0.189 \mathrm{~Hz}(5.29 \mathrm{sec}) \\
0.505 \mathrm{~Hz}(1.98 \mathrm{sec}) \\
0.792 \mathrm{~Hz}(1.26 \mathrm{sec})\end{array}$ & $\begin{array}{l}0.182 \mathrm{~Hz}(5.49 \mathrm{sec}) \\
0.491 \mathrm{~Hz}(2.04 \mathrm{sec}) \\
0.771 \mathrm{~Hz}(1.30 \mathrm{sec})\end{array}$ \\
\hline
\end{tabular}

a. Assuming rectangular building cross-section and all lateral load resistance provided by the four perimeter moment resisting frames (internal frames non-moment connections). Building designed per 1988 UBC seismic zone 3 requirements.

b. Effective mass for lateral seismic loading (including contributing mass from internal frames)

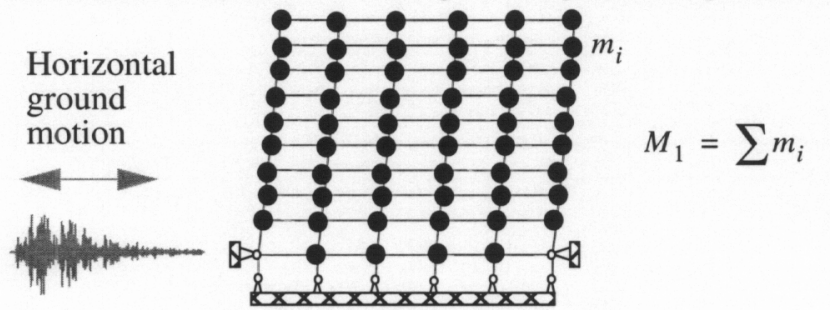

Mass of frame plus contributing mass of internal frames during lateral loading

c. Effective mass for gravity load initialization plus P- $\Delta$ loads from internal frames

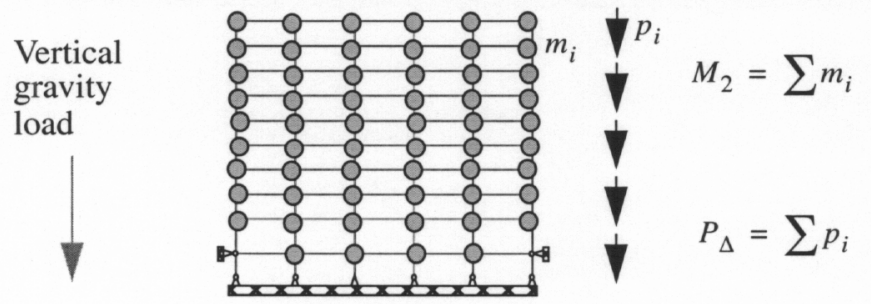

Effective mass contribution from frame vertical loads (DL+LL) plus vertical load contribution by orthogonal beams connected to frame

Effective P- $\Delta$ loads from side-sway of internal simply connected frames 
The section properties and computed natural frequencies of each of these buildings are shown in Figure 41 through Figure 45. For each structure the natural frequencies computed with the NEVADA framework are compared with the frequencies computed with the LLNL GEMINI linear elastic general purpose finite element program. The NEVADA and GEMINI models exhibit excellent agreement.

\subsection{Nonlinear analysis results}

A large suite of transient nonlinear analysis problems were computed to verify the nonlinear analysis capabilities of the NEVADA framework. A selected subset of illustrative problems are summarized here.

\subsection{Inelastic axial deformation of a steel beam}

A W14x176 wide flange beam was axially loaded with a concentric load that was applied slowly over three seconds. The amplitude of the load was adjusted so that the initial loading resulted in significant plastic deformation of the beam. at the end of three seconds, the applied load was abruptly removed and the beam was allowed to vibrate freely in the axial direction. The response of the beam, as computed with NEVADA and the LLNL general purpose nonlinear finite element program NIKE3D is shown in Figure 46. Both models employed spectral damping with mass and stiffness proportional damping. The two models exhibit excellent agreement.

\subsection{Inelastic lateral deformation of a steel beam}

The same wide flange beam was loaded in similar fashion only with a load in the lateral direction of the beam. This loading resulting in progressive yielding in the beam crosssection. At three seconds the tip load was suddenly released and the beam was allowed to vibrate freely. In this example the beam was undamped. The responses computed with NEVADA and NIKE3D models are indicated in Figure 47. For this example there was no damping incorporated in the models and thus the models vibrated freely without energy decay after the initial loading.

\subsection{Linear and nonlinear analyses of a planar building}

The nine story building shown in Figure 42 was subjected to near-field earthquake motions from the Landers California Earthquake. Linear and nonlinear transient solutions computed with NEVADA and NIKE3D models are shown in Figure 48. The models exhibit excellent agreement. The long period near-field displacement pulse from the ground motion record causes significant inelastic deformation of this building frame. The permanent lateral displacement at the roof of the building is on the order of 11 inches. 


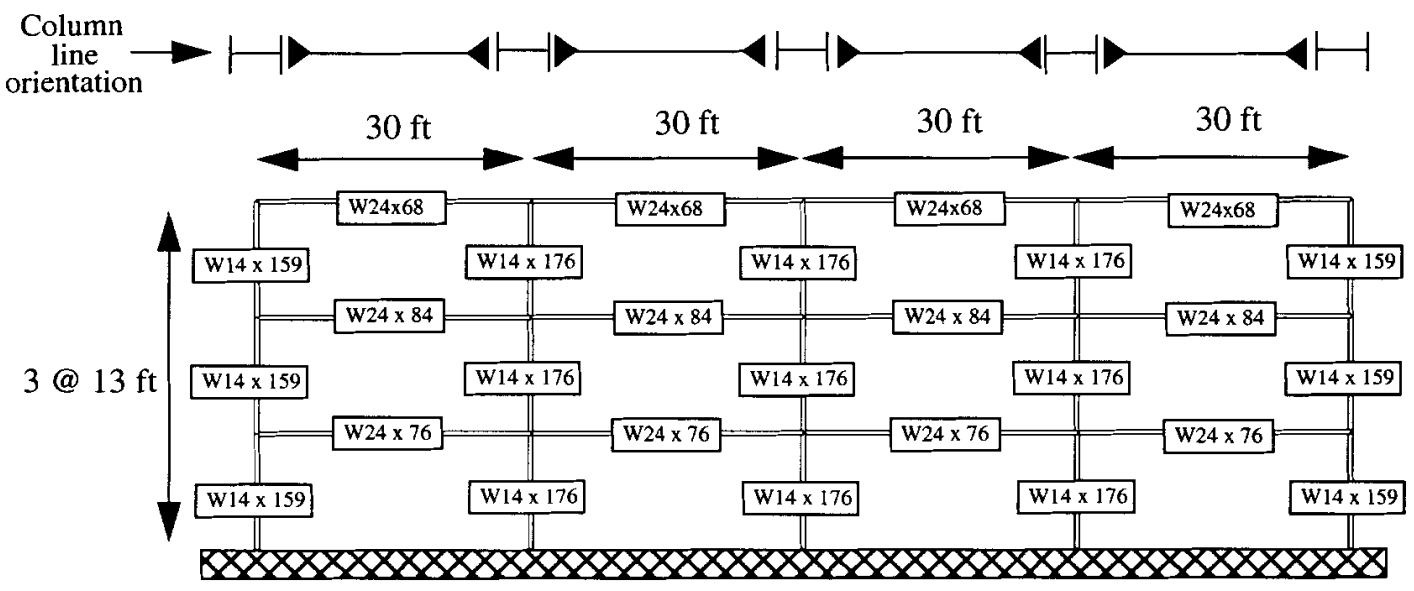

Total tributary weight to frame $=1.901 \times 10^{6} \mathrm{lb}$
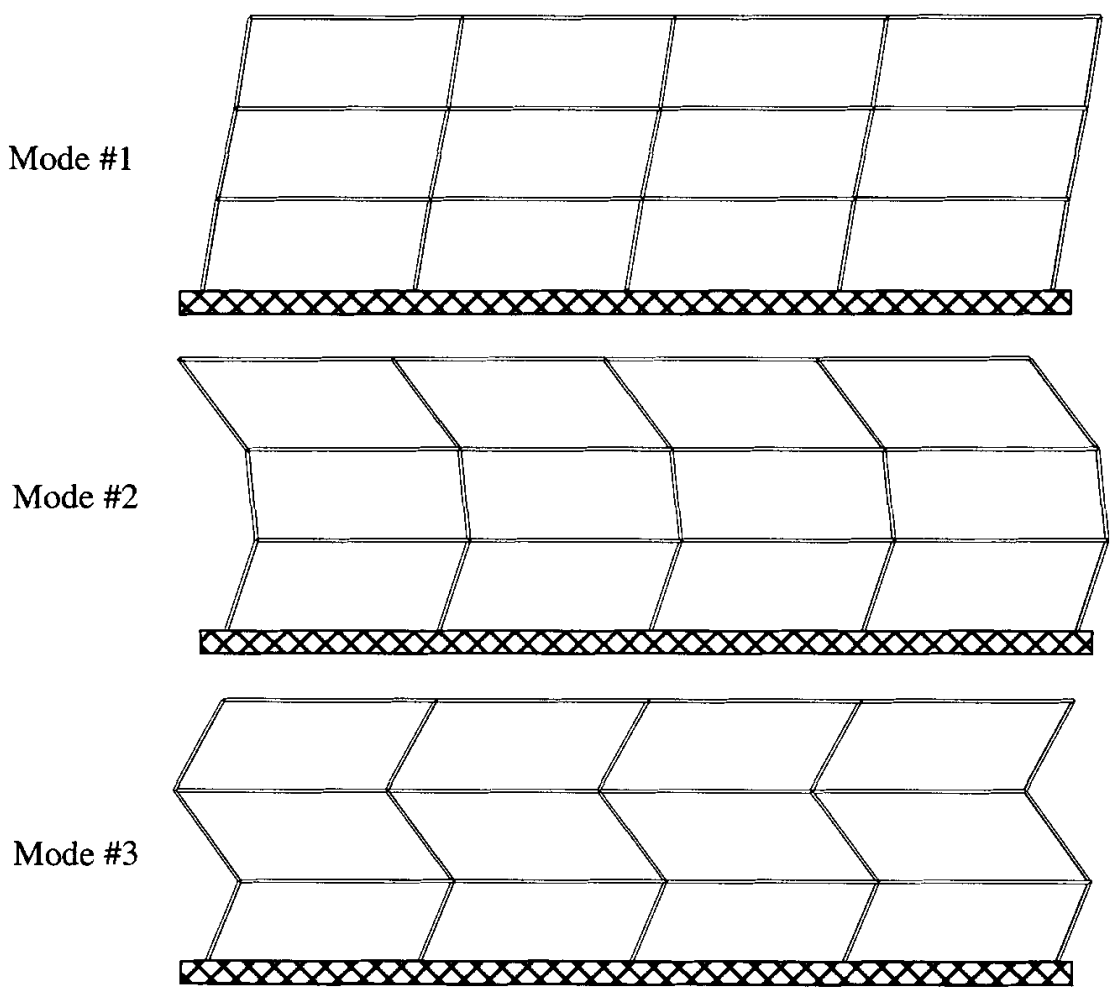

\begin{tabular}{|c|c|c|}
\hline Mode \# & $\begin{array}{c}\text { Frequency (Hz) } \\
\text { Period (sec) }\end{array}$ & $\begin{array}{c}\text { Frequency (Hz) } \\
\text { Gemini model }\end{array}$ \\
\hline 1 & $1.10 \mathrm{~Hz}(0.909 \mathrm{sec})$ & $1.10 \mathrm{~Hz}$ \\
\hline 2 & $3.49 \mathrm{~Hz}(0.287 \mathrm{sec})$ & $3.50 \mathrm{~Hz}$ \\
\hline 3 & $5.96 \mathrm{~Hz}(0.168 \mathrm{sec})$ & $5.96 \mathrm{~Hz}$ \\
\hline
\end{tabular}

FIGURE 41. Three story steel moment resisting frame. 


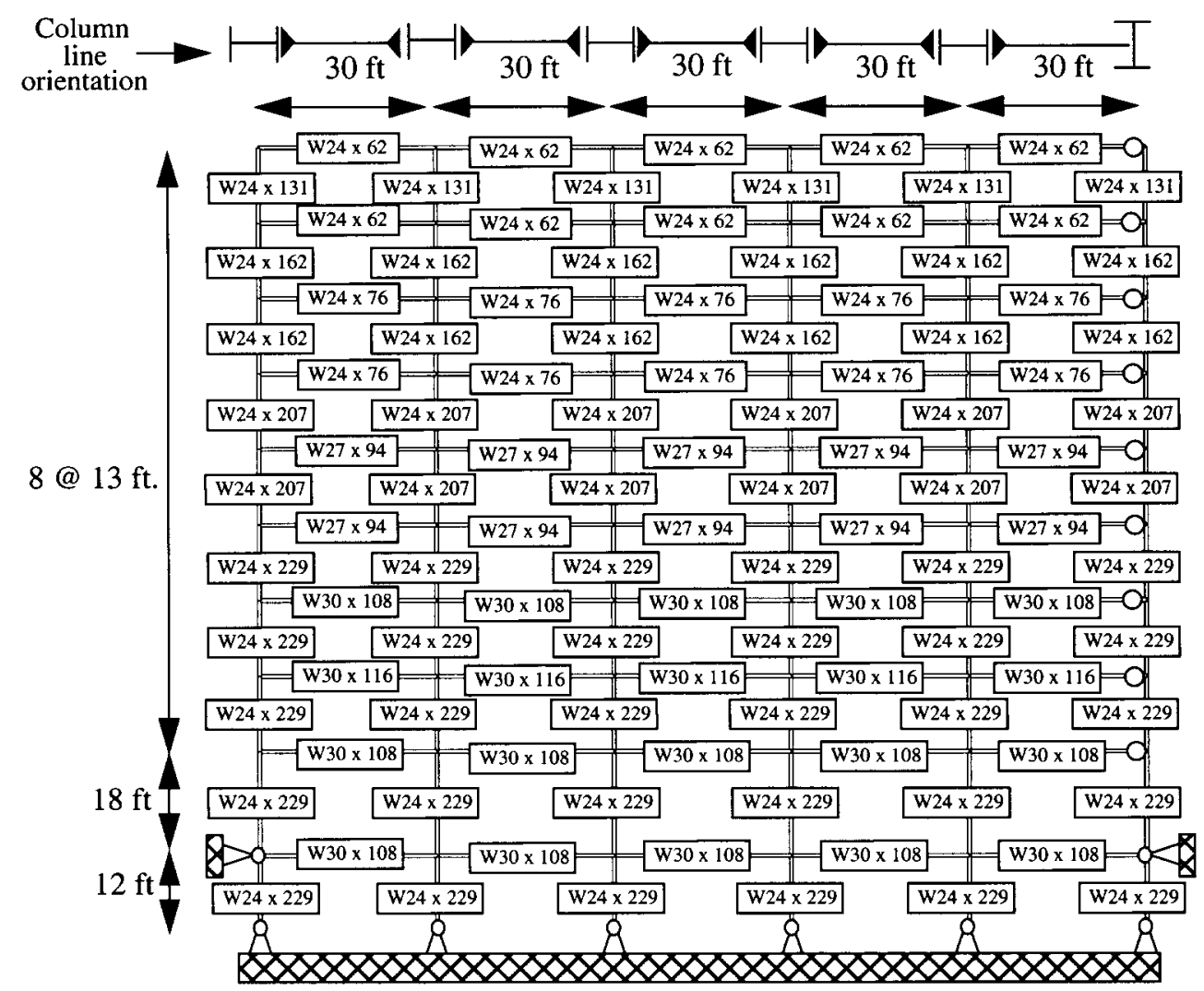

Total tributary weight to frame $=6.595 \times 10^{6} \mathrm{lb}$
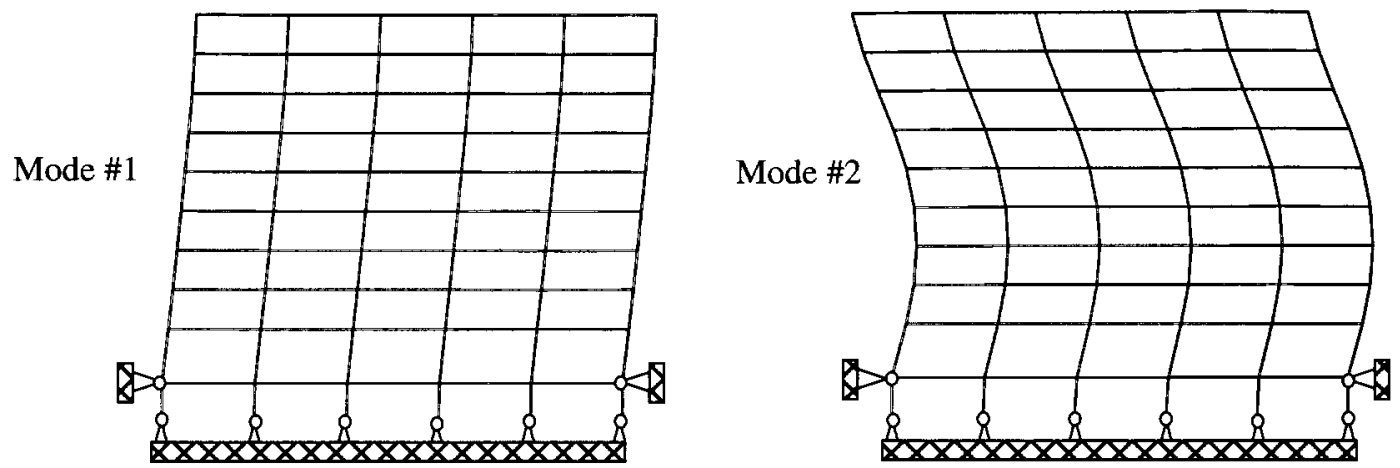

Mode \#3

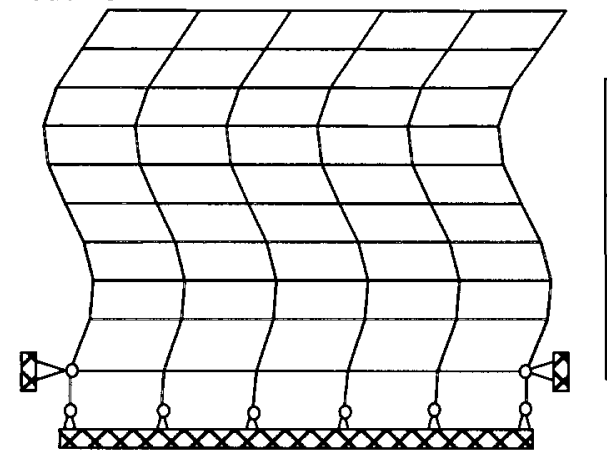

\begin{tabular}{|c|c|c|}
\hline Mode \# & $\begin{array}{c}\text { Frequency (Hz) } \\
\text { Period (sec) }\end{array}$ & $\begin{array}{c}\text { Frequency (Hz) } \\
\text { Gemini model }\end{array}$ \\
\hline 1 & $0.486 \mathrm{~Hz}(1.95 \mathrm{sec})$ & $0.486 \mathrm{~Hz}$ \\
\hline 2 & $1.26 \mathrm{~Hz}(0.763 \mathrm{sec})$ & $1.26 \mathrm{~Hz}$ \\
\hline 3 & $2.28 \mathrm{~Hz}(0.437 \mathrm{sec})$ & $2.28 \mathrm{~Hz}$ \\
\hline
\end{tabular}

FIGURE 42. Nine story steel moment resisting frame. 


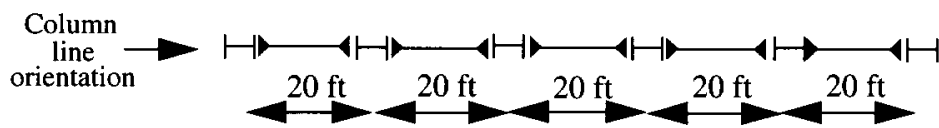

$19 @ 13 \mathrm{ft}$

Total tributary weight to frame $=8.525 \times 10^{6} \mathrm{lb}$

FIGURE 43. Twenty story steel moment resisting frame.

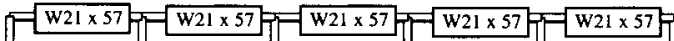

\begin{tabular}{|l|l|l|}
\hline $\mathrm{W}_{24} \times 131$ & $\mathrm{~W} 24 \times 131$ \\
\hline
\end{tabular}

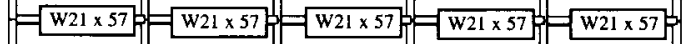

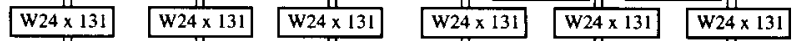

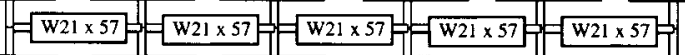

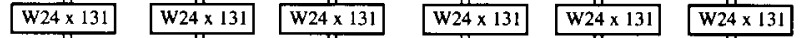
$\mid=\mathrm{W}_{24} \times 62=\mathrm{W}_{24} \times 62=\mathrm{W}_{24} \times 62 \mathrm{~W}=\mathrm{W}_{24} \times 62$

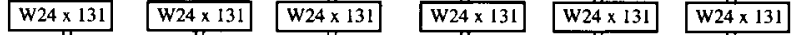

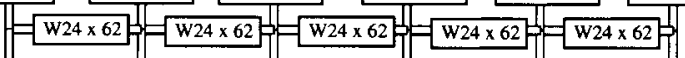

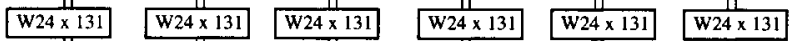
W27 × 94 $=$ W27 ×94

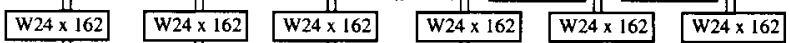
$=\mathrm{W}_{27 \times 94}=\mathrm{W}_{27 \times 94}=\mathrm{W}_{27 \times 94} \mathrm{P}_{=}=\mathrm{W} 27 \times 94=\mathrm{W} 27 \times 94$

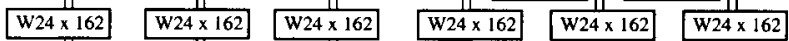
$=\mathrm{w}_{27 \times 94}=\mathrm{W} 27 \times 94=\mathrm{W} 27 \times 94=\mathrm{W} 27 \times 94=\mathrm{W} 27 \times 94$

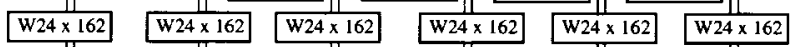

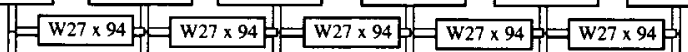

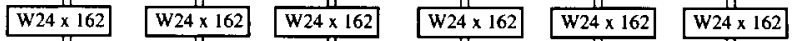
W27 ×114

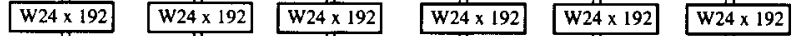
W27 × 114

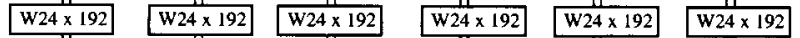

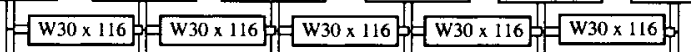

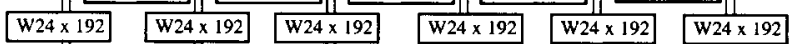
W= W30 116

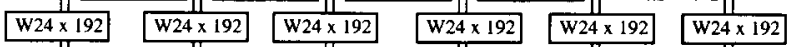

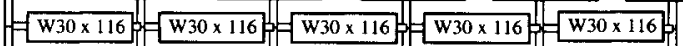

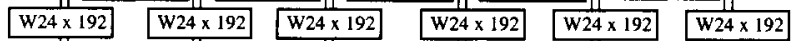

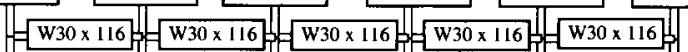

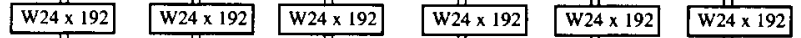
$=\mathrm{w} 30 \times 132=\mathrm{W} 30 \times 132=\mathrm{W} 30 \times 132, \mathrm{~W} 30 \times 132, \mathrm{~W} 30 \times 132$

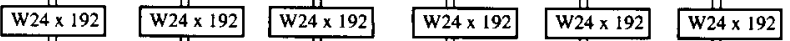

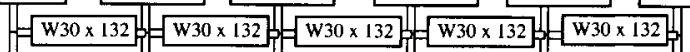

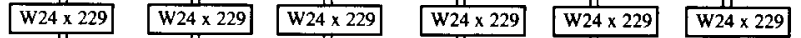

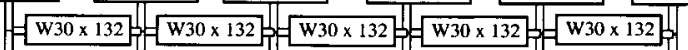

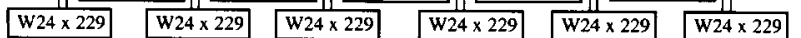

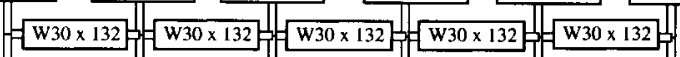

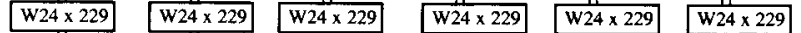

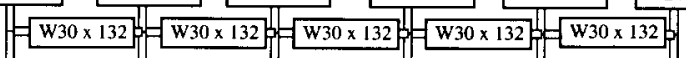

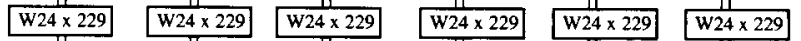

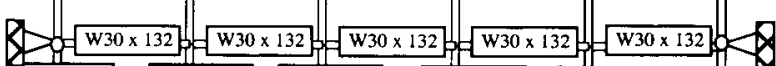

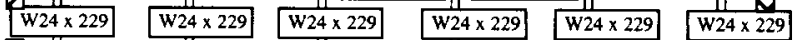
$\mathrm{W}_{\mathrm{O}}=\mathrm{W}_{12 \times 14}=\mathrm{W}_{12 \times 14}=\mathrm{W}_{12 \times 14}=\mathrm{W}_{12} \times 14=\mathrm{W}_{12} \times 14=8$

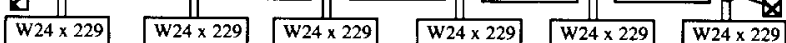
$12 \mathrm{ft}$ ס

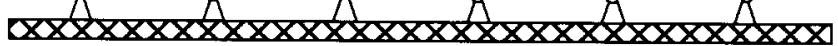



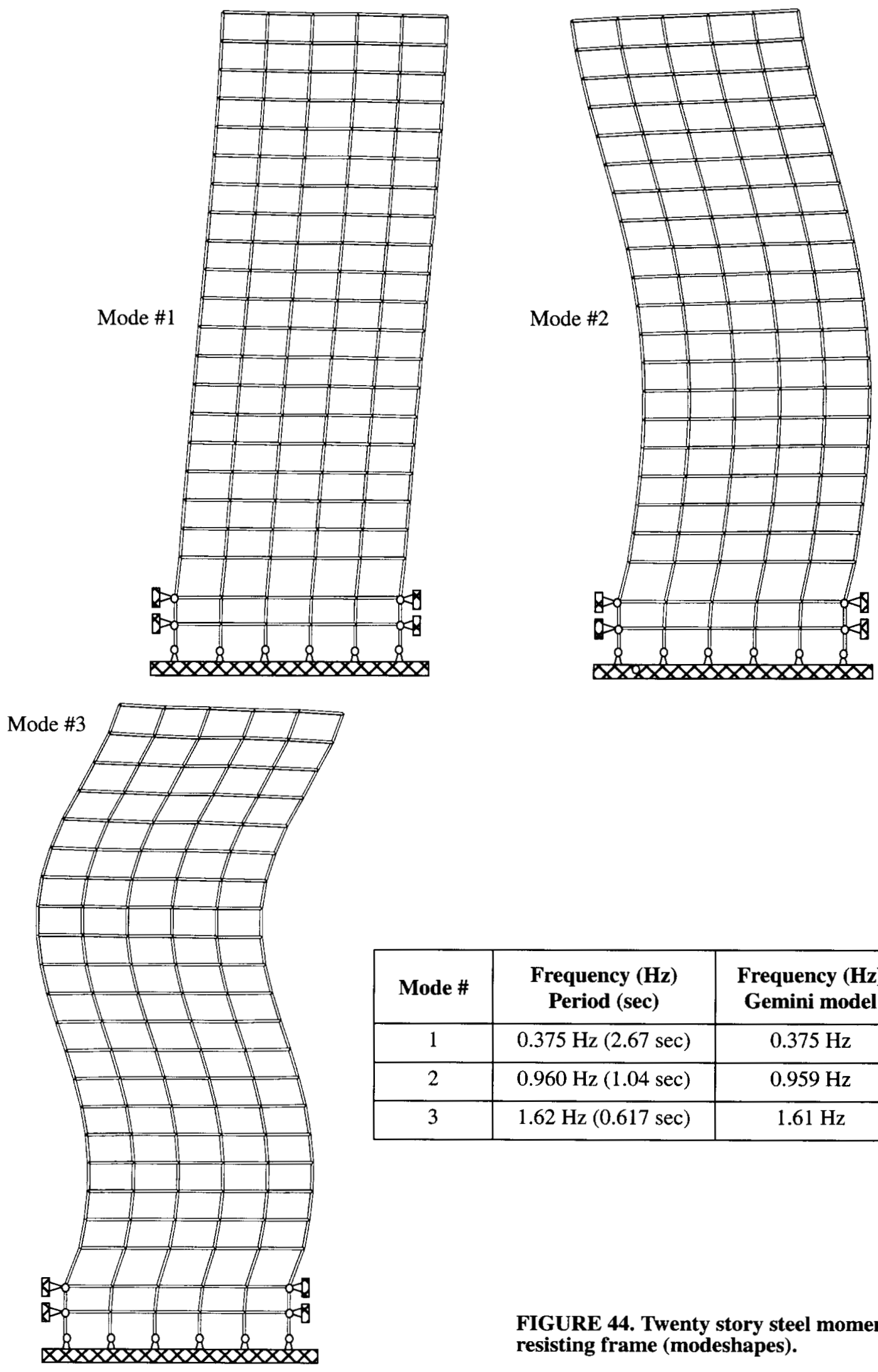

\begin{tabular}{|c|c|c|}
\hline Mode \# & $\begin{array}{c}\text { Frequency (Hz) } \\
\text { Period (sec) }\end{array}$ & $\begin{array}{c}\text { Frequency (Hz) } \\
\text { Gemini model }\end{array}$ \\
\hline 1 & $0.375 \mathrm{~Hz}(2.67 \mathrm{sec})$ & $0.375 \mathrm{~Hz}$ \\
\hline 2 & $0.960 \mathrm{~Hz}(1.04 \mathrm{sec})$ & $0.959 \mathrm{~Hz}$ \\
\hline 3 & $1.62 \mathrm{~Hz}(0.617 \mathrm{sec})$ & $1.61 \mathrm{~Hz}$ \\
\hline
\end{tabular}

FIGURE 44. Twenty story steel moment resisting frame (modeshapes). 


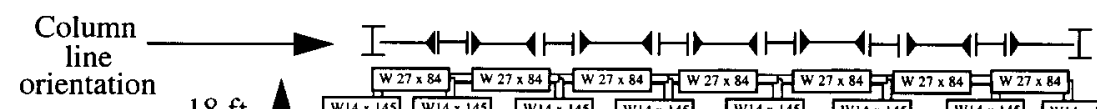

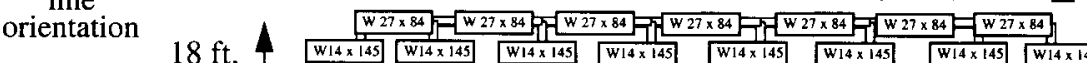

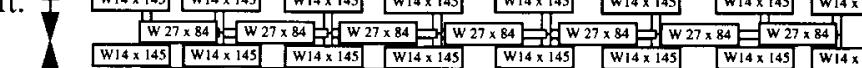

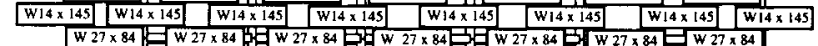

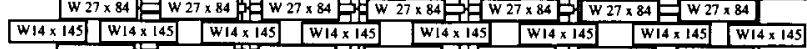

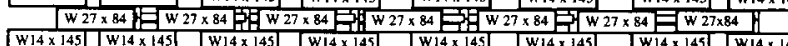

\begin{tabular}{l} 
W \\
\hline
\end{tabular} W 27×84

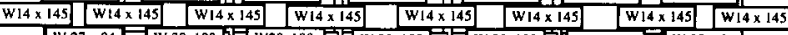

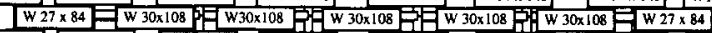

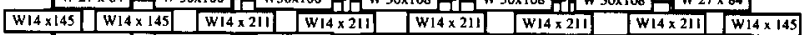

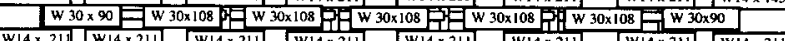

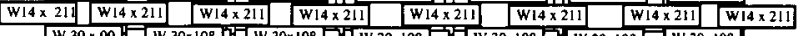

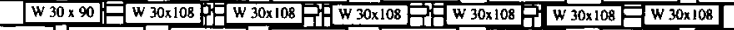

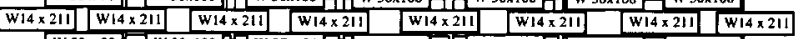

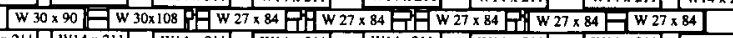

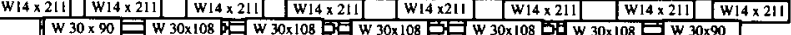

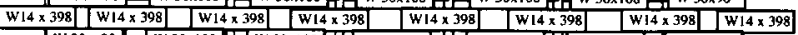

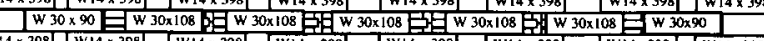

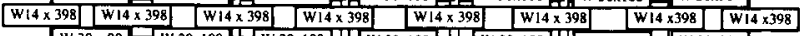

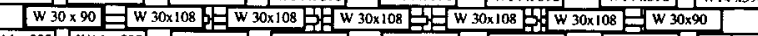

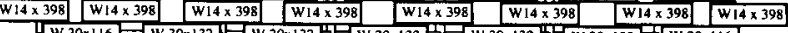

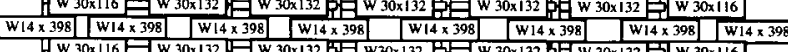
W

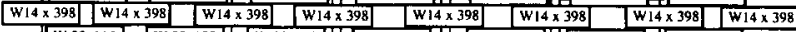

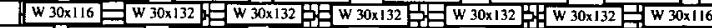

\begin{tabular}{|l|l|l|l|l|l|l|l|l|}
\hline$W_{14} \times 398$ & $W_{14} \times 398$ & $W_{14} \times 398$ & $W_{14} \times 398$ & $W_{14} \times 398$ & $W_{14} \times 398$ & $W_{14} \times 398$ & $W_{14} \times 398$ \\
\hline
\end{tabular}

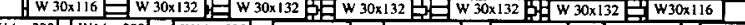

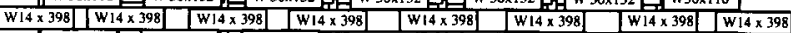

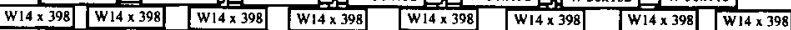

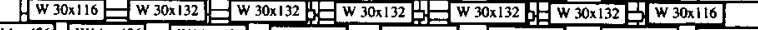

\begin{tabular}{|l|l|l|l|l|l|l|l|}
\hline$w_{14} \times 426$ & $w_{14} \times 426$ & $W_{14} \times 426$ & $w_{14} \times 426$ & $w_{14} \times 426$ & $w_{14} \times 426$ & $w_{14} \times 426$ & $w_{14} \times 426$ \\
\hline
\end{tabular}

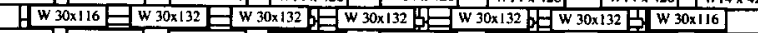

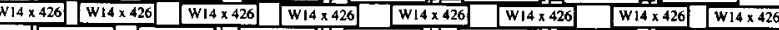

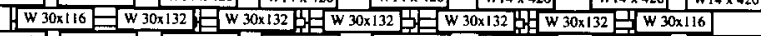

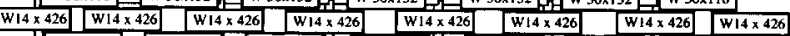

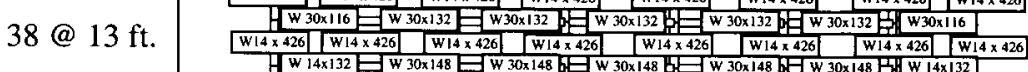

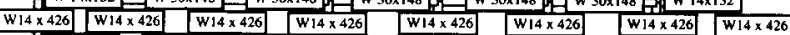

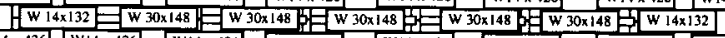

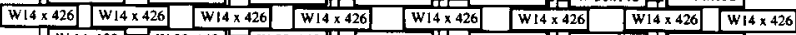

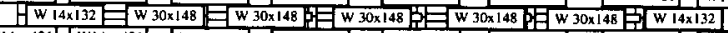

\begin{tabular}{|l|l|l|l|l|l|l|l|l|l|l|}
\hline$w_{14} \times 426$ & $w_{14} \times 426$ & $w_{14} \times 426$ & $w_{14} \times 426$ & $\mid w_{14} \times 426$ & $w_{14} \times 426$ & $w_{14} \times 426$ & $w_{14} \times 426$ \\
\hline
\end{tabular}

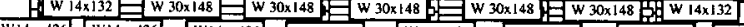

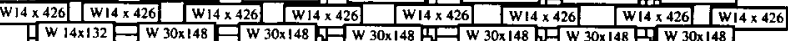

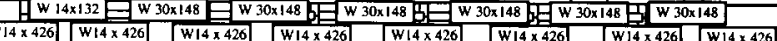

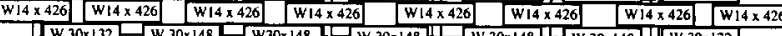

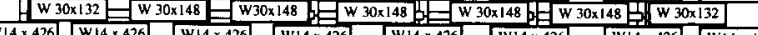

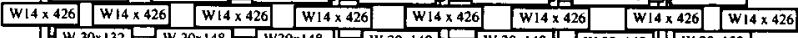

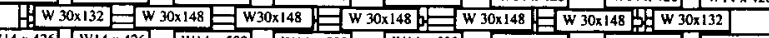

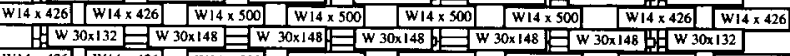

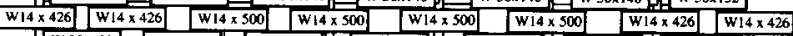

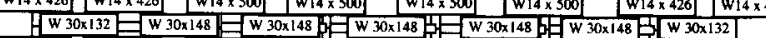

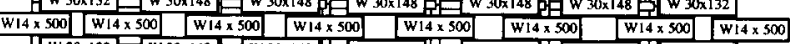

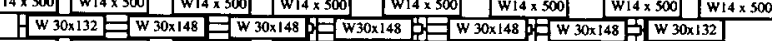

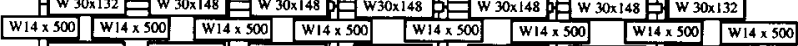

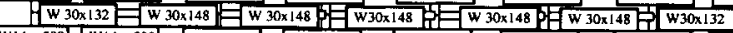

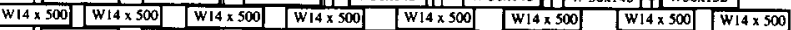

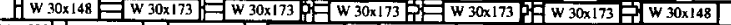

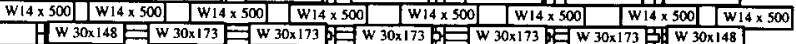

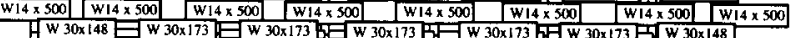

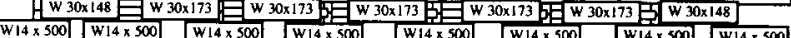

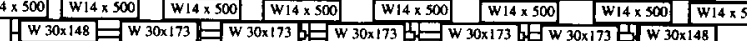

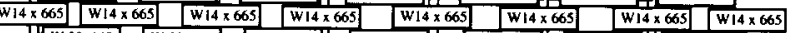

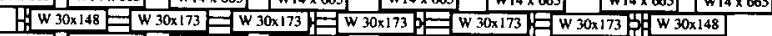

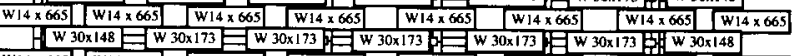

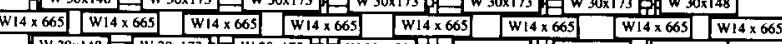

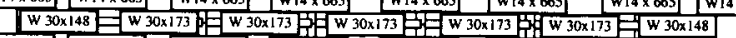

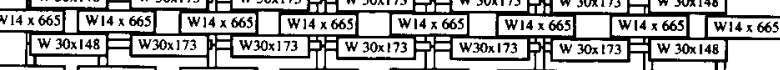

RE 45. Forty story steel moment resisting frame.

$18 \mathrm{ft}$.

$3 @ 14 \mathrm{ft}$.

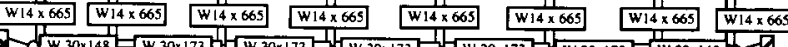

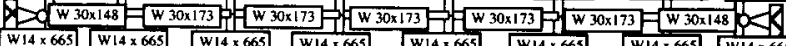

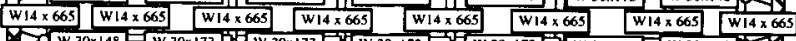

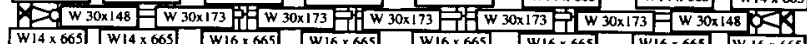

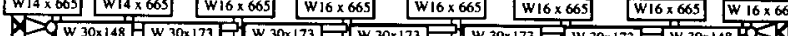

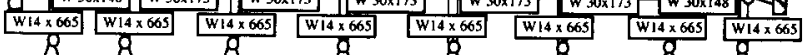

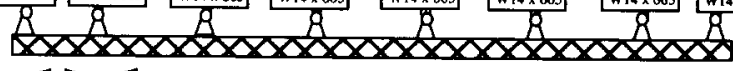

$20 \mathrm{ft}$.

5 @ $30 \mathrm{ft}$.

$20 \mathrm{ft}$. 

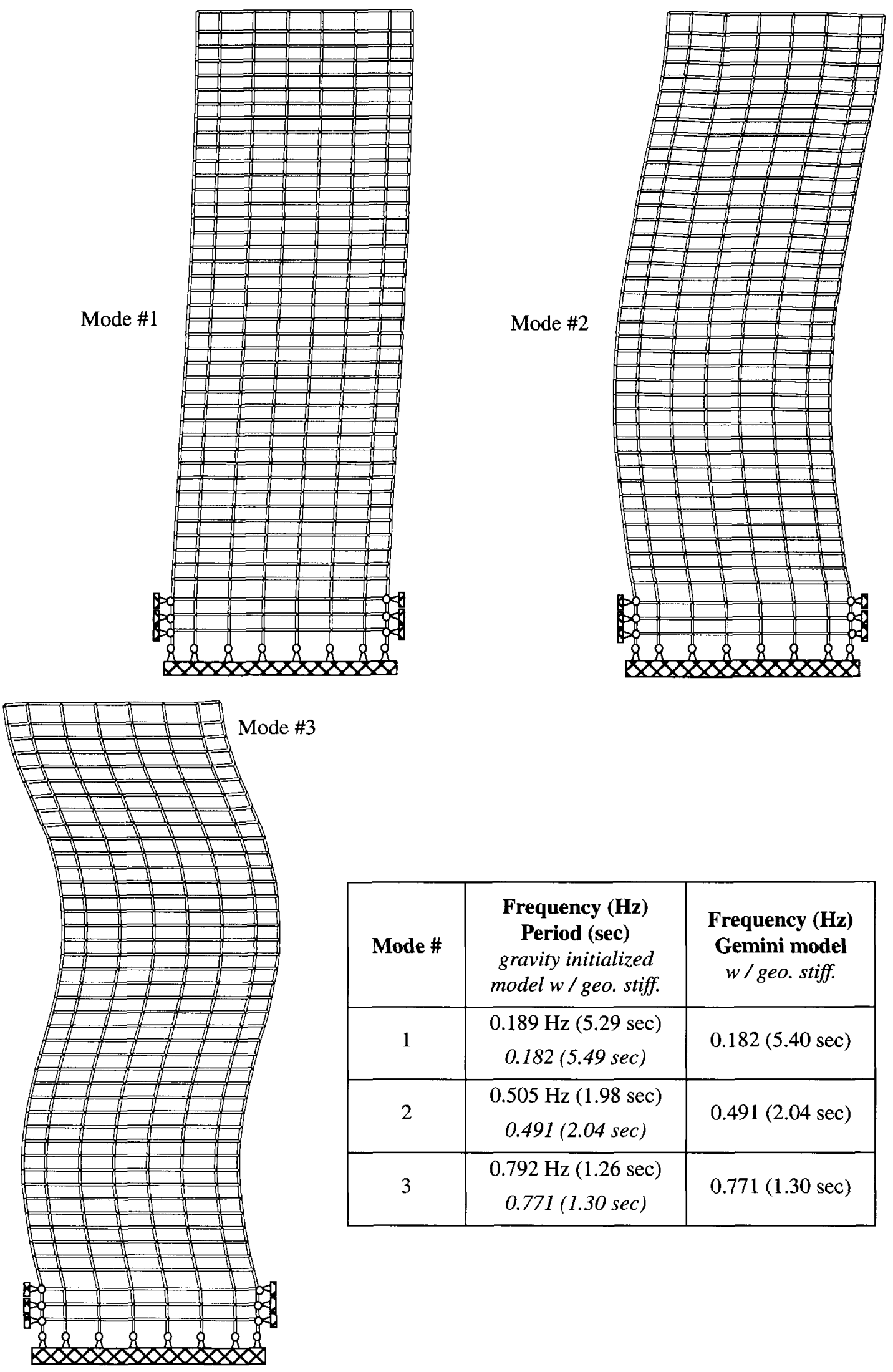

\begin{tabular}{|c|c|c|}
\hline Mode \# & $\begin{array}{c}\text { Frequency (Hz) } \\
\text { Period (sec) } \\
\text { gravity initialized } \\
\text { model } w / \text { geo. stiff. }\end{array}$ & $\begin{array}{c}\text { Frequency }(\mathbf{H z}) \\
\text { Gemini model } \\
\text { w/geo. stiff. }\end{array}$ \\
\hline 1 & $\begin{array}{c}0.189 \mathrm{~Hz}(5.29 \mathrm{sec}) \\
0.182(5.49 \mathrm{sec})\end{array}$ & $0.182(5.40 \mathrm{sec})$ \\
\hline 2 & $\begin{array}{c}0.505 \mathrm{~Hz}(1.98 \mathrm{sec}) \\
0.491(2.04 \mathrm{sec})\end{array}$ & $0.491(2.04 \mathrm{sec})$ \\
\hline 3 & $0.792 \mathrm{~Hz}(1.26 \mathrm{sec})$ & $0.771(1.30 \mathrm{sec})$ \\
& $0.771(1.30 \mathrm{sec})$ & \\
\hline
\end{tabular}


Axial displacement of an elasto-plastic beam

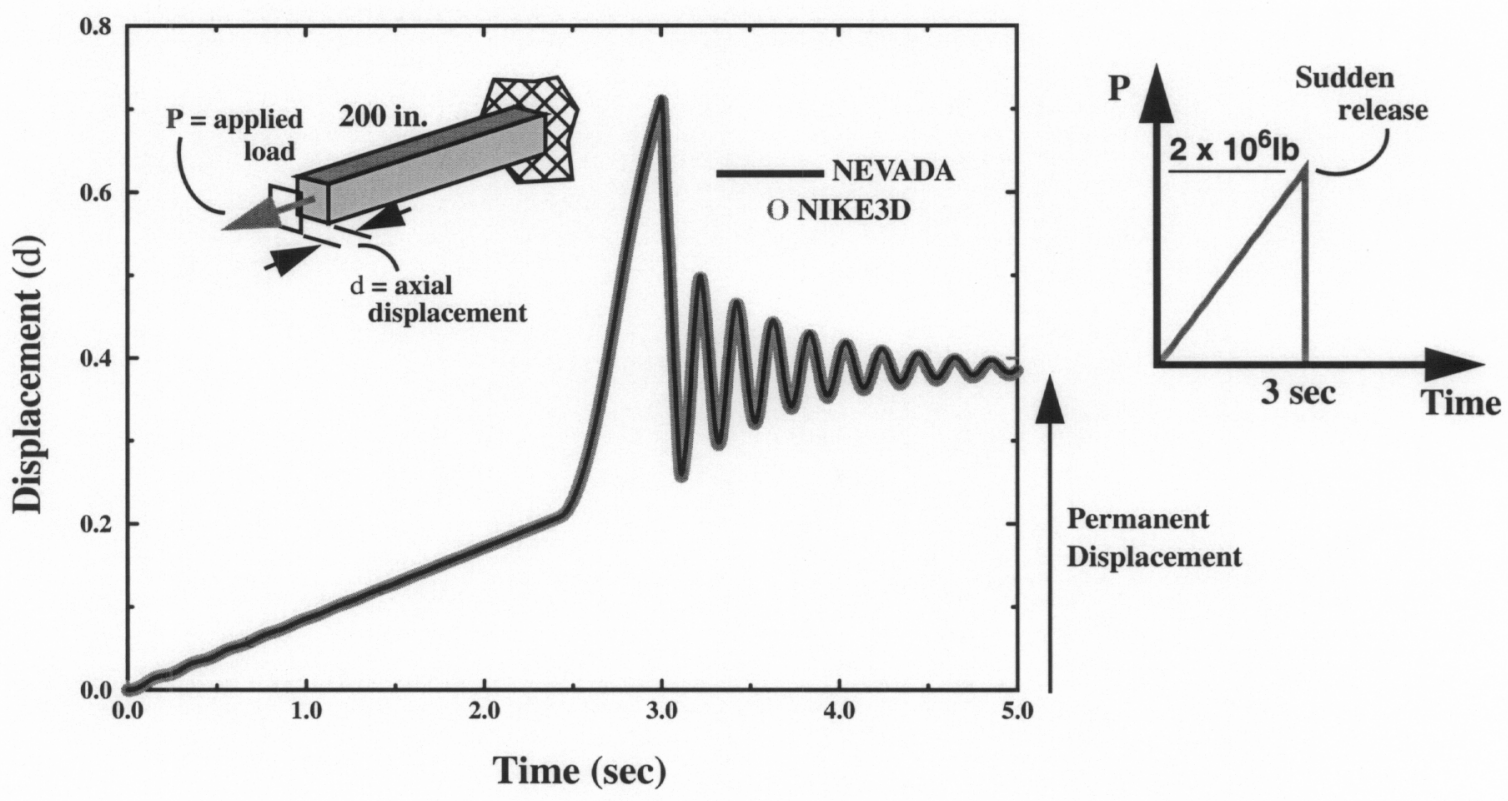

FIGURE 46. Elasto-plastic deformation of a wide-flange beam (W14x176).

Cantilever displacement of an elasto-plastic beam

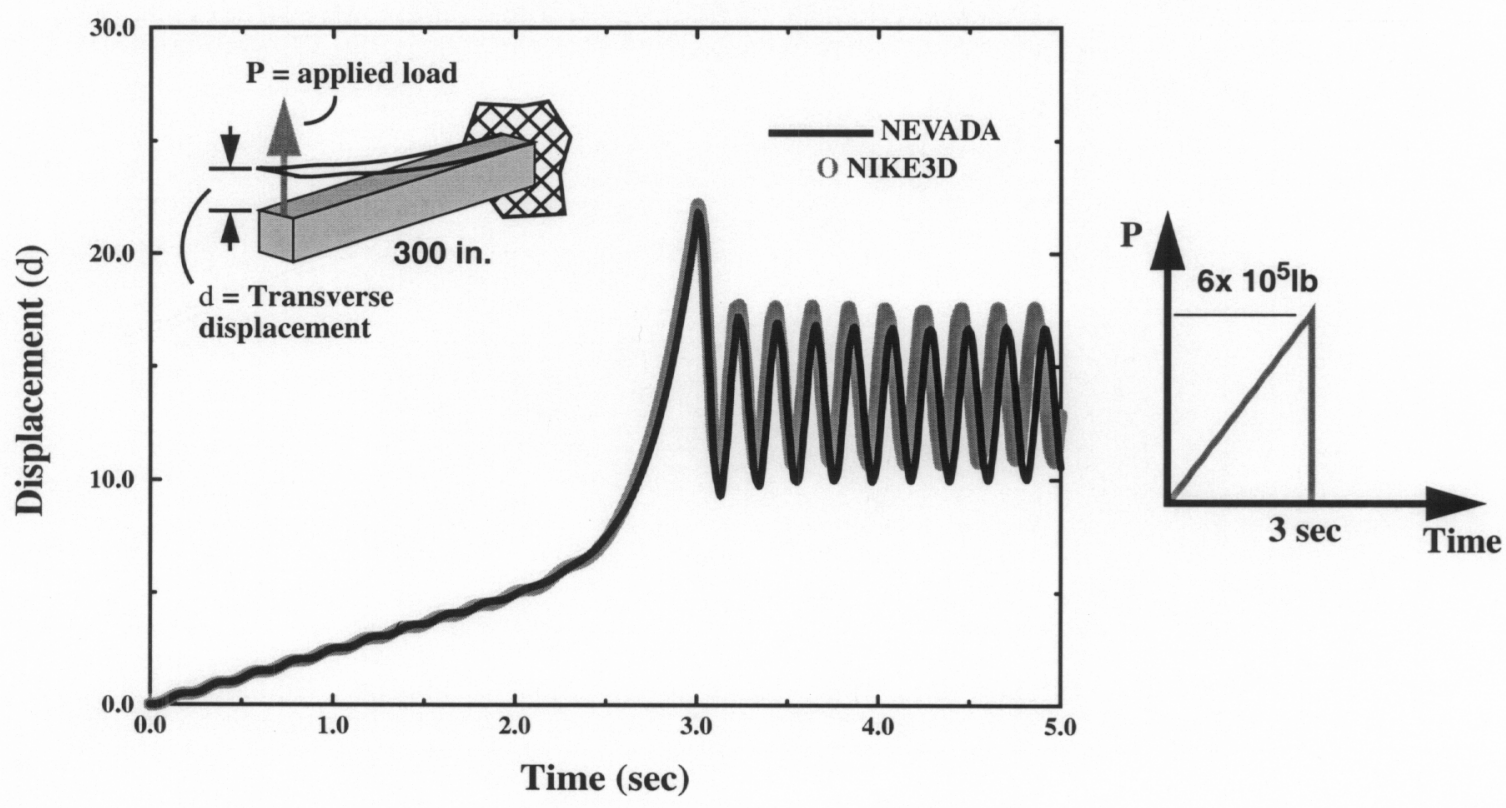

FIGURE 47. Elasto-plastic deformation of a wide-flange beam (W14x176). 


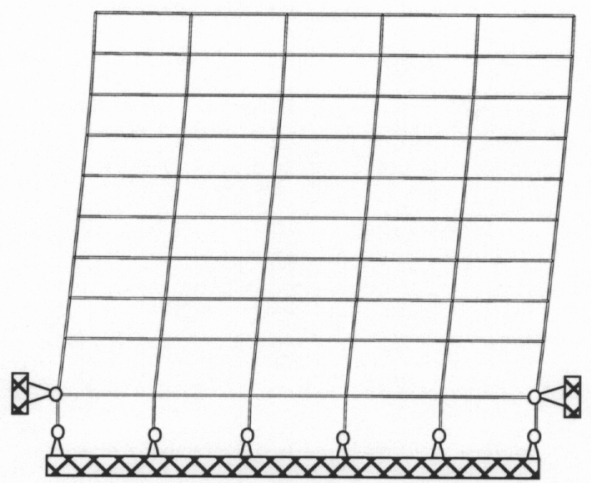

a)

Landers Lucerne station from Iwan et. al.
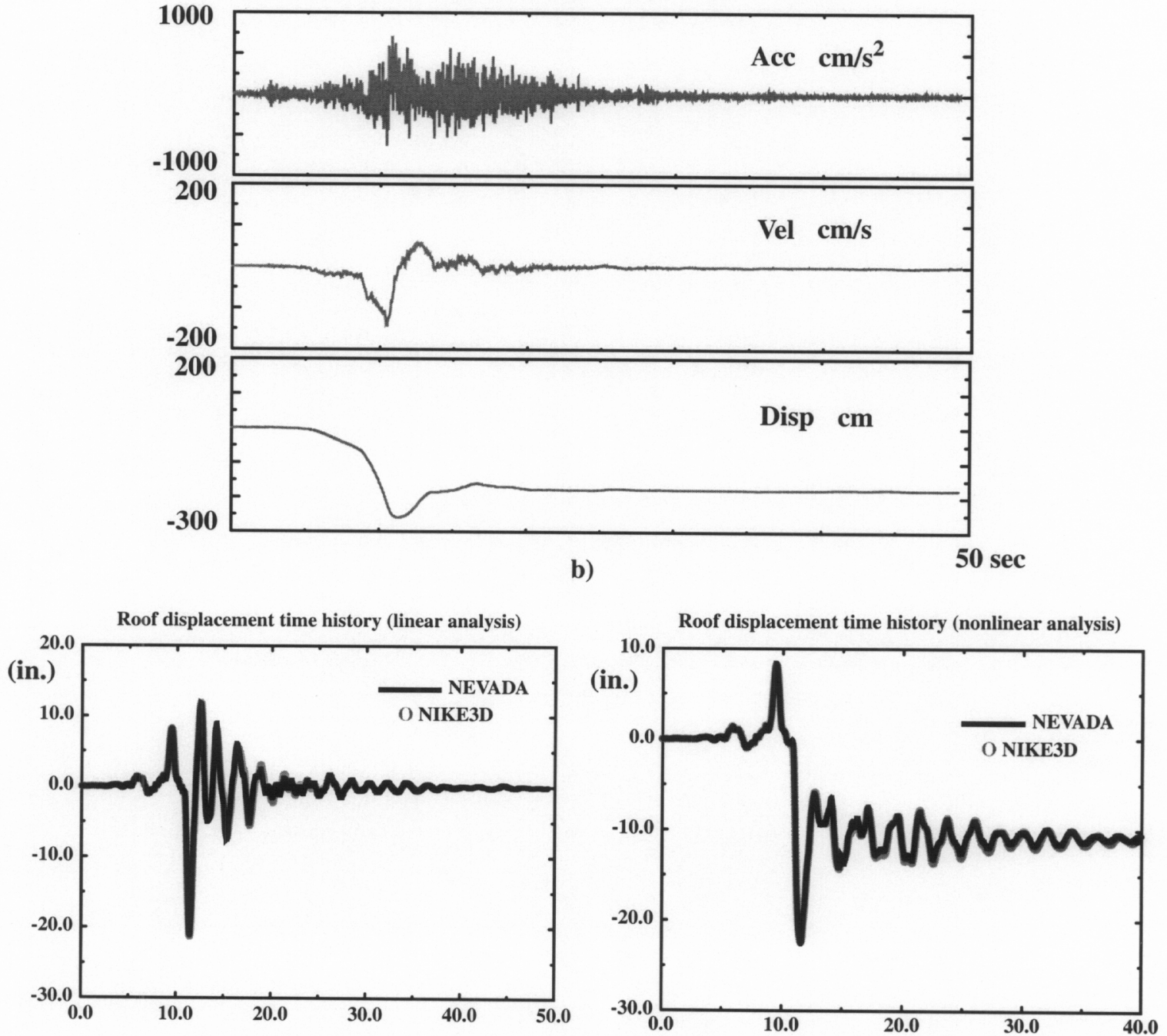

c)

FIGURE 48. Linear and nonlinear response of a nine story frame subjected to near-field earthquake records. a) Nine story steel moment resisting frame; b) earthquake ground motion acceleration time histories; c) computed response for linear and nonlinear analyses. 